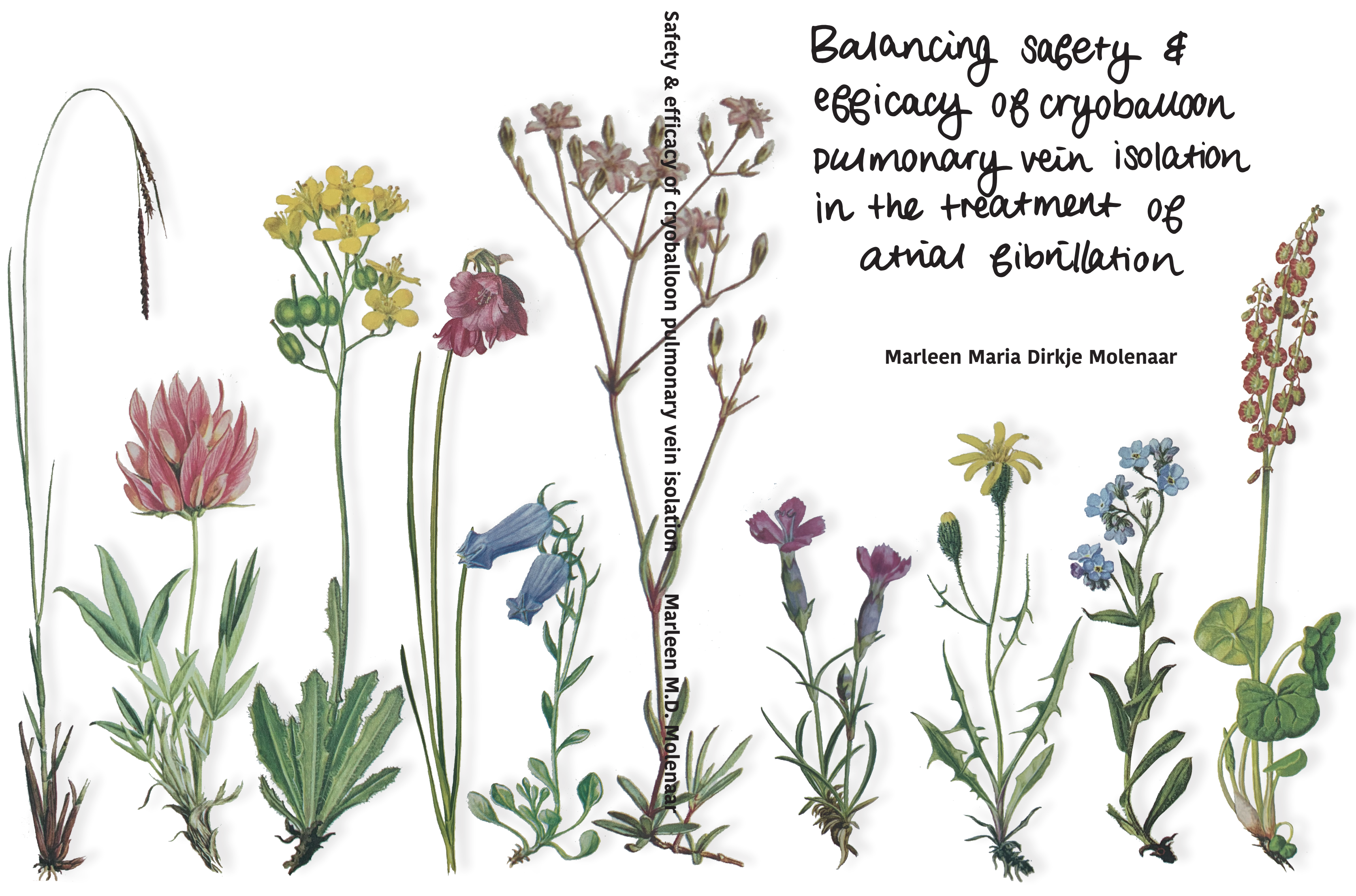




\section{BALANCING SAFETY AND EFFICACY OF CRYOBALLOON PULMONARY VEIN ISOLATION IN THE TREATMENT OF ATRIAL FIBRILLATION}

Marleen Maria Dirkje Molenaar 


\title{
BALANCING SAFETY AND EFFICACY OF CRYOBALLOON PULMONARY VEIN ISOLATION IN THE TREATMENT OF ATRIAL FIBRILLATION
}

\section{PROEFSCHRIFT}

\author{
ter verkrijging van \\ de graad van doctor aan de Universiteit Twente \\ op gezag van de rector magnificus, \\ prof. dr. T.T.M. Palstra, \\ volgens besluit van het College voor Promoties \\ in het openbaar te verdedigen \\ op vrijdag 19 juni 2020 om 14:45
}

door

\section{Marleen Maria Dirkje Molenaar}

geboren op 15 februari 1987

te Purmerend, Nederland 
Dit proefschrift is goedgekeurd door de promotoren:

Prof. dr. J.G. Grandjean

Prof. dr. ir. B. ten Haken

De co-promotor:

Dr. J.M. van Opstal

(C2020 Marleen M.D. Molenaar, The Netherlands

Cover design: Liza Hidding \& Marleen Molenaar

Printed by: Ipskamp printing

ISBN: 978-90-365-4986-8

DOI: $10.3990 / 1.9789036549868$

Cover drawings based on: Elseviers alpengids

Printing of this thesis was kindly supported by Stichting Hartcentrum Twente, Medical School Twente and Magnetic Detection \& Imaging group.

All rights reserved. No parts of this thesis may be reproduced, stored in a retrieval system or transmitted in any form or by any means without permission of the author. 


\section{Promotiecommissie}

Voorzitter \& secretaris

Promotoren

Co-promotor

Leden
Prof. dr. J.L. Herek

Prof. dr. J.G. Grandjean

Prof. dr. ir. B. ten Haken

Dr. J.M. van Opstal

Prof. dr. J.A.M. van der Palen

Prof. dr. C. von Birgelen

Prof. dr. N.M.S. de Groot

Prof. dr. H.J.G.M Crijns

Dr. M.F. Scholten 


\section{Table of Contents}

Chapter 1 General introduction and outline of the thesis

Chapter 2 Safety (first) in cryoballoon and radiofrequency pulmonary vein isolation for atrial fibrillation

Chapter 3 Shorter cryoballoon applications times do affect efficacy but result in less phrenic nerve injury: Results of the randomized 123 study Pacing and Clinical Electrophysiology 2019

Chapter 4 Shorter right superior pulmonary vein cryoapplications result in 73 less phrenic nerve injury and similar 1-year freedom from atrial fibrillation Accepted (with revisions) for publication in Pacing and Clinical Electrophysiology

Chapter 5 High incidence of (ultra)low oesophageal temperatures during 89 cryoballoon pulmonary vein isolation for atrial fibrillation Accepted (with revisions) for publication in Netherlands Heart Journal

Chapter 6 Use of three-dimensional computed tomography overlay for 109 real-time cryoballoon ablation in atrial fibrillation reduces radiation dose and contrast dye Netherlands Heart Journal. 2017

Chapter 7 Prevalence and consequences of incidental findings detected by 127 computed tomography prior to pulmonary vein isolation or transcatheter aortic valve replacement

Chapter 8 Summary, conclusion and future perspectives 145

Chapter 9 Nederlandse samenvatting, conclusie en toekomstperspectieven 161

Chapter 10 Portfolio, Dankwoord \& Curriculum Vitae 177 
CHAPTER 1

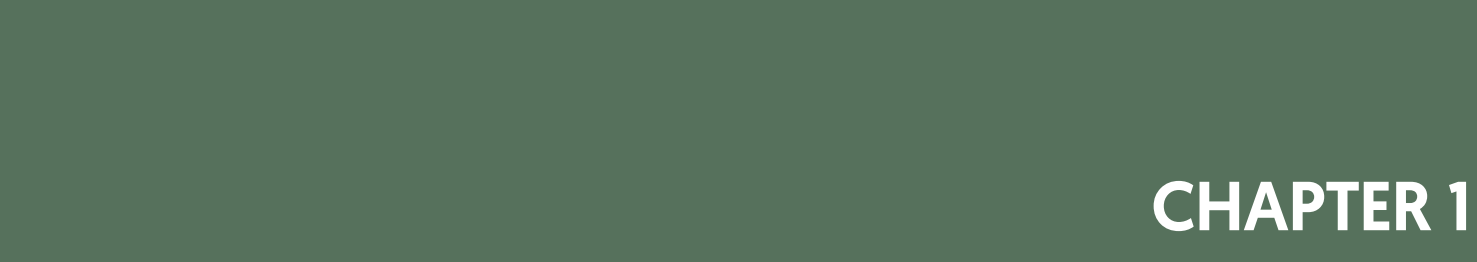




\section{GENERAL INTRODUCTION AND OUTLINE OF THE THESIS}

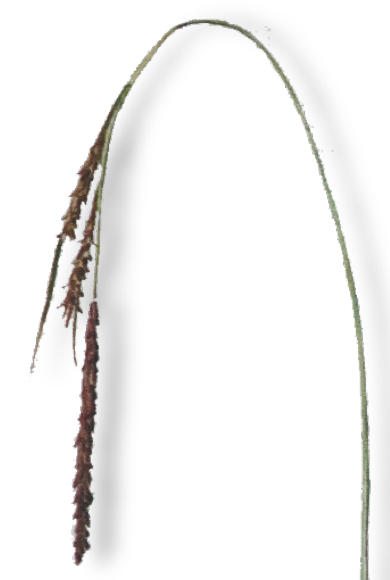




\section{General introduction}

\section{Atrial fibrillation}

Atrial fibrillation (AF) is an atrial arrhythmia characterized by irregular heart rate due to diffuse and chaotic atrial activity and irregular ventricular response. With a prevalence ranging from $2-3 \%$ in the EU and the USA it is not only the most common sustained cardiac arrhythmia but also one of the most important public health issues'. The prevalence of AF is expected to double over the next 50 years as the population ages ${ }^{2}$.

The prevalence of AF increases with advanced age, the reported prevalence for the population below 49 years of age is between 0.1 and $0.2 \%$ and over $10 \%$ in people older than 75 years $^{2}$. AF is more common in men than women, with an approximated ratio of 1.2:1. Patients with conditions as hypertension, heart failure, coronary artery disease, valvular heart disease, obesity, diabetes mellitus, or chronic kidney disease also have a higher risk for $\mathrm{AF}^{3-9}$.

Symptoms of AF vary widely among patients. In a discrete $10-15 \%$ of the patients, particularly in elderly, AF occurs in the absence of symptoms, therefore the actual prevalence of AF is probably still underestimated ${ }^{10-12}$. The most common symptom of AF is fatigue but patients also commonly present themselves with palpitations, shortness of breath, exercise intolerance and chest pain.

The main risk of AF is the occurrence of thromboembolic events. AF is associated with a 5 -fold increase in risk of stroke resulting in a significant morbidity and mortality 6 . AF is also associated with a 3 fold risk of heart failure while tachycardiainduced cardiomyopathy may develop when the ventricular rate is not adequately controlled ${ }^{13}$. Both the risk of thromboembolic events and the ventricular rate can be well controlled by pharmacological treatment. Therefore AF is not a lifethreatening arrhythmia. However, it can have a significant effect on the quality of life.

Typically AF starts with a paroxysmal pattern, coming in episodes starting and terminating spontaneously, without intervention, in less than 7 days. In time AF progresses to a permanent form in the majority of patients where AF is continuous, and interventions to restore sinus rhythm have either failed or not been attempted. The progressive nature of was found to result from electrical and structural remodelling known as "AF begets AF" 
Both a trigger, a focal electrical activation, as well as an atrial substrate capable to promote and maintain the electrical activation, are required for AF to be induced. In paroxysmal AF the left atrial myocardial sleeves represent the most common origin of focal electrical activation ${ }^{15}$. Around the pulmonary veins, the myocardial sleeves extend beyond the cardiac silhouette creating electrophysiological characteristics favourable for spontaneous electrical activation ${ }^{16}$. On the ECG AF can be characterized by an irregular ventricular interval and the absence of distinct organized atrial activity.

\section{Pulmonary vein isolation}

Rhythm control of AF by drug therapy has shown limited efficacy and is often associated with side effects. After it was discovered that AF is triggered from the pulmonary veins (PVs) in the majority of cases, electrical isolation of the PVs by ablation has become the cornerstone in symptomatic AF treatment ${ }^{15}$. Catheter ablation has proven to be superior to anti-arrhythmic drugs in rhythm control ${ }^{17,18}$.

The historical approach to achieve complete PV isolation (PVI) is point-by-point catheter ablation using radio frequency (RF) causing cellular necrosis by heat. RF catheter ablation for $\mathrm{PVI}$ is a technically challenging and time consuming procedure. Therefore alternatives to point-by-point RF PVI have been introduced over recent years. These alternatives, such as cryoballoon, laser balloon and multielectrode phased array RF catheters, aim to reduce PVI complexity ${ }^{19-21}$.

\section{Cryoballoon PVI}

The cryoballoon was first introduced in 2006. The technique is based on thermal lesion creation by extremely low temperatures. The cryoballoon is positioned at the ostium of the PV where the balloon is inflated. The refrigerant, liquid nitrous oxide, is injected into the balloon through dedicated holes. There the refrigerant undergoes a phase transition from liquid to gas according to Boyle's law. The phase transition process consumes energy and therefore the temperature decreases, known as the Joule-Thomson effect, resulting in cooling at the surface of the balloon ${ }^{22}$. The temperature at the balloon surface can decrease down to approximately $-80^{\circ} \mathrm{C}$, creating thermal lesions at the site of balloon-to-tissue contact ${ }^{23}$. Cellular injury is caused by intra- and extracellular ice formation as well as microvascular injury ${ }^{24,25}$. Disappearance of electrical connection between the PV and the atrium can be observed during the procedure using a spiral mapping catheter. The catheter is inserted through an inner lumen within the balloon catheter and positioned in the PV. 
To achieve complete PVI the created lesions should be transmural and continuous. Adequate cooling energy delivery and complete occlusion are of key importance to achieve this. After promising results of the first-generation cryoballoon some technical modifications have been made (Figure 1). In the second-generation balloon the number of refrigerant injection ports was increased from four to eight, creating a broader and more homogeneous freezing zone. The third generation was equipped with a reduced catheter tip length. Due to the shorter tip, the spiral mapping catheter can be positioned closer to the PV ostium, providing a better recording of the PV potentials during the cryoapplication. Because of technical issues, the third-generation cryoballoon was withdrawn from the market shortly after its release. Recently it has been replaced with an equivalent in which these technical issues were solved, the fourth generation cryoballoon.

\section{Efficacy cryoballoon PVI}

In the early years of the cryoballoon, the main focus was on its efficacy and whether it could reach outcomes comparable to RF. One year freedom from atrial tachyarrhythmias for the first-generation cryoballoon and non-contact forcesensing RF catheters were both around 60\%. With the advent of the improved second-generation cryoballoon and contact-sensing RF catheters, one-year freedom from arrhythmia increased to around $80 \%$ for both methods ${ }^{26}$. Ultimately randomized controlled trials have shown that cryoballoon $\mathrm{PVI}$ is non-inferior to the classic point-by-point RF PVI in patients with paroxysmal $A F^{21,27,28}$.

The current guidelines indicate that RF and cryoballoon are the designated methods to perform PVI29. Recent data even suggest cryoballoon PVI is associated with a better outcome in clinical endpoints, such as freedom from AF and rate of major complications, when compared to $\mathrm{RF} \mathrm{PVI}^{30}$. In addition to its non-inferiority to RF in terms of safety and efficacy, the cryoballoon has a steeper learning curve and leads to fast and reproducible procedures ${ }^{21}$. Cryoballoon has even been suggested to be the most advantageous choice in first-time PVI1 ${ }^{31}$.

\section{Safety cryoballoon PVI}

However, the higher efficacy introduced by the second-generation cryoballoon goes hand in hand with an increased risk of complications. With more powerful cooling of the balloon, the energy spreads beyond the heart to critical structures as the lungs, oesophagus, phrenic and vagal nerves. The distribution of energy in surrounding tissues can be very extensive. Complications attributed to collateral damage are oesophageal ulcera and fistula, gastroparesis, phrenic nerve palsy and 


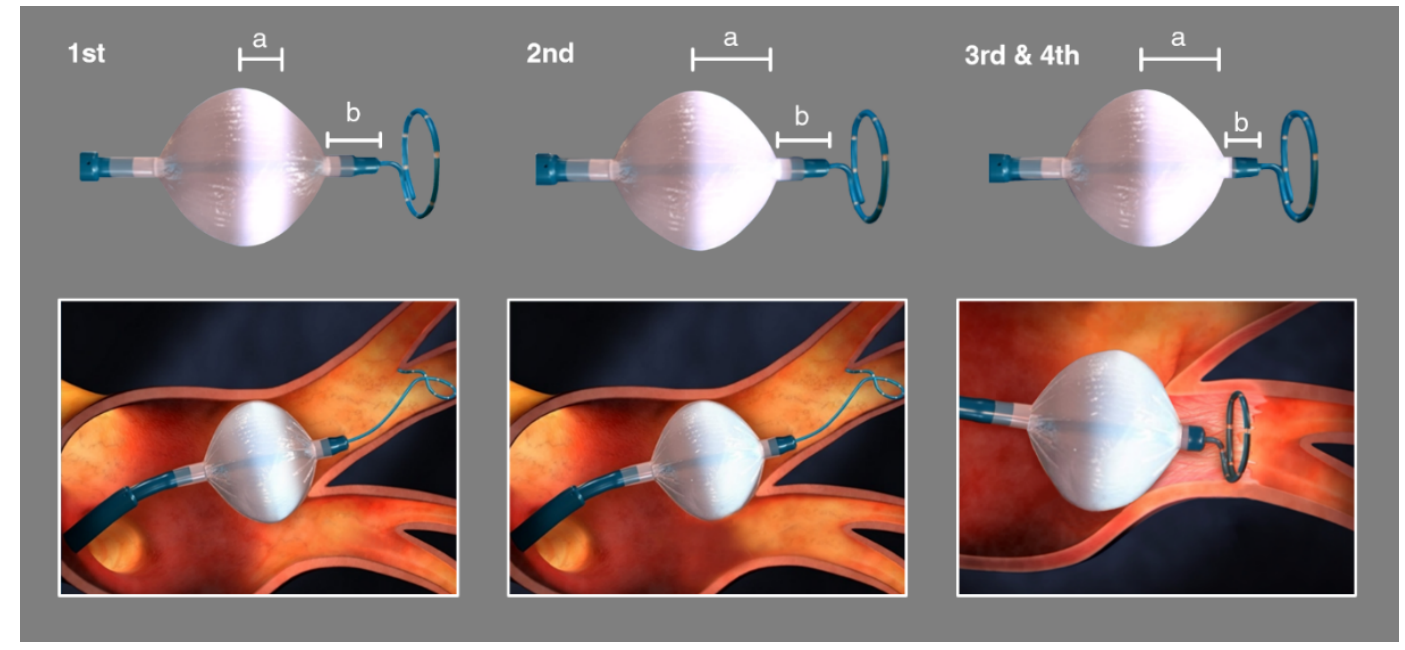

Figure 1 Top panel: first, second, third and fourth generation cryoballoon in which the cooling area was enlarged (a) and the tip was shortened (b). Bottom panel: cryoballoon situated at the ostium of the left superior pulmonary vein (1st and 2nd) and the left inferior pulmonary vein. The increased cooling surface ensures lesion creation around the complete ostium, also in anatomies in which aligning the balloon coaxially with the PV is challenging (1st vs 2nd). The shortened tip improves the ability to measure time-to-isolation by bringing the electrodes on the lasso closer to the muscular sleeve (3rd). (Adapted from figures provided by Medtronic Inc.) 
pulmonary complications. Compared to the first-generation, the second-generation cryoballoon is associated with a higher incidence of these complications ${ }^{32-35}$.

Over the recent years, the research paradigm shifted from efficacy to safety. With the aim to reduce extracardiac damage without affecting treatment efficacy, the dosing of cryoenergy became critically important. Historically, 4-minutes applications with a bonus application after isolation was the standard for the firstgeneration cryoballoon $^{36}$. The use of single and shorter applications as well as omitting bonus applications after successful isolation have been explored ${ }^{37-44}$. Protocols with shorter ablation times have shown encouraging results $s^{37,38,42}$. However, this has mainly been demonstrated retrospectively and the optimal cryoballoon duration and dosing strategy is still matter of debate.

Dosing is an important method to control the safety efficacy balance. Monitoring of critical structures as well as predicting imminent complications or patients at risk are additional areas for improvement. They would enable operators to take precautions in a specific subset of patients according to their particular risk factors and to prematurely cease applications if monitoring indicates imminent complications.

This thesis describes the search for an optimization of the balance between safety and efficacy in (cryoballoon) pulmonary vein isolation. As AF is a non-lifethreatening condition in itself, the treatment should always obey the "primum non nocere" statement, first do no harm. This thesis focusses on improvement of this safety aspect. 


\section{Outline of the thesis}

The general introduction in Chapter 1 describes the nature of the disease, AF, the rationale of PVI and the cryoballoon as a method to perform PVI as well as the current status and challenges considering its safety and efficacy, all to put the chapters of this thesis into context.

Chapter 2 elaborates on the safety aspect of as well cryoballoon as radiofrequency $\mathrm{PVI}$ and discusses profoundly the complications and the preventive methods associated.

In Chapter $\mathbf{3}$ and $\mathbf{4}$ the acute and long-term results of the 123-study are presented. In the 123-study the minimal cryoballoon application time necessary to achieve PVI and the possibility to reduce complication by shortening the application duration was assessed in a prospective and randomized fashion. These chapters assess the dosing aspect.

Chapter 5 assesses the incidence of low oesophageal temperatures in clinical practice during cryoballoon PVI. Possible predictive values for low oesophageal temperatures are verified in this chapter. This chapter assesses the monitoring as well as the predicting aspect.

Three-dimensional (3D) computed tomography (CT) overlay is a technique to create a live 3D image of the left atrium by integrating a previously obtained CT scan during fluoroscopy. Chapter 6 evaluates the benefits of 3D CT overlay in guiding cryoballoon PVI.

Chapter 7 describes the prevalence and consequences of incidental findings detected on CT prior to PVI and transcatheter aortic valve implantation.

In Chapter 8 the main findings of this thesis and their implications on clinical practice and on future research are discussed. 


\section{References}

1. Conen D. Epidemiology of atrial fibrillation. Eur Heart J. 2018;39(16):1323-1324.

doi:10.1093/eurheartj/ehy171

2. Go AS, Hylek EM, Phillips KA, et al. Prevalence of Diagnosed Atrial Fibrillation in Adults. JAMA. 2001;285(18):2370. doi:10.1001/jama.285.18.2370

3. Chiang CE, Naditch-Brûlé L, Murin J, et al. Distribution and risk profile of paroxysmal, persistent, and permanent atrial fibrillation in routine clinical practice insight from the real-life global survey evaluating patients with atrial fibrillation international registry. Circ Arrhythmia Electrophysiol. 2012;5(4):632-639. doi:10.1161/CIRCEP.112.970749

4. Oldgren J, Healey JS, Ezekowitz M, et al. Variations in cause and management of atrial fibrillation in a prospective registry of 15400 emergency department patients in 46 countries: The RE-LY atrial fibrillation registry. Circulation. 2014;129(15):1568-1576. doi:10.1161/CIRCULATIONAHA.113.005451

5. Nguyen TN, Hilmer SN, Cumming RG. Review of epidemiology and management of atrial fibrillation in developing countries. Int J Cardiol. 2013;167(6):2412-2420. doi:10.1016/j.ijcard.2013.01.184

6. Kannel W., Wolf P., Benjamin E., et al. Prevalence, incidence, prognosis, and predisposing conditions for atrial fibrillation: population-based estimates 11Reprints are not available. Am J Cardiol. 1998;82(7):2N-9N. doi:10.1016/S0002-9149(98)00583-9

7. Ball J, Carrington MJ, McMurray JJV, et al. Atrial fibrillation: Profile and burden of an evolving epidemic in the 21st century. Int J Cardiol. 2013;167(5):1807-1824. doi:10.1016/j.ijcard.2012.12.093

8. McManus DD, Rienstra M, Benjamin EJ. An update on the prognosis of patients with atrial fibrillation. Circulation. 2012;126(10):e143. doi:10.1161/CIRCULATIONAHA.112.129759

9. Zoni-Berisso M, Lercari F, Carazza T, et al. Epidemiology of atrial fbrillation: European perspective. Clin Epidemiol. 2014;6(1):213-220. doi:10.2147/CLEP.S47385

10. Fitzmaurice DA, Hobbs FDR, Jowett $S$, et al. Screening versus routine practice in detection of atrial fibrillation in patients aged 65 or over: Cluster randomised controlled trial. $\mathrm{Br}$ Med J. 2007;335(7616):383-386. doi:10.1136/bmj.39280.660567.55

11. Rho RW, Page RL. Asymptomatic atrial fibrillation. Prog Cardiovasc Dis. 2005;48(2):79-87. doi:10.1016/j.pcad.2005.06.005

12. Page RL, Wilkinson WE, Clair WK, et al. Asymptomatic arrhythmias in patients with symptomatic paroxysmal atrial fibrillation and paroxysmal supraventricular tachycardia. Circulation. 1994;89(1):224-227. doi:10.1161/01.cir.89.1.224

13. Stewart S, Hart CL, Hole DJ, et al. A population-based study of the long-term risks associated with atrial fibrillation: 20-year follow-up of the Renfrew/Paisley study. Am J Med. 2002;113(5):359364. doi:10.1016/S0002-9343(02)01236-6

14. Wijffels MCEF, Kirchhof CJHJ, Dorland R, et al. Atrial fibrillation begets atrial fibrillation: A study in awake chronically instrumented goats. Circulation. 1995;92(7):1954-1968. doi:10.1161/01.CIR.92.7.1954

15. Haïssaguerre M, Jaïs $P$, Shah DC, et al. Spontaneous initiation of atrial fibrillation by ectopic beats originating in the pulmonary veins. N Engl J Med. 1998;339(10):659-666. doi:10.1056/NEJM199809033391003

16. Weiss C, Gocht A, Willems S, et al. Impact of the distribution and structure of myocardium in the pulmonary veins for radiofrequency ablation of atrial fibrillation. PACE - Pacing Clin Electrophysiol. 2002;25(9):1352-1356. doi:10.1046/j.1460-9592.2002.01352.x 
17. Calkins $\mathrm{H}$, Reynolds MR, Spector $\mathrm{P}$, et al. Treatment of atrial fibrillation with antiarrhythmic drugs or radiofrequency ablation: two systematic literature reviews and meta-analyses. Circ Arrhythmia Electrophysiol. 2009;2(4):349-361. doi:10.1161/CIRCEP.108.824789

18. Bonanno C, Paccanaro M, La Vecchia L, et al. Efficacy and safety of catheter ablation versus antiarrhythmic drugs for atrial fibrillation: a meta-analysis of randomized trials. J Cardiovasc Med. 2010;11(6):408-418. doi:10.2459/JCM.0b013e328332e926

19. Laish-Farkash A, Suleiman M. Comparison of the Efficacy of PVAC ${ }^{\circledR}$ and nMARQTM for paroxysmal atrial fibrillation. J Atr Fibrillation. 2017;9(6). doi:10.4022/jafib.1550

20. Dukkipati SR, Cuoco F, Kutinsky I, et al. Pulmonary Vein Isolation Using the Visually Guided Laser Balloon A Prospective, Multicenter, and Randomized Comparison to Standard Radiofrequency Ablation. J Am Coll Cardiol. 2015;66:1350-1360. doi:10.1016/j.jacc.2015.07.036

21. Kuck K-HH, Brugada J, Fürnkranz A, et al. Cryoballoon or Radiofrequency Ablation for Paroxysmal Atrial Fibrillation. N Engl J Med. 2016;374(23):2235-2245. doi:10.1056/NEJMoa1602014

22. Roebuck JR. The Joule-Thomson Effect in Air. Proc Natl Acad Sci. 1926;12(1):55-58. doi:10.1073/pnas.12.1.55

23. Chun JKR, Bordignon S, Chen S, et al. Current Status of Atrial Fibrillation Ablation with Balloon Strategy. Korean Circ J. 2019;49(11):991. doi:10.4070/kcj.2019.0226

24. De Ponti R. Cryothermal energy ablation of cardiac arrhythmias 2005: State of the art. Indian Pacing Electrophysiol J. 2005;5(1):12-24.

25. Gage AA, Baust J. Mechanisms of Tissue Injury in Cryosurgery. Cryobiology. 1998;37(3):171-186. doi:10.1006/cryo.1998.2115

26. Cardoso R, Mendirichaga R, Fernandes G, et al. Cryoballoon versus Radiofrequency Catheter Ablation in Atrial Fibrillation: A Meta-Analysis. J Cardiovasc Electrophysiol. 2016;27(10):1151-1159. doi:10.1111/jce.13047

27. Ma H, Sun D, Luan $\mathrm{H}$, et al. Efficacy and safety of cryoballoon ablation versus radiofrequency catheter ablation in atrial fibrillation: an updated meta-analysis. Postep w Kardiol interwencyjnej = Adv Interv Cardiol. 2017;13(3):240-249. doi:10.5114/aic.2017.70196

28. Chen C, Gao X, Duan X, et al. Comparison of catheter ablation for paroxysmal atrial fibrillation between cryoballoon and radiofrequency: a meta-analysis. J Interv Card Electrophysiol. 2017;48(3):351-366. doi:10.1007/s10840-016-0220-8

29. Kirchhof P, Benussi S, Kotecha D, et al. 2016 ESC Guidelines for the management of atrial fibrillation developed in collaboration with EACTS. Eur Heart J. 2016;50(5):e1-e88. doi:10.1093/ejcts/ezw313

30. Liu X, Chen C, Gao X, et al. Safety and Efficacy of Different Catheter Ablations for Atrial Fibrillation: A Systematic Review and Meta-Analysis. Pacing Clin Electrophysiol. 2016;39(8):883899. doi:10.1111/pace.12889

31. Tomaiko E, Tseng A, Su WW. Radiofrequency versus cryoballoon ablation for atrial fibrillation. Curr Opin Cardiol. 2020;35(1):13-19. doi:10.1097/HCO.0000000000000700

32. Fürnkranz A, Bordignon S, Dugo D, et al. Improved 1-year clinical success rate of pulmonary vein isolation with the second-generation cryoballoon in patients with paroxysmal atrial fibrillation. J Cardiovasc Electrophysiol. 2014;25(8):840-844. doi:10.1111/jce.12417

33. Aytemir K, Gurses KM, Yalcin MU, et al. Safety and efficacy outcomes in patients undergoing pulmonary vein isolation with second-generation cryoballoon. Europace. 2014;17(3):379-387. doi:10.1093/europace/euu273

34. Guiot A, Savouré A, Godin B, et al. Collateral nervous damages after cryoballoon pulmonary vein 
isolation. J Cardiovasc Electrophysiol. 2012;23(4):346-351. doi:10.1111/j.1540-8167.2011.02219.x

35. Fürnkranz A, Bordignon S, Schmidt B, et al. Luminal esophageal temperature predicts esophageal lesions after second-generation cryoballoon pulmonary vein isolation. Heart Rhythm. 2013;10(6):789-793. doi:10.1016/j.hrthm.2013.02.021

36. Packer DL, Kowal RC, Wheelan KR, et al. Cryoballoon ablation of pulmonary veins for paroxysmal atrial fibrillation: first results of the North American Arctic Front (STOP AF) pivotal trial. J Am Coll Cardiol. 2013;61(16):1713-1723. doi:10.1016/j.jacc.2012.11.064

37. Ciconte G, de Asmundis C, Sieira J, et al. Single 3-minute freeze for second-generation cryoballoon ablation: One-year follow-up after pulmonary vein isolation. Heart Rhythm. 2015;12(4):673-680. doi:10.1016/j.hrthm.2014.12.026

38. Miyazaki $\mathrm{S}$, Hachiya $\mathrm{H}$, Nakamura $\mathrm{H}$, et al. Pulmonary vein isolation using a second-generation cryoballoon in patients with paroxysmal atrial fibrillation: One-year outcome using a single bigballoon 3-minute freeze technique. J Cardiovasc Electrophysiol. 2016;27(12):1375-1380. doi:10.1111/jce.13078

39. Heeger C-H, Wissner E, Wohlmuth P, et al. Bonus-freeze: benefit or risk? Two-year outcome and procedural comparison of a "bonus-freeze" and "no bonus-freeze" protocol using the secondgeneration cryoballoon for pulmonary vein isolation. Clin Res Cardiol. 2016;105(9):774-782. doi:10.1007/s00392-016-0987-8

40. De Regibus V, lacopino S, Abugattas JP, et al. Single freeze strategy with the second- generation cryballoon for atrial fibrillation: a multicenter international retrospective analysis in a large cohort of patients. J Interv Card Electrophysiol. 2017;49(2):173-180. doi:10.1007/s10840-017-0254-6

41. Tebbenjohanns J, Höfer C, Bergmann L, et al. Shortening of freezing cycles provides equal outcome to standard ablation procedure using second-generation $28 \mathrm{~mm}$ cryoballoon after 15month follow-up. Europace. 2016;18(2):206-210. doi:10.1093/europace/euv189

42. Chun KRJ, Stich M, Fürnkranz A, et al. Individualized cryoballoon energy pulmonary vein isolation guided by real-time pulmonary vein recordings, the randomized ICE-T trial. Heart Rhythm. 2017;14(4):495-500. doi:10.1016/j.hrthm.2016.12.014 
CHAPTER 2 


\section{SAFETY [FIRST] IN CRYOBALLOON AND RADIOFREQUENCY PULMONARY VEIN ISOLATION FOR ATRIAL FIBRILLATION}

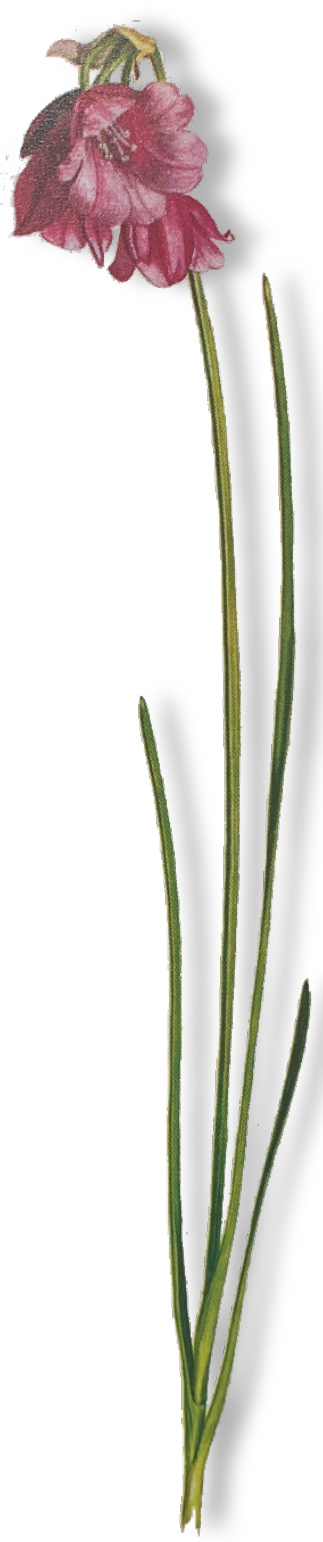




\section{Introduction}

Electrical isolation of the pulmonary veins (PVs) by ablation has become the cornerstone in symptomatic AF treatment?. Continuous improvement of ablation devices made catheter ablation the first-line therapy in selected patients, where radiofrequency (RF) and cryoballoon PVI are the recommended methods of use.

The main complications of PVI include thrombi and stroke, tamponade and damage to surrounding tissues ${ }^{2}$. Overall, the safety profiles of RF and cryoballoon PVI are comparable although there are some small differences. RF ablation is associated with a higher vascular complication rate and pericardial effusion or cardiac tamponade, whereas cryoballoon ablation is associated with higher rates of (phrenic) nerve injury ${ }^{3}$.

\section{PV Stenosis}

PV stenosis was the major complication of PVI in the early years of AF ablation ${ }^{4}$. PV stenosis can lead to dyspnea, chest pain, cough or haemoptysis 5 . Severe PV stenosis can progress to complete occlusion with potentially life-threatening symptoms of pulmonary hypertension or pulmonary venous infarction ${ }^{4,6}$. However, most cases of $\mathrm{PV}$ stenosis tend to be asymptomatic and symptoms can present aspecific as well.

PV stenosis has become a rare complication after changing the ablation strategy from ablating foci inside the PVs to a more antral approach, aided by intra-cardiac echocardiography and 3D mapping systems ${ }^{4,7}$. Nowadays the incidence of PV stenosis requiring intervention has been reported as $<1 \%$ after RF PVI. However, the diagnosis of PV stenosis by symptoms is challenging and the actual incidence rate can be underestimated ${ }^{8}$. Cryoballoon has even been suggested to have little to no risk of stenosis, with the FIRE AND ICE trial reporting an incidence of $0 \%$ in both RF and cryoballoon groups ${ }^{2,9}$.

\section{Thrombi and [silent] stroke}

The formation of thrombi and the associated risk of stroke has a reported incidence of $<1 \%$ in RF PVI. There are no significant differences between stroke in RF and cryoballoon $\mathrm{PVI}^{2,10}$. Despite the low incidence of stroke, consequences have a high impact on quality of life and morbidity. Therefore it deserves the utmost attention in clinical practice.

The low incidence of stroke is only present in adherence to a strict and periprocedural oral anticoagulation strategy. Even brief interruptions of OAC are 
associated with a significant increase in the risk of stroke or systemic embolism¹. The most recent European Society of Cardiology guidelines dictate that heparin should be given during the procedure in order to achieve an activated clotting time of $>300$ seconds. After PVI, procedure anticoagulation should be continued for at least 8 weeks in all patients ${ }^{12}$.

The periprocedural use of new oral anticoagulants (NOAC) for AF ablation is a controversial topic amongst electrophysiologists. The heterogeneity in periprocedural anticoagulation therapy in different practices among different countries is also part of the debate ${ }^{13}$. Several small observational studies and 3 randomized control trials have compared continuous periprocedural use of NOAC and VKA in PVI ${ }^{10,11,14}$. The meta-analyses performed on these studies suggest that continuation of NOACs is a safe, effective, and feasible approach during AF catheter ablation. In one of the two latest meta-analyses, a reduction in haemorrhagic complications while continuously using NOAC was found. Although these limited data are promising, the authors of these meta-analyses also conclude that the evidence available is of mediocre quality. They emphasize the need for more highquality randomized controlled trials to confirm their findings.

The major concern for electrophysiologists in performing PVI procedures with continued use of NOACs is a severe bleeding including cardiac tamponade. Results of a case series of 16 patients on uninterrupted NOACs and post-ablation tamponade highlights the critical importance of specific reversal agents for NOACS available during the AF ablation ${ }^{15,16}$.

The current standard in pre-procedural atrial thrombi detection involves two imaging tests; Transoesophageal echocardiography (TEE) and Computed Tomography (CT). Recent literature on this subject focuses on the necessity to use both modalities. The latest and largest studies suggest that TEE can be eliminated from routine practice and should only be used in patients with evidence for possible intra-cardiac thrombi on $\mathrm{CT}^{17-19}$.

Recent evidence suggests that clinical stroke is only a minority of all cerebral ischemic events. New ischemic lesions were identified on magnetic resonance imaging (MRI) in post-PVI patients without symptoms of clinical stroke. Due to the asymptomatic nature of these lesions they are referred to as "silent". The clinical relevance of silent cerebral infarctions (SCI) is unclear. Although it is suggested that these lesions might be viewed as surrogates for clinical stroke risk, no study has been able to link these lesions to neuropsychological decline ${ }^{20,21}$. It is certainly 
possible that a decline in cognitive function is multifactorial, related to the disease of AF itself, and not correlated to focal lesions created by AF ablation only.

Irrigated RF and cryoballoon PVI have similar SCI risks, with reported incidences up to $20 \%$ depending on the definition used ${ }^{20,22}$. Thus far no periprocedural monitoring system for $\mathrm{SCl}$ exists. A few studies have tried to detect periprocedural microembolic events by continuous transcranial Doppler signal registration. Use of the conventional RF catheter for PVI was associated with a significantly higher incidence of cerebral microembolic signals compared to the irrigated RF tip catheter or the cryoballoon catheter ${ }^{23}$. However, a relationship between the occurrence of periprocedural microembolic events with transcranial doppler and the detection of $\mathrm{SCl}$ in MRI after the procedure is not reported yet ${ }^{24,25}$. The majority of acute MRI lesions observed after AF ablation showed spontaneous regression without evidence of chronic glial scar at short term follow-up ${ }^{20}$.

\section{Tamponade}

Tamponade is a serious adverse event which can occur during transseptal puncture or during the ablation itself when too much energy, pressure, cold or heat, is delivered at a single spot. The risk of tamponade is significantly higher in RF compared to cryoballoon PVI (1.3 vs $0.5 \%$ ), which can be explained by several factors $^{26,27}$. Lesions created using RF involve some degree of endothelial disruption which increases the risk for perforation and thrombogenicity. Although this risk is small for focal lesions, the risk increases for procedures requiring extensive lesions. If temperatures $>100{ }^{\circ} \mathrm{C}$ are reached even coagulum formation on the tip of the RF catheter can occur, which can ultimately lead to steam pop formation. In contrast, the destruction of tissue by cryo results in minimal tissue disruption and preserves basic underlying tissue architecture. As a result these lesions have good tensile strength and are less thrombogeni ${ }^{28,29}$.

With the introduction of contact force (CF) sensing catheters in RF PVI, the catheter-tissue CF can be measured hence minimising the likelihood of applying too little as well as too much force ${ }^{30}$. Despite the fact that safety and applicability of CF catheters are demonstrated, the translation of real-time CF monitoring during RF ablation into clinical benefit is still controversial ${ }^{31-33}$. Although prospective randomised clinical studies with longer follow up are lacking, RF ablation guided by real-time CF sensing is now the dominant ablation modality, particularly for catheter ablation of AF. A recent meta-analysis shows not only an improved efficacy while using CF catheters but also a significantly lower incidence of complications ${ }^{34-}$ 
36. Randomized controlled studies are required to assess whether catheter ablation using an optimized CF improves the long-term clinical outcome.

The creation of sufficient RF lesions is limited by the possible adverse effects of rising impedances at the catheter tip. Rising impedances are related to the development of soft thrombus and steam pop and can be prevented by active cooling of the electrode-tissue interface ${ }^{37}$. With active cooling, higher quantities of RF power can be delivered for a longer period of time, which results in larger lesions with greater depth ${ }^{37}$. The improvement of the efficacy of RF PVI procedures by developing irrigated ablation catheters, therefore, was secondary to the improvement of the safety.

In 2009 the first open irrigated catheter was approved for ablation. It had 6 irrigation holes and was recently improved with 56 irrigation holes (Thermocool Surround Flow, Biosense Webster, Diamond Bar, CA, USA) (SF catheter). This catheter provides more uniform cooling over the entire tip rather than localized cooling at the distal tip. Delivery of high RF power, even in areas of very low blood flow, is now possible and irrigation flow rate is reduced ${ }^{38}$. However, the initial enthusiasm about this catheter diminished after a recently published observational prospective study. This study shows an increase in unpredictable incidence of steam pop formation using the SF catheter despite different power and irrigation settings $\mathrm{s}^{39}$. Due to the superficial, endocardial, tissue cooling the highest tissue temperature using the SF catheter is achieved in the sub-endocardial layers. This can result in temperature disparities between the catheter tip and the tissue during RF delivery. It has been shown that the electrode temperature and impedance, both used to monitor tissue feedback in response to ablation in conventional ablation, are not predictive for the occurrence of steam pops in SF catheters.

Simultaneous CF measurement has been suggested to overcome this limitation of SF catheters by adjusting the energy according to the CF applied to the tissue. A novel RF ablation catheter incorporating both technologies, CF sensing as well as porous tip irrigation (Thermocool SmartTouch SurroundFlow, Biosense Webster, Diamond Bar, CA, USA), has been released recently. It showed promising results in safety and efficacy and requires further clinical evaluation ${ }^{40-42}$.

To prevent tamponade during transseptal puncture the use of echocardiographic guidance allows the direct visualization of the needle in the fossa ovalis. Besides a safe puncture it is possible to visualise the optimal puncture site for ablation, 
which can make a significant difference in catheter manoeuvrability and mapping options $^{43}$.

A possible side effect of conventional TEE is the occurrence of an oesophageal hematoma (incidence 0.27\%). However with careful introduction and immediate removal of the probe after transseptal puncture this complication can be prevented. Alternatives to the conventional TEE for imaging during transseptal puncture are now available in the Intra Cardiac Echocardiography (ICE) method and the more recently developed micro and mini-TEE probes. These alternatives aim to overcome oesophageal hematoma as a complication ${ }^{44}$.

With the traditional rigid angulated Brockenbrough needle, mechanical pressure is needed to perform the transseptal puncture. This is a challenge, especially in the presence of a thickened fibrotic or aneurysmatic septum together with the related risk of overshooting in normal-sized or moderately dilated left atria. To overcome this challenge, flexible RF powered needles are developed. They have shown improvement in the safety and efficacy of transseptal punctures in daily practice $^{45,46}$.

To facilitate safe and accurate navigation of the instruments during PVI itself, several techniques of image integration are available. With the use of 3D overlay, a live 3D image is created during the procedure by integrating fluoroscopy with a novel rotational angiographic 3D image or a previously made CT or MRI image of the left atrium. These techniques demonstrate their advantage in more straightforward manoeuvring and placing of the balloon or catheters ${ }^{47}$. For cryoballoon PVI it can facilitate optimal positioning of the balloon, and therefore a significant reduction in the contrast and radiation dose and possibly the necessity for extra applications ${ }^{48}$. Furthermore, CT or MRI imaging pre-PVI can contribute to a better understanding of the anatomy of the PVs, so the operators can select the appropriate ablation technique and achieve maximum efficacy of $\mathrm{PV}{ }^{49}$.

Also non-fluoroscopic 3D navigation methods are developed, where the position of catheters is determined by magnetic or impedance tracking and displayed on a perprocedural acquired 3D swap of the left atrium or a pre-procedural CT or MRI image (Carto system (Biosense-Webster), EnSite NavX system (Abbott), Rhythmia system (Boston Scientific). These methods show a high level of accuracy in location of the catheters and avoid the use of fluoroscopy during the procedure ${ }^{50-52}$. One of the main advantages of the 3D system is to mark catheter positions on the endocardial 
surface to which the operator can return after exploring other points which is not possible using fluoroscopy ${ }^{53}$.

Merging cardiac CT or MRI data with electroanatomic mapping of the left atrium is now common practice. However, none of these modalities offers real-time imaging at the time of the procedure and, due to the time between the scan and the actual ablation procedure, there can be potential changes in the shape and position of the left atrium. Recently several protocols for accurate integration of real-time 2D ICE and a CT reconstruction using a 3D mapping system are tested and validated. The results are promising, but further studies must show if it can reduce radiation exposure and improve procedural safety ${ }^{54}$.

\section{Damage to surrounding tissues}

When cryo- or RF energy is delivered to the atrial wall and the PVs, the surrounding tissue is affected as well. The energy spreads beyond the heart to critical structures as the lungs, oesophagus, phrenic and vagal nerves. The distribution of energy in surrounding tissues can be very extensive. Complications attributed to collateral damage are oesophageal ulcera and fistula, gastroparesis, phrenic nerve palsy and pulmonary complications.

\section{Bronchial effects}

Bronchial damage is reported as a possible, albeit infrequent, complication of cryoballoon PVI. Bronchial complications for cryoballoon ablation include persistent cough (incidence reported up to 17\%), haemoptysis and even fatal atrio-bronchial fistula ${ }^{55,56}$. Bronchial complications in RF PVI and PV stenosis have significantly been reduced by the earlier mentioned more antral ablation technique and the use of 3D mapping systems.

There is some debate about the mechanism of pulmonary complications after cryoballoon PVI. Thermal injury, like the formation of ice in the bronchus, shown in bronchoscopy recordings during or after cryoballoon PVI, is repeatedly reported in literature ${ }^{56,57}$. The possible formation of ice outlines the power of the technique, and therefore the risk for complications in surrounding tissues. A recent prospective study showed a high incidence of unintentional cryoablation of the left main bronchus with even endobronchial bleeding in bronchial endoscopy recordings. Despite this high incidence, none of the patients developed haemoptysis or cough, so the long-term clinical consequences still need to be investigated ${ }^{56}$. 
A number of safety measures to prevent bronchial effects of cryoballoon PVI are suggested in literature and (partially) adapted in common clinical practice. Prevention is realized by means of minimizing excessive force during occlusion of the PV. For instance by the use of different techniques such as the pull-down manoeuvre, to avoid applications deep in the PV, and temperature monitoring ${ }^{56,58}$. since there is no relation between low balloon temperatures and bronchial damage, intraluminal endobronchial temperature monitoring has been suggested instead of balloon temperature monitoring ${ }^{56}$.

Another easily applicable safety measurement to prevent collateral damage is changing the dosing protocol, for instance in cryoballoon PVI by shortening the application duration. Historically a two times four minutes approach is adopted. Recent studies show that shorter application durations do not compromise the efficacy of the procedure while the exposure to surrounding tissue is limited. However, the optimal freezing duration has not been determined yet ${ }^{59,60}$.

\section{Oesophageal related complications}

The most devastating complication affecting the surrounding tissue of the heart is an atrio-oesophageal fistula (AEF). The estimated incidence is 1 in 500-1000 for RF $\mathrm{PVI}$ and 1 in 10000 for cryoballoon PVI, but it is associated with $>50 \%$ mortality ${ }^{61}$. Although the exact mechanism is unclear, direct cooling or heating of surrounding tissue plays a major role in the formation of AEF. Other mechanisms include injury of the oesophageal vasculature with late oesophageal necrosis or ischemic oesophageal injury ${ }^{62}$.

The occurrence of AEF is a late complication. The most common time of presentation is 2 to 4 weeks post-procedure, but it can occur up to 2 months postablation. For this reason it can be underreported in studies. Most of the patients present with delayed post-ablation fever or sepsis with or without neurological or gastrointestinal symptoms. The various clinical presentations of this complication may lead to a delay in diagnosis. Mortality in these patients is extremely high when treated conservatively and can be significantly decreased with surgical repair. Even with surgical therapy one-third of the fistula's are lethal ${ }^{61,63}$.

The vagal nerve is situated closely to the heart and spreads out on the oesophagus in a web-like structure. It innervates the stomach, to provide gastric motility, and controls the pyloric sphincter. Injury to the vagal nerve due to PVI may cause gastroparesis, a complication characterized by delayed gastric emptying in absence of mechanical obstruction of the stomach. Symptoms include nausea, vomiting, 
bloating, abdominal pain and gastric discomfort ${ }^{64}$. Although not mentioned very often, gastroparesis is a relatively common complication after ablation because of collateral nerve damage ${ }^{65}$. The incidence is probably higher than direct oesophageal injury ${ }^{66}$. A recent meta-analysis shows no significant difference in the incidence of symptoms associated with gastroparesis after RF or cryoballoon PVIs (3.2 vs $2.1 \%)^{67}$. However, not all patients with proven gastroparesis developed symptoms, possibly because of a quick recovery ${ }^{68}$. Patients do not always relate their gastric problems to the cardiac procedure which is one of the reasons for possible underreporting of this complication.

With the first-generation cryoballoon, thermal oesophageal lesions as well as associated AEFs and gastroparesis due to vagal nerve injury, were rarely reported. However, after the introduction of the second-generation cryoballoon, the incidence of thermal oesophageal lesions and the number of gastropareses increased ${ }^{69}$. Studies in which systematic postprocedural oesophagoscopy was performed, reported an increased incidence of oesophageal ulcerations with the use of the second-generation cryoballoon (13.4\%) compared to the first-generation cryoballoon ${ }^{70,71}$. Recent studies also showed an incidence of up to $48 \%$ of asymptomatic endoscopically detected oesophageal lesions after RF PVI'

A fivefold higher chance for AEFs was reported with the use of CF catheters, compared to non-CF catheters ${ }^{73}$. With CF catheters, operators are also enabled to ablate for a longer period of time and using greater force. Studies showed a forcetime integral, a measure introduced to reflect the force applied over a certain time, of $400 \mathrm{gs}$ per lesion as a target value to prevent reconduction. Considering the variation in wall thickness in the left atrium, this value may be too high for the thinner posterior atrial wall ${ }^{33}$. The absence of strong data to guide ablation on the posterior wall is a significant obstacle to optimize efficacy and safety of ablation for atrial fibrillation.

Oesophageal temperature (OT) monitoring has been introduced as a safety measure to prevent collateral damage to the oesophagus and the vagal nerve. Several thermoprobes have been developed to monitor the OT. Starting from single, non-insulated, non-steerable sensors to multi-sensor, insulated probes that cover a large area of the oesophageal wall. The first thermoprobes were manually repositioned into a position close to the point of ablation. These probes were heavily debated because of possible excess OT due to ohmic heating during RF PVI and the possible underestimation of the ET. This possible under-estimation of the OT was believed to be caused by the position of the probe. It could be positioned 


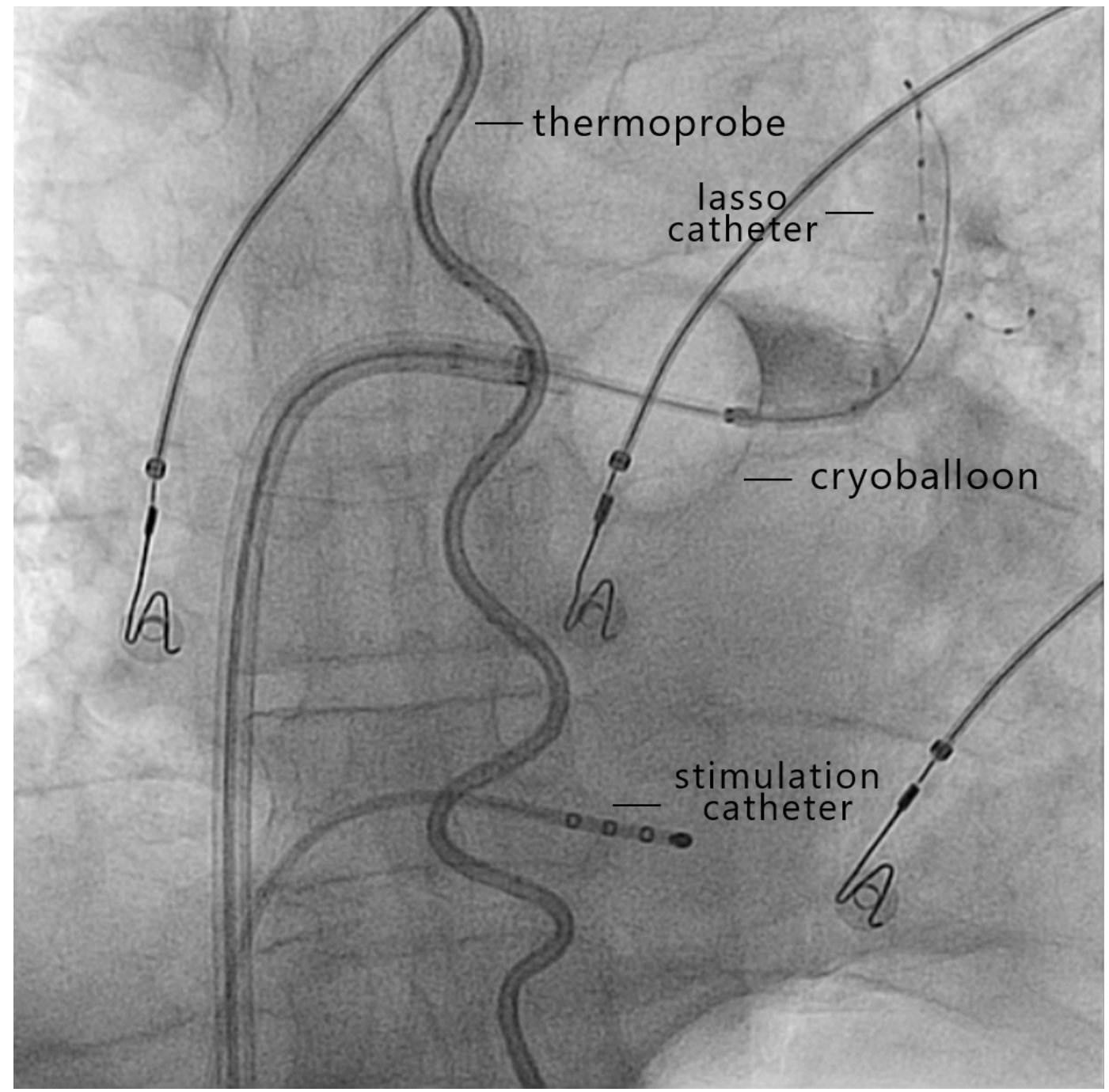

Figure 1 Position of the thermoprobe (s-shaped) in the oesophagus with cryoballoon positioned at the antrum of the left superior pulmonary vein with the lasso catheter inside this pulmonary vein and contrast dye being injected in the pulmonary vein (right upper quadrant). The stimulation catheter is positioned in the coronary sinus. 
contralateral to the side where the energy is applied or could be floating free in the oesophagus ${ }^{74,75}$. With the introduction of the most recent thermoprobe (Circa SCath $^{\text {TM }}$, CIRCA Scientific, CO, USA), some of these disadvantages seem to be eliminated ${ }^{76}$. Insulation prevents the probe from possible ohmic heating and it contains 12 electrically insulated temperature sensors. Due to the S-shape of the probe it covers the whole length of the oesophagus in relation to the PVs which prevents the need to reposition the probe during the procedure (Figure 1).

This OT guiding significantly reduces the incidence of thermal oesophageal lesions in cryoballoon PVI. An OT $\leq 12^{\circ} \mathrm{C}$ in cryoballoon PVI can predict the formation of gastro-oesophageal lesions with a sensitivity of $100 \%$ and specificity of $92 \%{ }^{71}$. Since the OT decreases even after interruption of the cryoapplication, an interruption temperature higher than $12^{\circ} \mathrm{C}$ is advised ${ }^{7}$. Interruption of cryoapplication at OTs of at least $15^{\circ} \mathrm{C}$ or even higher is advised $d^{78}$. These cut-off values have been established with the use of a thermoprobe with 3 thermocouples, although it is assumed that these values can be extrapolated to the most recent Circa probe. In RF PVI procedures where OT is measured, a reduction of the incidence of vagal nerve complications and oesophageal injury has been reported ${ }^{79,80}$. Using RF a cut-off value of $39^{\circ} \mathrm{C}$ is suggested to prevent collateral damage, however the optimal cutoff value still needs confirmation ${ }^{79}$.

In our centre, routine OT monitoring is performed in every PVI procedure, both for $\mathrm{RF}$ and cryoballoon PVI. At first a thermoprobe was used with 3 thermocouples separated by $10 \mathrm{~mm}$ (SensiTherm ${ }^{\mathrm{T}}$, St Jude Medical Inc. MN, USA). It was adjusted the position of the temperature probe to the fluoroscopic position of the balloon. Since February 2015 we have been using the Circa probe. When OT falls below $16^{\circ} \mathrm{C}$ in cryoballoon and reaches $>39^{\circ} \mathrm{C}$ in RF, the application is stopped prematurely. A subsequent application is not performed until OT reaches $>30^{\circ} \mathrm{C}$ in cryoballoon and $<38^{\circ} \mathrm{C}$ in RF to avoid a stacking effect.

In our registration of 204 patients in whom cryoballoon therapy has been performed with a thermoprobe in situ, we found a high incidence of low OT, temperatures below $20^{\circ} \mathrm{C}$ in $26 \%$ and below $16^{\circ} \mathrm{C}$ in $13 \%$ of the patients (Figure 2). Low OTs were only found in the left and right inferior PVs. No clinically significant correlation between minimum balloon temperature and lowest OT was found which is in accordance with earlier reports (Figure 3$)^{71,78,81}$. Therefore the use of balloon temperature alone as a surrogate for OT is not reliable. 


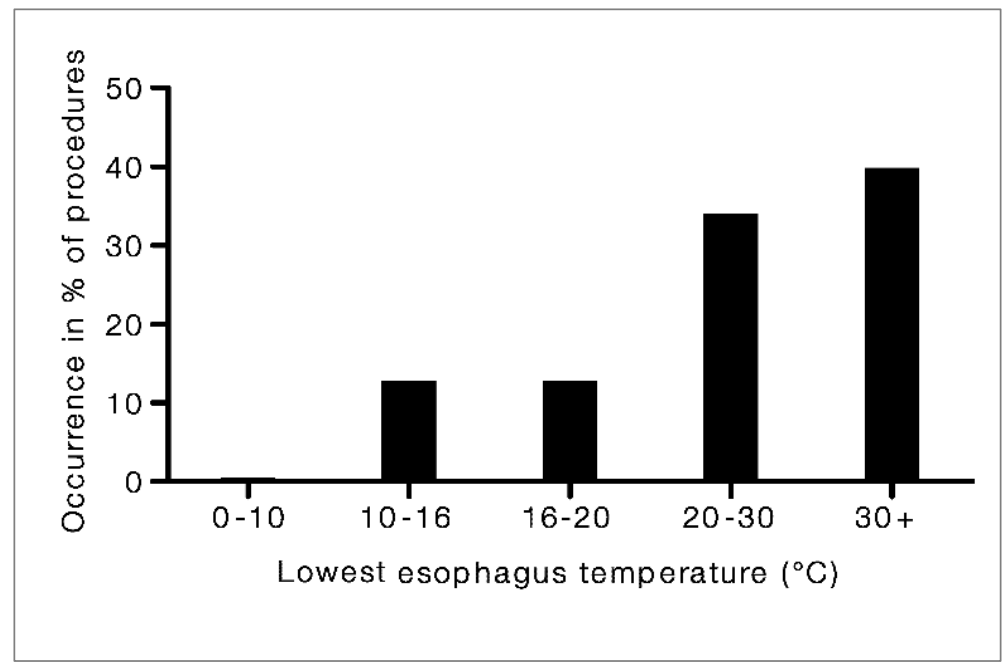

Figure 2 Occurrence (in \% of procedures) of lowest oesophagus temperature $\left({ }^{\circ} \mathrm{C}\right.$ )

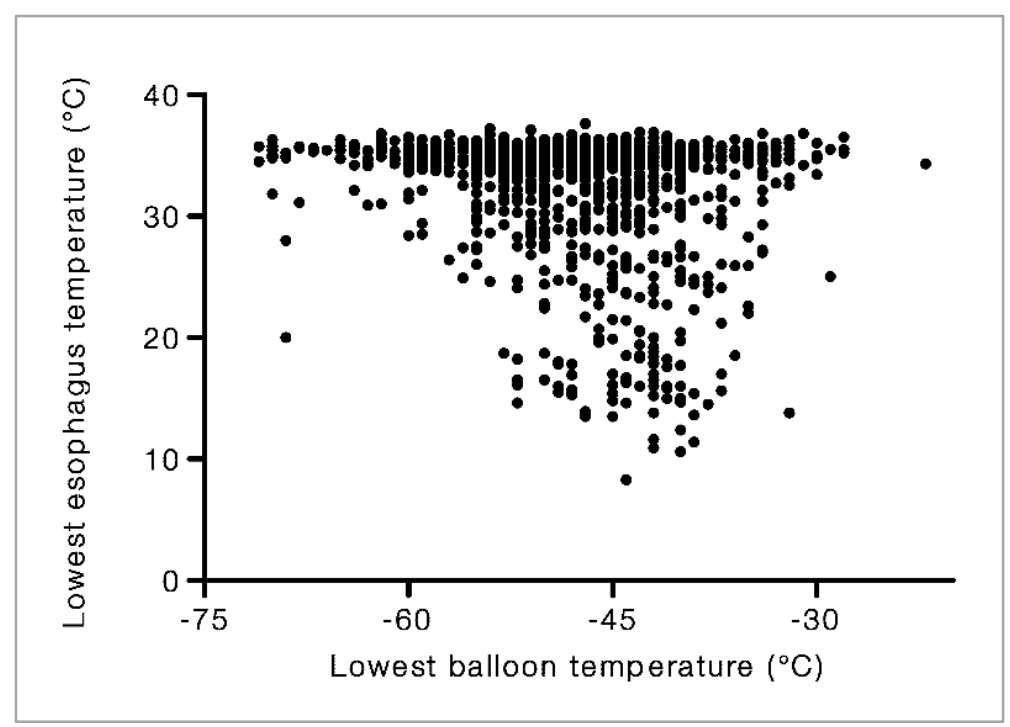

Figure 3 Relation between lowest oesophagus temperature and lowest balloon temperature 
Other suggestions of preventative measures for AEF are oesophageal cooling, posterior displacement of the oesophagus with the TEE probe, and the use of prophylactic proton pump inhibitor ${ }^{82-84}$. However, the effectiveness of these oesophageal protection strategies has not been proven and the occurrence of AEF in cases despite preventive strategies demonstrate that AEF prevention is not guaranteed $^{61}$. Therefore clinical alertness in the post-operative period will remain an important strategy in the early diagnose of AEFs. A recent study documented that an initial thermal oesophageal lesion is a starting point of a cascade leading to AEF. This demonstrated that 1 out of 10 post-ablation oesophageal ulcers will progress to perforations, therefore post-ablation endoscopy can identify patients at high risk for oesophageal perforation ${ }^{85}$. Further and technical improvements are necessary to define preventive measures to minimize oesophageal injury.

\section{Phrenic nerve palsy}

Another critical structure positioned close to the heart is the phrenic nerve. Originating from the third to fifth cervical nerves it is situated outside the pericardium anterior of the pulmonary veins and provides the only motor output to the diaphragm. Diaphragmal excursion during spontaneous breathing depends on this nerve. Its close proximity to the right superior PV renders it vulnerable during ablation of this $\mathrm{PV}^{86}$.

The incidence of reported phrenic nerve palsy varies widely but is undoubtedly more common in cryoballoon PVI than in RF PVI. A recent meta-analysis showed a $6.6 \%$ vs $0.1 \%$ incidence of phrenic nerve palsy for cryoballoon vs RF PVI. Almost all cases of phrenic nerve palsy resolved during follow-up ${ }^{27}$. Persistent phrenic nerve palsy has recently been reported in a meta-analysis which shows a $1.7 \%$ vs $0.01 \%$ incidence for cryoballoon vs RF PVI'

Several strategies to avoid phrenic nerve palsy have been described. Continuous phrenic nerve stimulation combined with manual diaphragmal contraction surveillance is the most commonly used method in clinical practice. Since the close proximity of the phrenic nerve to the superior vena cava at the anterolateral junction, the phrenic nerve can be stimulated by capturing it from the superior vena cava for cryoballoon $\mathrm{PVI}{ }^{87}$. As an alternative the phrenic nerve can also be paced from the right subclavian vein, resulting in lower capture thresholds and possibly less catheter instability in clinical practice. As an anatomical variation, the left phrenic nerve can run down the left superior PV, this is discovered by pacing all the electrode pairs of the lasso catheter in this PV. If this anatomical variation is found, the phrenic nerve can be paced from the left subclavian vein ${ }^{88,89}$. For RF PVI, 
the phrenic nerve can be stimulated from the lasso or the ablation catheter itself when ablation is performed at a region in which the proximity of the phrenic nerve is expected.

If the operator observes a decrease or disappearance of diaphragmatic contractions the application is ceased immediately, irrespective of which PVI method used. In cryoballoon PVI direct deflation of the balloon is achieved using the double-stop technique, resulting in immediate cessation of refrigerant flow and deflation of the balloon where merely a single stop stops the refrigerant flow ${ }^{90}$. With the use of this manual diaphragm motion surveillance technique, the phrenic nerve is usually affected for a short period and function returns to normal within minutes to hours. However, while using this technique still a substantial number of patients cannot be protected from long-lasting phrenic nerve palsy.

A small number of studies have reported the use of another rather simple additional technique to monitor phrenic nerve function. In this technique the function of the phrenic nerve is measured by compound motor action potentials (CMAPS) obtained from two regular surface ECG electrodes. The basic principle of measuring CMAPs to prevent phrenic nerve palsy was first described in dogs and was followed by human evaluation ${ }^{91,92}$. Since a decrease in CMAP precedes the reduction in diaphragmic motion, it enables the operator to stop the application in an early phase. By using this technique a marked reduction in acute and persistent hemi-diaphragmatic paralysis and less severe histological damage to the phrenic nerve using CMAP has been shown ${ }^{93}$.

Figure 5 demonstrates the technique: two surface electrodes are positioned $5 \mathrm{~cm}$ above the xiphoid process and $16 \mathrm{~cm}$ along the right costal margin for monitoring the right diaphragmatic CMAPs. CMAP amplitudes are measured from peak to peak which is combined with manual diaphragm motion surveillance during the procedure. Clinical studies with 50-200 patients show a significant decrease in phrenic nerve palsy when manual surveillance only is compared to manual surveillance combined with CMAP94. In all patients with a reduction in diaphragmatic motion a significant decrease in CMAP was seen ${ }^{95,96}$.

The exact methodology of performing CMAP differs slightly between the aforementioned clinical studies. A variety of pacing outputs ranging from 5-20mA and $1-2.9 \mathrm{~ms}$ are used and show that the use of lower pacing outputs results in earlier detection of phrenic nerve palsy ${ }^{97}$. Furthermore, the cut-off value of CMAP decline which is used as a predictor for phrenic nerve palsy varies slightly from $30-$ 


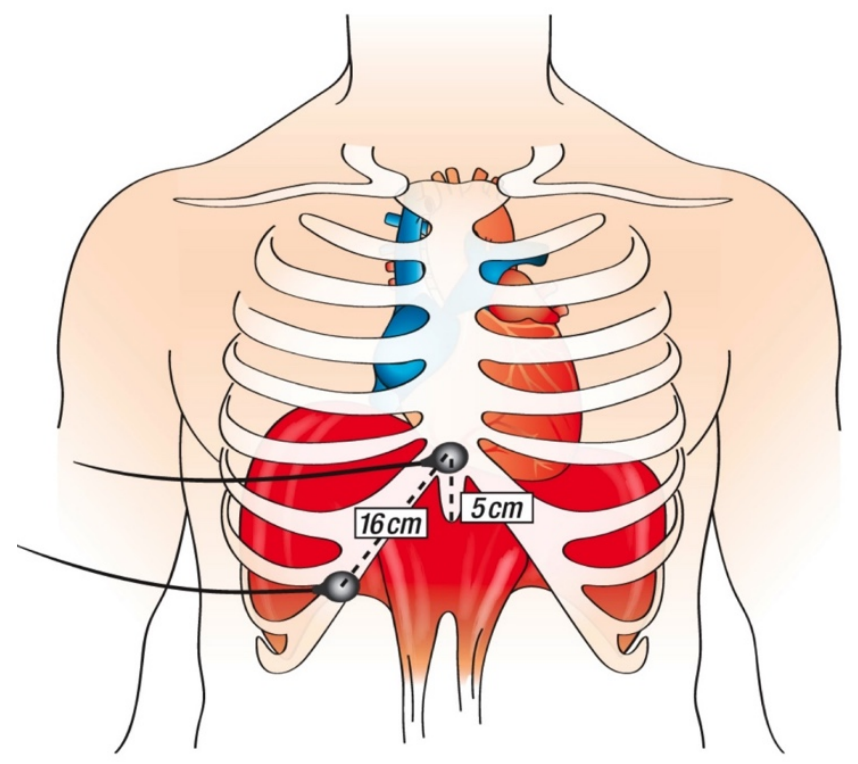

Figure 4 Position of surface electrodes (Reprinted from Franceschi et al. ${ }^{94}$ with permission of the publisher)

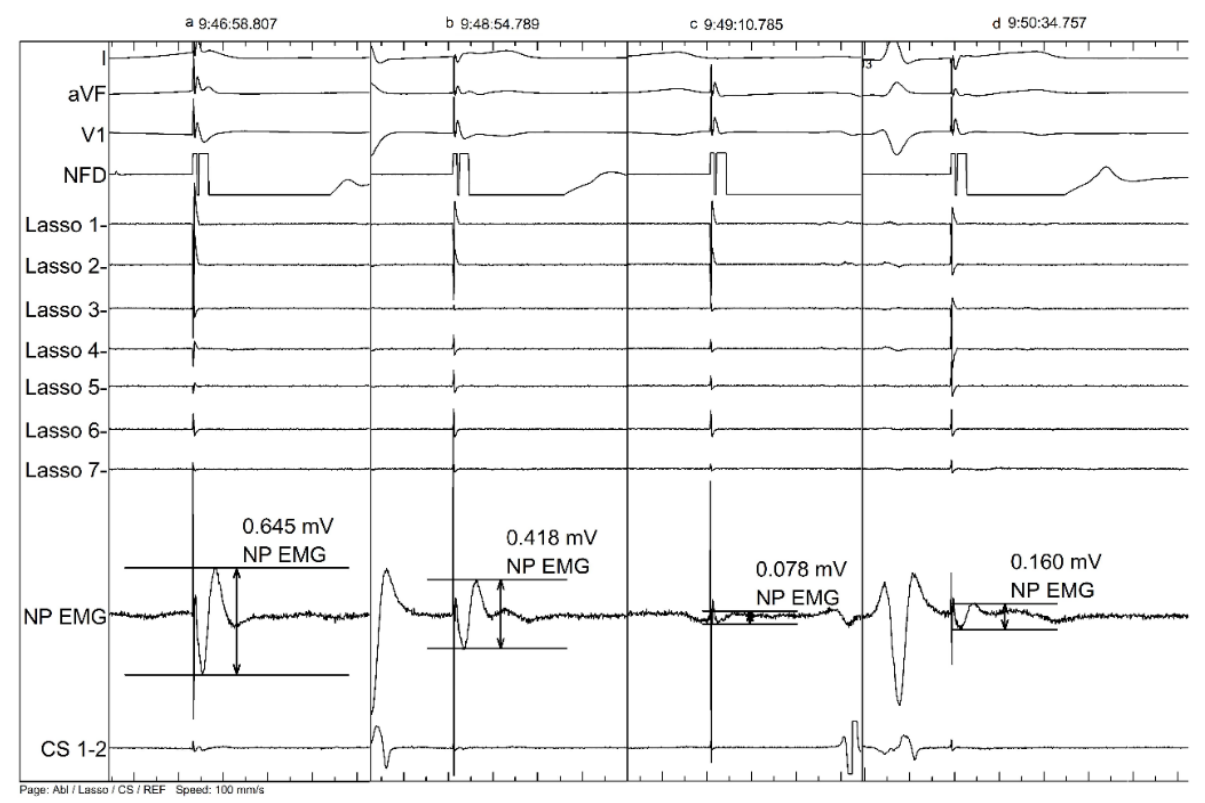

Figure 5 CMAP measurements of the second patient with a) Baseline CMAP b) Application stopped at 35\% decrease of (MAP c) Complete absence of a CMAP amplitude 15 seconds after discontinuation, along with the absence of diaphragmatic contraction d) Incomplete recovery of CMAP 
$35 \%$. The method and extent of anaesthesia differed between the different studies as well as the used procedure for acute abortion of freezing (double stop vs single stop). Measuring the CMAPs can be an additional safety measure and is described as a reliable, cheap, easy and sensitive method for predicting phrenic nerve palsy without compromising the procedural effectiveness ${ }^{96}$.

Figure 5 shows an example of the usefulness of CMAP during cryoballoon isolation of the right superior PV in our centre. PVI was performed under general anaesthesia using the second-generation (Arctic Front Advance ${ }^{\mathrm{TM}}$ Medtronic Inc., MN, USA) cryoballoon. No paralytic agents that inhibiting phrenic nerve capture had been administered. During cryoablation of the right PVs, the phrenic nerve was continuously stimulated by pacing on the quadripolar catheter positioned in the superior vena cava. The pacing site was carefully selected, guided by a stable pacing capture. The pacing threshold was tested and pacing was performed at twice the threshold value for a duration of $2 \mathrm{~ms}$ using a cycle length of $1500 \mathrm{~ms}$.

During cryoballoon application in the right superior PV, a decrease in CMAP of 35\% was noticed 114 seconds after the start of the ablation. At that moment the application was stopped using the double stop technique, no change in diaphragm excursion was noticed. However, the CMAP amplitude continued to decrease after discontinuation of the ablation resulting in complete absence of a CMAP amplitude 15 seconds after discontinuation, along with the absence of diaphragmatic contraction. The CMAP did recover at the end of the procedure, albeit not completely.

\section{Conclusion}

Both RF and cryoballoon PVI are effective methods to achieve PVI. All efforts should be made to increase the safety of the procedure for the patient. A broad range of safety measures is available. Both CMAP and measurement of OT can enhance the safety profile of the procedure. Limiting the amount of RF- and cryoenergy while maintaining efficacy is an ongoing subject of investigation ${ }^{60}$. 


\section{References}

1. Haïssaguerre $M$, Jaïs $P$, Shah DC, et al. Spontaneous Initiation of Atrial Fibrillation by Ectopic Beats Originating in the Pulmonary Veins. N Engl J Med. 1998;339(10):659-666. doi:10.1056/NEJM199809033391003

2. Kuck K-HH, Brugada J, Fürnkranz A, et al. Cryoballoon or Radiofrequency Ablation for Paroxysmal Atrial Fibrillation. N Engl J Med. 2016;374(23):2235-2245. doi:10.1056/NEJMoa1602014

3. Garg J, Chaudhary R, Palaniswamy C, et al. Cryoballoon versus Radiofrequency Ablation for Atrial Fibrillation: A Meta-analysis of 16 Clinical Trials. J Atr Fibrillation. 2016;9(3):1429. doi:10.4022/jafib.1429

4. Edriss H, Denega T, Test V, et al. Pulmonary vein stenosis complicating radiofrequency catheter ablation for atrial fibrillation: A literature review. Respir Med. 2016;117:215-222. doi:10.1016/j.rmed.2016.06.014

5. Pürerfellner $\mathrm{H}$, Aichinger J, Martinek $\mathrm{M}$, et al. Incidence, management, and outcome in significant pulmonary vein stenosis complicating ablation for atrial fibrillation. Am J Cardiol. 2004;93(11):1428-1431. doi:10.1016/j.amjcard.2004.02.049

6. Ravenel JG, McAdams HP. Pulmonary Venous Infarction After Radiofrequency Ablation for Atrial Fibrillation. Am J Roentgenol. 2002;178(3):664-666. doi:10.2214/ajr.178.3.1780664

7. Rostamian A, Narayan SM, Thomson L, et al. The incidence, diagnosis, and management of pulmonary vein stenosis as a complication of atrial fibrillation ablation. J Interv Card Electrophysiol. 2014;40(1):63-74. doi:10.1007/s10840-014-9885-z

8. Cappato R, Calkins H, Chen S-A, et al. Updated worldwide survey on the methods, efficacy, and safety of catheter ablation for human atrial fibrillation. Circ Arrhythm Electrophysiol. 2010;3(1):3238. doi:10.1161/CIRCEP.109.859116

9. Mugnai G, de Asmundis C, Ciconte G, et al. Incidence and characteristics of complications in the setting of second-generation cryoballoon ablation: A large single-center study of 500 consecutive patients. Heart Rhythm. 2015;12(7):1476-1482. doi:10.1016/j.hrthm.2015.04.001

10. Cardoso R, Knijnik L, Bhonsale A, et al. An updated meta-analysis of novel oral anticoagulants versus vitamin $\mathrm{K}$ antagonists for uninterrupted anticoagulation in atrial fibrillation catheter ablation. Heart Rhythm. 2018;15(1):107-115. doi:10.1016/j.hrthm.2017.09.011

11. Zhao $Y$, Yang $Y$, Tang $X$, et al. New oral anticoagulants compared to warfarin for perioperative anticoagulation in patients undergoing atrial fibrillation catheter ablation: a meta-analysis of continuous or interrupted new oral anticoagulants during ablation compared to interrupted or co. J Interv Card Electrophysiol. 2017;48(3):267-282. doi:10.1007/s10840-016-0221-7

12. Kirchhof P, Benussi S, Kotecha D, et al. 2016 ESC Guidelines for the management of atrial fibrillation developed in collaboration with EACTS. Eur Heart J. 2016;50(5):e1-e88. doi:10.1093/ejcts/ezw313

13. Potpara TS, Larsen TB, Deharo JC, et al. Oral anticoagulant therapy for stroke prevention in patients with atrial fibrillation undergoing ablation: results from the First European Snapshot Survey on Procedural Routines for Atrial Fibrillation Ablation (ESS-PRAFA). EP Europace. 2015;17:986-993. doi:10.1093/europace/euv132

14. Elgendy AY, Mahtta D, Barakat AF, et al. Meta-Analysis of Safety and Efficacy of Uninterrupted Non-Vitamin K Antagonist Oral Anticoagulants Versus Vitamin K Antagonists for Catheter Ablation of Atrial Fibrillation. J Cardiol. 2017;120:1830-1836. doi:10.1016/j.amjcard.2017.07.096

15. Gianni C, Di Biase L, Mohanty S, et al. Management of periprocedural and early pericardial effusions with tamponade following ablation of atrial fibrillation with uninterrupted factor Xa 
inhibitors: A case series. J Cardiovasc Electrophysiol. 2016;27(4):399-403. doi:10.1111/jce.12918

16. Mujer MTP, Rai MP, Atti V, et al. An Update on the Reversal of Non-Vitamin K Antagonist Oral Anticoagulants. Adv Hematol. 2020;2020:1-10. doi:10.1155/2020/7636104

17. Munir S, Chang JH, Salahudeen SR, et al. Atrial thrombi detection prior to pulmonary vein isolation: Diagnostic accuracy of cardiac computed tomography versus transesophageal echocardiography. Cardiol J. 2015;22(5):576-582. doi:10.5603/CJ.a2015.0017

18. Martinez MW, Kirsch J, Williamson EE, et al. Utility of Nongated Multidetector Computed Tomography for Detection of Left Atrial Thrombus in Patients Undergoing Catheter Ablation of Atrial Fibrillation. JACC Cardiovasc Imaging. 2009;2(1):69-76. doi:10.1016/j.jcmg.2008.09.011

19. Romero J, Husain SA, Kelesidis I, et al. Detection of Left Atrial Appendage Thrombus by Cardiac Computed Tomography in Patients With Atrial Fibrillation. Circ Cardiovasc Imaging. 2013;6(2):185194. doi:10.1161/CIRCIMAGING.112.000153

20. Deneke T, Jais P, Scaglione M, et al. Silent cerebral events/lesions related to atrial fibrillation ablation: a clinical review. J Cardiovasc Electrophysiol. 2015;26(4):455-463. doi:10.1111/jce.12608

21. Nagy-Baló E, Martirosyan M, Sándorfi G, et al. Cerebral micro-embolization during pulmonary vein isolation: Relation to post-ablation silent cerebral ischemia. Cardiol J. 2017;24(3):234-241. doi:10.5603/CJ.a2017.0030

22. Guijian L, Wenqing Z, Xinggang W, et al. Association between ablation technology and asymptomatic cerebral injury following atrial fibrillation ablation. Pacing Clin Electrophysiol. 2014;37(10):1378-1391. doi:10.1111/pace.12432

23. Sauren LD, Van Belle Y, De Roy L, et al. Transcranial measurement of cerebral microembolic signals during endocardial pulmonary vein isolation: Comparison of three different ablation techniques. J Cardiovasc Electrophysiol. 2009;20(10):1102-1107. doi:10.1111/j.1540-8167.2009.01509.x

24. Hahne K, Mönnig G, Samol A. Atrial fibrillation and silent stroke: links, risks, and challenges. Vasc Health Risk Manag. 2016;12:65-74. doi:10.2147/VHRM.S81807

25. Martinek M, Sigmund E, Lemes C, et al. Asymptomatic cerebral lesions during pulmonary vein isolation under uninterrupted oral anticoagulation. EP Europace. 2013;15(3):325-331. doi:10.1093/europace/eus329

26. Buiatti A, von Olshausen $G$, Barthel P, et al. Cryoballoon vs. radiofrequency ablation for paroxysmal atrial fibrillation: an updated meta-analysis of randomized and observational studies. EP Europace. 2017;19(3):378-384. doi:10.1093/europace/euw262

27. Jiang J, Li J, Zhong G, et al. Efficacy and safety of the second-generation cryoballoons versus radiofrequency ablation for the treatment of paroxysmal atrial fibrillation: a systematic review and meta-analysis. J Interv Card Electrophysiol. 2016. doi:10.1007/s10840-016-0191-9

28. Lustgarten DL, Keane D, Ruskin J. Cryothermal ablation: Mechanism of tissue injury and current experience in the treatment of tachyarrhythmias. Prog Cardiovasc Dis. 1999;41(6):481-498. doi:10.1016/S0033-0620(99)70024-1

29. Kaszala K, Ellenbogen K a. Biophysics of the Second-Generation Cryoballoon: Cryobiology of the Big Freeze. Circ Arrhythmia Electrophysiol. 2015;8(1):15-17. doi:10.1161/CIRCEP.115.002675

30. Tang B. Impact of contact force technology on reducing the recurrence and major complications of atrial fibrillation ablation: A systematic review and meta-analysis. Anatol J Cardiol. 2017;17(2):82-91. doi:10.14744/AnatolJCardiol.2016.7512

31. Ullah W, McLean A, Tayebjee MH, et al. Randomized trial comparing pulmonary vein isolation using the SmartTouch catheter with or without real-time contact force data. Heart Rhythm. 2016;13(9):1761-1767. doi:10.1016/j.hrthm.2016.05.011 
32. Marijon E, Fazaa S, Narayanan K, et al. Real-time contact force sensing for pulmonary vein isolation in the setting of paroxysmal atrial fibrillation: Procedural and 1-year results. J Cardiovasc Electrophysiol. 2014;25(2):130-137. doi:10.1111/jce.12303

33. Kautzner J, Neuzil P, Lambert H, et al. EFFICAS II: Optimization of catheter contact force improves outcome of pulmonary vein isolation for paroxysmal atrial fibrillation. EP Europace. 2015;17(8):1229-1235. doi:10.1093/europace/euv057

34. Zhou X, LV W, Zhang W, et al. Comparative efficacy and safety of contact force-sensing catheter and second-generation cryoballoon ablation for atrial fibrillation: a meta-analysis. Brazilian J Med Biol Res. 2017;50(9):1-7. doi:10.1590/1414-431×20176409

35. Afzal MR, Samanta A, Reddy M, et al. Reply to the Editor- Clinical Benefit of Contact ForceSensing Catheters in Ablation of Atrial Fibrillation: Meta-analysis to Dig for Truth or Bias? Heart Rhythm. 2016;13(1):e1-e2. doi:10.1016/J.HRTHM.2015.10.035

36. Shurrab M, Di Biase L, Briceno DF, et al. Impact of Contact Force Technology on Atrial Fibrillation Ablation: A Meta-Analysis. J Am Heart Assoc. 2015;4(9). doi:10.1161/JAHA.115.002476

37. Yokoyama K, Nakagawa H, Wittkampf FHMM, et al. Comparison of electrode cooling between internal and open irrigation in radiofrequency ablation lesion depth and incidence of thrombus and steam pop. Circulation. 2006;113(1):11-19. doi:10.1161/CIRCULATIONAHA.105.540062

38. Oza SR, Hunter TD, Biviano AB, et al. Acute Safety of an Open-Irrigated Ablation Catheter with 56Hole Porous Tip for Radiofrequency Ablation of Paroxysmal Atrial Fibrillation: Analysis from 2 Observational Registry Studies. J Cardiovasc Electrophysiol. 2014;25:852-858. doi:10.1111/jce.12403

39. Theis $\mathrm{C}$, Rostock $\mathrm{T}$, Mollnau $\mathrm{H}$, et al. The incidence of audible steam pops is increased and unpredictable with the ThermoCool${ }^{\circledR}$ surround flow catheter during left atrial catheter ablation: A prospective observational study. J Cardiovasc Electrophysiol. 2015;26(9):956-962. doi:10.1111/jce.12721

40. Gonna H, Domenichini G, Zuberi Z, et al. Initial clinical results with the ThermoCool ${ }^{\circledR}$ SmartTouch ${ }^{\circledR}$ Surround Flow catheter. EP Europace. 2017;19(8):1317-1321. doi:10.1093/europace/euw177

41. Chinitz LA, Melby DP, Marchlinski FE, et al. Safety and efficiency of porous-tip contact-force catheter for drug-refractory symptomatic paroxysmal atrial fibrillation ablation: results from the SMART SF trial. EP Europace. 2018;20(FI_3):f392-f400. doi:10.1093/europace/eux264

42. Stabile G, Di Donna P, Schillaci V, et al. Safety and efficacy of pulmonary vein isolation using a surround flow catheter with contact force measurement capabilities: A multicenter registry. J Cardiovasc Electrophysiol. 2017;28(7):762-767. doi:10.1111/jce.13227

43. Erden I, Erden EÇ, Golcuk E, et al. Impact of transesophageal echocardiography during transseptal puncture on atrial fibrillation ablation. J Arrhythmia. 2016;32(3):170-175. doi:10.1016/j.joa.2015.12.005

44. Kumar S, Sparks PB, Kalman JM. Letter by Kumar et al Regarding Article, "Extensive Intramural Esophageal Hematoma After Transesophageal Echocardiography During Atrial Fibrillation Ablation." Circulation. 2016;133(15):e594-e594. doi:10.1161/CIRCULATIONAHA.115.020368

45. Hsu JC, Badhwar N, Gerstenfeld EP, et al. Randomized trial of conventional transseptal needle versus radiofrequency energy needle puncture for left atrial access (the TRAVERSE-LA study). J Am Heart Assoc. 2013;2(5):e000428. doi:10.1161/JAHA.113.000428

46. Jauvert G, Grimard C, Lazarus A, et al. Comparison of a Radiofrequency Powered Flexible Needle with a Classic Rigid Brockenbrough Needle for Transseptal Punctures in Terms of Safety and Efficacy. Heart Lung Circ. 2015;24(2):173-178. doi:10.1016/j.hlc.2014.07.073

47. Kistler PM, Rajappan K, Jahngir M, et al. The impact of CT image integration into an 
electroanatomic mapping system on clinical outcomes of catheter ablation of atrial fibrillation. J Cardiovasc Electrophysiol. 2006;17(10):1093-1101. doi:10.1111/j.1540-8167.2006.00594.x

48. Oude Velthuis B, Molenaar M, Reinhart Dorman HG, et al. Use of three-dimensional computed tomography overlay for real-time cryoballoon ablation in atrial fibrillation reduces radiation dose and contrast dye. Neth Heart J. 2017;25(6):388-393. doi:10.1007/s12471-017-0962-7

49. Güler E, Güler GB, Demir GG, et al. Effect of Pulmonary Vein Anatomy and Pulmonary Vein Diameters on Outcome of Cryoballoon Catheter Ablation for Atrial Fibrillation. Pacing Clin Electrophysiol. 2015;38(8):989-996. doi:10.1111/pace.12660

50. Ivarez M, Bertomeu-Gonzá lez V, Fe Arcocha M, et al. Nonfluoroscopic Catheter Ablation. Results From a Prospective Multicenter Registry. Rev Esp Cardiol Rev Esp Cardiol. 2017;7070(9). doi:10.1016/j.rec.2016.12.040

51. Kozluk E, Rodkiewicz D, Piątkowska A, et al. Safety and efficacy of cryoablation without the use of fluoroscopy. Cardiol J. 2017. doi:10.5603/CJ.a2017.0065

52. Eitel C, Hindricks G, Dagres N, et al. EnSite VelocityTM cardiac mapping system: A new platform for 3D mapping of cardiac arrhythmias. Expert Rev Med Devices. 2010;7(2):185-192. doi:10.1586/erd.10.1

53. Merino JL. Tools or Toys? The 20-Year Anniversary of the Nonfluoroscopic Mapping System Dilemma. Rev Española Cardiol (English Ed. 2017;70(9):690-693. doi:10.1016/j.rec.2017.05.012

54. Nakamura K, Naito S, Kaseno K, et al. Integration of intracardiac echocardiography and computed tomography during atrial fibrillation ablation: Combining ultrasound contours obtained from the right atrium and ventricular outflow tract. Int J Cardiol. 2017;228:677-686. doi:10.1016/j.ijcard.2016.11.189

55. Packer DL, Kowal RC, Wheelan KR, et al. Cryoballoon ablation of pulmonary veins for paroxysmal atrial fibrillation: first results of the North American Arctic Front (STOP AF) pivotal trial. J Am Coll Cardiol. 2013;61(16):1713-1723. doi:10.1016/j.jacc.2012.11.064

56. Verma N, Gillespie CT, Argento AC, et al. Bronchial effects of cryoballoon ablation for atrial fibrillation. Heart Rhythm. 2017;14(1):12-16. doi:10.1016/j.hrthm.2016.10.012

57. van Opstal JM, Timmermans C, Blaauw Y, et al. Bronchial erosion and hemoptysis after pulmonary vein isolation by cryoballoon ablation. Heart Rhythm. 2011;8(9):1459. doi:10.1016/j.hrthm.2010.06.024

58. Kuck K, Furnkranz A. Cryoballoon ablation of atrial fibrillation. J Cardiovasc Electrophysiol. 2010;21(12):1427-1431. doi:10.1111/j.1540-8167.2010.01944.x

59. Ciconte G, de Asmundis C, Sieira J, et al. Single 3-minute freeze for second-generation cryoballoon ablation: One-year follow-up after pulmonary vein isolation. Heart Rhythm. 2015;12(4):673-680. doi:10.1016/j.hrthm.2014.12.026

60. Molenaar M, Hesselink T, Stevenhagen Y, et al. Less than 2 min cryoballoon applications result in comparable acute and 6-months success for the treatment of atrial fibrillation but reduced incidence of phrenic nerve palsy: results of the 123-study. Eur Heart J. 2016;37(Abstract supplement):633.

61. Han HC, Ha FJ, Sanders P, et al. Atrioesophageal Fistula: Clinical Presentation, Procedural Characteristics, Diagnostic Investigations, and Treatment Outcomes. Circ Arrhythm Electrophysiol. 2017;10(11). doi:10.1161/CIRCEP.117.005579

62. Barbhaiya CR, Kumar S, Guo Y, et al. Global Survey of Esophageal Injury in Atrial Fibrillation Ablation. JACC Clin Electrophysiol. 2016;2(2):143-150. doi:10.1016/j.jacep.2015.10.013

63. Kapur S, Barbhaiya C, Deneke T, et al. Esophageal injury and atrioesophageal fistula caused by 
ablation for atrial fibrillation. Circulation. 2017;136(13):1247-1255.

doi:10.1161/CIRCULATIONAHA.117.025827

64. Jung HK, Choung RS, Locke GR, et al. The Incidence, Prevalence, and Outcomes of Patients With Gastroparesis in Olmsted County, Minnesota, From 1996 to 2006. Gastroenterology. 2009;136(4):1225-1233. doi:10.1053/j.gastro.2008.12.047

65. Aksu T, Güler TE, Boyzel S, et al. An Underestimated Complication of Atrial Fibrillation Ablation: Gastroparesis. Pacing Clin Electrophysiol. 2015;38(5):653-654. doi:10.1111/pace.12607

66. Miyazaki S, Taniguchi H, Kusa S, et al. Factors Associated With Periesophageal Vagal Nerve Injury After Pulmonary Vein Antrum Isolation. J Am Hear Assoc. 2014;3(October):e001209. doi:10.1161/jaha.114.001209

67. Kuck K, Brugada J, Albenque J-P. Cryoballoon or radiofrequency ablation for atrial fibrillation. N Engl J Med. 2016;375(11):1099-1101. doi:10.1056/NEJMc1609160

68. Guiot A, Savouré A, Godin B, et al. Collateral nervous damages after cryoballoon pulmonary vein isolation. J Cardiovasc Electrophysiol. 2012;23(4):346-351. doi:10.1111/j.1540-8167.2011.02219.x

69. Kawasaki R, Gauri A, Elmouchi D, et al. Atrioesophageal fistula complicating cryoballoon pulmonary vein isolation for paroxysmal atrial fibrillation. J Cardiovasc Electrophysiol. 2014;25(7):787-792. doi:10.1111/jce.12426

70. Metzner A, Burchard A, Wohlmuth P, et al. Increased incidence of esophageal thermal lesions using the second-generation 28-mm cryoballoon. Circ Arrhythm Electrophysiol. 2013;6(4):769-775. doi:10.1161/CIRCEP.113.000228

71. Fürnkranz A, Bordignon S, Schmidt B, et al. Luminal esophageal temperature predicts esophageal lesions after second-generation cryoballoon pulmonary vein isolation. Heart Rhythm. 2013;10(6):789-793. doi:10.1016/j.hrthm.2013.02.021

72. Halbfass $P$, Müller $P$, Nentwich $K$, et al. Incidence of asymptomatic oesophageal lesions after atrial fibrillation ablation using an oesophageal temperature probe with insulated thermocouples: a comparative controlled study. EP Europace. 2016;19(3):euw070. doi:10.1093/europace/euw070

73. Black-Maier E, Pokorney SD, Barnett AS, et al. Risk of atrioesophageal fistula formation with contact force-sensing catheters. Heart Rhythm. 2017;14(9):1328-1333. doi:10.1016/j.hrthm.2017.04.024

74. Müller P, Dietrich J-W, Halbfass P, et al. Higher incidence of esophageal lesions after ablation of atrial fibrillation related to the use of esophageal temperature probes. Heart Rhythm. 2015;12(7):1464-1469. doi:10.1016/j.hrthm.2015.04.005

75. Knecht S, Sticherling C, Reichlin T, et al. Reliability of luminal oesophageal temperature monitoring during radiofrequency ablation of atrial fibrillation: insights from probe visualization and oesophageal reconstruction using magnetic resonance imaging. EP Europace. 2016;24(1):euw129. doi:10.1093/europace/euw129

76. Tschabrunn CM, Silverstein J, Berzin T, et al. Comparison between single- and multi-sensor oesophageal temperature probes during atrial fibrillation ablation: thermodynamic characteristics. EP Europace. 2015;17(6):891-897. doi:10.1093/europace/euu356

77. Deiss S, Metzner A, Ouyang F, et al. Incidence of Significant Delayed Esophageal Temperature Drop After Cryoballoon-Based Pulmonary Vein Isolation. J Cardiovasc Electrophysiol. 2016;27(8):913-917. doi:10.1111/jce.13008

78. Fürnkranz A, Bordignon S, Böhmig M, et al. Reduced incidence of esophageal lesions by luminal esophageal temperature-guided second-generation cryoballoon ablation. Heart Rhythm. 2015;12(2):268-274. doi:10.1016/j.hrthm.2014.10.033 
79. Kiuchi K, Okajima K, Shimane A, et al. Impact of esophageal temperature monitoring guided atrial fibrillation ablation on preventing asymptomatic excessive transmural injury. J Arrhythmia. 2016;32(1):36-41. doi:10.1016/j.joa.2015.07.003

80. Kuwahara T, Takahashi A, Takahashi Y, et al. Clinical characteristics and management of periesophageal vagal nerve injury complicating left atrial ablation of atrial fibrillation: lessons from eleven cases. J Cardiovasc Electrophysiol. 2013;24(8):847-851. doi:10.1111/jce.12130

81. Molenaar M, Hesselink T, Bouman D, et al. High incidence of low oesophageal temperatures during 2nd generation cryoballoon therapy for atrial fibrillation: predictive value of pulmonary vein- oesophageal distance measured by CT scan. Eur Heart J. 2016;37(Abstract Supplement):1083.

82. Kuwahara T, Takahashi A, Okubo K, et al. Oesophageal cooling with ice water does not reduce the incidence of oesophageal lesions complicating catheter ablation of atrial fibrillation: Randomized controlled study. EP Europace. 2014;16(6):834-839. doi:10.1093/europace/eut368

83. Mateos JCP, Mateos EIP, Peña TCS, et al. Simplified method for esophagus protection during radiofrequency catheter ablation of atrial fibrillation--prospective study of 704 cases. Rev Bras Cir Cardiovasc. 2015;30(2):139-147. doi:10.5935/1678-9741.20150009

84. Zellerhoff S, Lenze F, Eckardt L. Prophylactic proton pump inhibition after atrial fibrillation ablation: is there any evidence? EP Europace. 2011;13(9):1219-1221. doi:10.1093/europace/eur139

85. Halbfass $\mathrm{P}$, Pavlov B, Müller $\mathrm{P}$, et al. Progression from Esophageal Thermal Asymptomatic Lesion to Perforation Complicating Atrial Fibrillation Ablation: A Single-Center Registry. Circ Arrhythmia Electrophysiol. 2017;10(8):1-11. doi:10.1161/CIRCEP.117.005233

86. Kowalski M, Ellenbogen KA, Koneru JN. Prevention of phrenic nerve injury during interventional electrophysiologic procedures. Heart Rhythm. 2014;11(10):1839-1844. doi:10.1016/j.hrthm.2014.06.019

87. Ho SY, Cabrera JA, Sanchez-Quintana D. Left atrial anatomy revisited. Circ Arrhythmia Electrophysiol. 2012;5(1):220-228. doi:10.1161/CIRCEP.111.962720

88. Santangeli P, Marchlinski FE. Left Phrenic Nerve Pacing From the Left Subclavian Vein: Novel Method to Monitor for Left Phrenic Nerve Injury During Catheter Ablation. Circ Arrhythmia Electrophysiol. 2015;8(1):241-242. doi:10.1161/CIRCEP.114.002302

89. Ghosh J, Singarayar S, Kabunga P, et al. Subclavian vein pacing and venous pressure waveform measurement for phrenic nerve monitoring during cryoballoon ablation of atrial fibrillation. EP Europace. 2015;17(6):884-890. doi:10.1093/europace/euu341

90. Ghosh J, Sepahpour A, Chan KH, et al. Immediate balloon deflation for prevention of persistent phrenic nerve palsy during pulmonary vein isolation by balloon cryoablation. Heart Rhythm. 2013;10(5):646-652. doi:10.1016/j.hrthm.2013.01.011

91. Franceschi F, Dubuc M, Guerra PG, et al. Phrenic nerve monitoring with diaphragmatic electromyography during cryoballoon ablation for atrial fibrillation: The first human application. Heart Rhythm. 2011;8(7):1068-1071. doi:10.1016/j.hrthm.2011.01.047

92. Franceschi F, Dubuc M, Guerra PG, et al. Diaphragmatic electromyography during cryoballoon ablation: A novel concept in the prevention of phrenic nerve palsy. Heart Rhythm. 2011;8(6):885891. doi:10.1016/j.hrthm.2011.01.031

93. Andrade JG, Dubuc M, Ferreira J, et al. Histopathology of cryoballoon ablation-induced phrenic nerve injury. J Cardiovasc Electrophysiol. 2014;25(2):187-194. doi:10.1111/jce.12296

94. Franceschi F, Koutbi L, Mancini J, et al. Novel electromyographic monitoring technique for prevention of right phrenic nerve palsy during cryoballoon ablation. Circ Arrhythmia Electrophysiol. 2013;6(6):1109-1114. doi:10.1161/CIRCEP.113.000517

95. Lakhani M, Saiful F, Parikh V, et al. Recordings of diaphragmatic electromyograms during 
cryoballoon ablation for atrial fibrillation accurately predict phrenic nerve injury. Heart Rhythm. 2014;11(3):369-374. doi:10.1016/j.hrthm.2013.11.015

96. Mondésert B, Andrade JG, Khairy P, et al. Clinical experience with a novel electromyographic approach to preventing phrenic nerve injury during cryoballoon ablation in atrial fibrillation. Circ Arrhythmia Electrophysiol. 2014;7(4):605-611. doi:10.1161/CIRCEP.113.001238

97. Okishige K, Aoyagi H, Kawaguchi N, et al. Novel method for earlier detection of phrenic nerve injury during cryoballoon applications for electrical isolation of pulmonary veins in patients with atrial fibrillation. Heart Rhythm. 2016;13(9):1810-1816. doi:10.1016/j.hrthm.2016.05.003 


\section{SHORTER CRYOBALLOON APPLICATIONS TIMES DO AFFECT EFFICACY BUT RESULT IN LESS PHRENIC NERVE INJURY: RESULTS OF THE RANDOMIZED 123 STUDY}

Marleen MD Molenaar, Carl C Timmermans, Tim Hesselink, Marcoen F Scholten, Rachel MA ter Bekke, Justin GLM Luermans, Marjolein Brusse-Keizer, Karin Kraaier, Bernard Ten Haken, Jan G Grandjean, Kevin Vernooy, Jurren M van Opstal

Pacing and Clinical Electrophysiology. 2019: 42 (5), 508-514

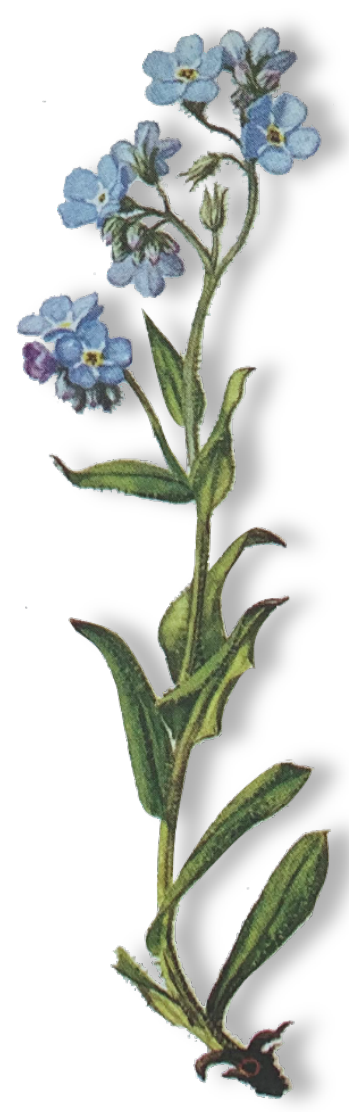




\section{Abstract}

Background The second-generation cryoballoon significantly improves outcome of pulmonary vein isolation (PVI) but may cause more complications than the firstgeneration. Currently, no consensus regarding optimal cryoballoon application time exists. The 123-study aimed to assess the minimal cryoballoon application duration necessary to achieve PVI (primary endpoint) and the effect of application duration on prevention of phrenic nerve injury (PNI).

Methods Patients $<75$ years of age with paroxysmal atrial fibrillation, normal PV anatomy, and left atrial size $<40 \mathrm{cc} / \mathrm{m}^{2}$ or $<50 \mathrm{~mm}$ were randomized to two applications of different duration: "short," "medium," or "long." A total of 222 patients were enrolled, 74 per group.

Results Duration per application was 105 (101-108), 164 (160-168), and 224 (219-226) S and isolation was achieved in 79,89, and 90\% $(P<0.001)$ of the PVs after two applications in groups short, medium, and long, respectively. Only for the left PVs, the success rate of the short group was significantly less compared to the mediumand long-duration groups $(P<0.001)$. PNI during the procedure occurred in $19 \mathrm{PVS}$ (6.5\%) in the medium and in $20 \mathrm{PVs}(6.8 \%)$ in the long duration groups compared to only five PVs $(1.7 \%)$ in the short duration group $(P<0.001)$.

Conclusions Short cryoballoon ablation application times, less than $2 \mathrm{~min}$, did affect the success for the left PVs but not for the right PVs and resulted in less PNI. A PV tailored approach with shorter application times for the right PVs might be advocated. 


\section{Background}

Since the observation of pulmonary vein (PV) ectopy as the origin for atrial fibrillation (AF), pulmonary vein isolation (PVI) has become the cornerstone for nonpharmaceutical AF treatment. Continuous improvement of ablation devices made catheter ablation the first-line therapy in selected patients, where radiofrequency (RF) and cryoballoon PVI are the recommended methods of the use'.

Cryoballoon ablation has shown to be safe and efficacy rates are non-inferior to RF ablation². The re-engineered second-generation cryoballoon (Arctic Front Advance, Medtronic Inc., Minneapolis, MN, USA) resulted in more homogeneous cooling, which improved the procedure efficacy ${ }^{3,4}$. However, this higher efficacy goes handin-hand with an increased risk of complications. In particular, when compared to the first-generation, the second-generation cryoballoon is associated with a higher incidence of right phrenic nerve injury (PNI), which remains a considerable drawback of this technique ${ }^{4-7}$.

Historically, 4-min applications with a bonus application after isolation have been the standard for the first-generation cryoballoon. With the aim to reduce complications without affecting treatment efficacy, the dosing of cryoenergy becomes of critical importance for the second-generation cryoballoon. So far protocols with shorter ablation times have shown encouraging results ${ }^{8-10}$. However, this has been only demonstrated in retrospect and at this moment, uniform consensus regarding the optimal cryoballoon application time is lacking.

The main objective of the present study was to prospectively assess, in a randomized fashion, the minimal cryoballoon application time necessary to achieve $\mathrm{PVI}$ and the effect of minimizing application duration on preventing PNI.

\section{Methods}

\section{Study design}

This clinical trial was designed as a patient-blinded, randomized, dual centre (Medisch Spectrum Twente [MST], Enschede and Maastricht University Medical Centre+ [MUMC], Maastricht, the Netherlands) study. The study was approved by both local institutional ethical committees on human research and was registered in the clinicaltrial.gov database (no. NCT02074566).

\section{Study population}

Patients fulfilling the following inclusion criteria were enrolled in the study: paroxysmal AF eligible for PVI according to the ESC guidelines and an age $<75$ 
years ${ }^{1,11}$. Patients were excluded if they had undergone a prior PVI; if the life expectancy was $<12$ months; if there was pregnancy at the time of the procedure, contrast allergy, creatinine clearance $<60 / \mathrm{mL} / \mathrm{min} / 1.73 \mathrm{~m}^{2}$, left ventricular ejection fraction (LVEF) $<40 \%$, left atrial (LA) volume $>40 \mathrm{cc} / \mathrm{m}^{2}$, or LA diameter $>50 \mathrm{~mm}$, or aberrant PV anatomy.

\section{Randomization}

After written informed consent, patients were randomized to "short," "medium," or "long" application duration protocols in a 1:1:1 fashion. Patients received two applications of 1 (short), 2 (medium), or 3 (long) min after reaching maximal $\mathrm{N}_{2} \mathrm{O}$ cooling flow; hence, this study was called "the 123-study." Maximal $\mathrm{N}_{2} \mathrm{O}$ flow is achieved at 72 Standard Cubic Centimeters Minute (SCCM) for the 28-mm balloon and 56 SCCM for the 23-mm balloon and the time to reach maximal flow may differ per application. The study flow chart is illustrated in Figure 1.

\section{PVI procedure}

The procedures were performed under conscious sedation at the MUMC site and under general anaesthesia, with continuous blood pressure monitoring, at the MST site. Heparin was administered to achieve an activated clotting time of $>300 \mathrm{~s}$ during the procedure. Pacing on the Achieve (Medtronic Inc.) mapping catheter in the left superior PV prior to cryotherapy was performed to exclude left-sided phrenic capture. During cryoablation of the right-sided PVs, the right phrenic nerve was continuously stimulated by pacing from the superior caval vein or the right subclavian vein. When diminished diaphragm excursion was noted during cryotherapy, the application was stopped immediately using the double stop technique $^{12}$. If a premature termination caused by diminished diaphragm excursion had to be performed, this was qualified as "PNI." At the end of a procedure in which PNI occurred, recovery of phrenic nerve function was tested by the stimulation.

Applications were performed using a double-walled cryoballoon (Arctic Front Advance [ $n=208]$ or Arctic Front Advanced ST [ $n=14]$, Medtronic Inc.). The preference was to use the 28-mm balloon. If the PV diameter was too small to occlude, we used the 23-mm balloon. Quality of PV occlusion was visualized by contrast administration during fluoroscopy and scored on a semiquantitative scale with grades 1 (very poor) to 4 (excellent), as described previously ${ }^{13}$. An occlusion of 4 was aimed for in every application. The operators were not blinded for the application duration. The second application started at least 1 min after deflation of the cryoballoon of the first application. The Achieve mapping catheter was inserted through the inner lumen of the balloon to assess PV signals before, during, and 


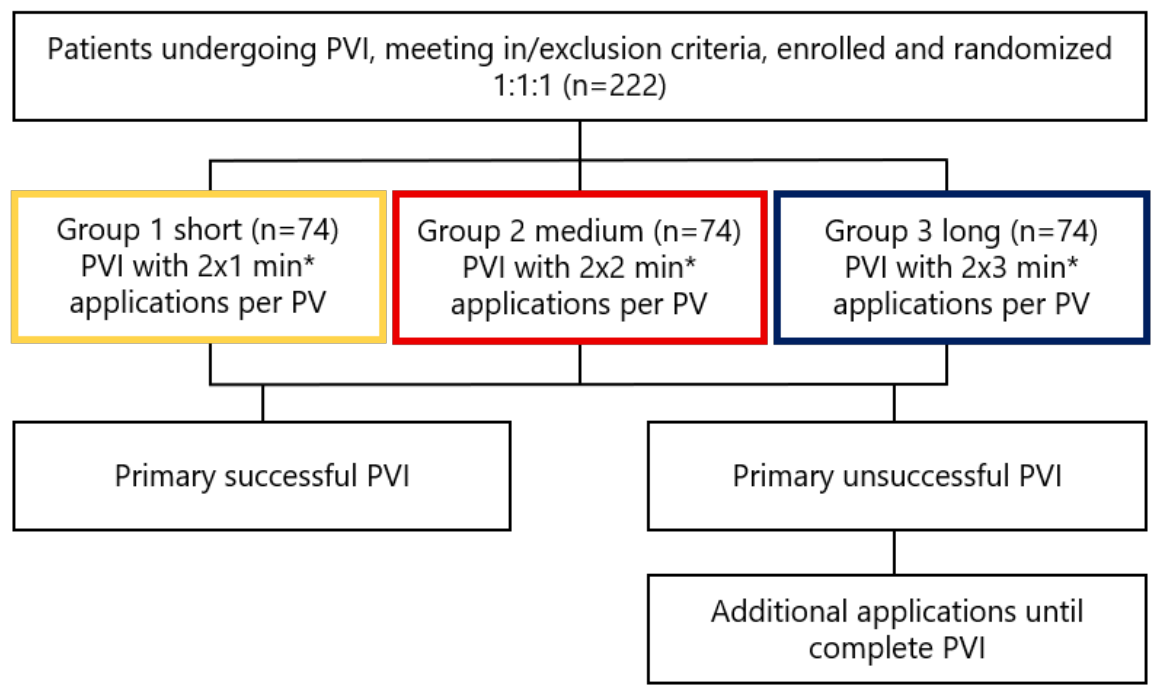

Figure 1 123-Study flowchart. PV: Pulmonary Vein, PVI: Pulmonary Vein Isolation *Time starts after maximal $\mathrm{N}_{2} \mathrm{O}$ flow is reached 
after every application and to guide the positioning of the balloon. The initial application sequence was left superior PV (LSPV), left inferior PV (LIPV), right superior PV (RSPV), and right inferior PV (RIPV) for the first 11 patients. The sequence was adjusted to LSPV, LIPV, RIPV, and RSPV after the occurrence of PNI in the RSPV in two patients, whereafter the RIPV could not be safely isolated anymore because the phrenic nerve could not be stimulated. Time to isolation (TTI), if visible, was measured for the last 170 patients.

Primary successful isolation was defined as an isolation of that particular PV, proven by an entrance and exit block, after two applications. Primary unsuccessful isolation was defined if there was no PV isolation after two applications. In PVs with primary unsuccessful isolation, supplementary applications were applied to ensure complete isolation for all the PVs. The number and duration of these supplementary application(s) was determined by the operating physician. In PVs, extremely challenging to isolate the Achieve catheter was placed in other PV branches of the same PV or the pull-down manoeuvre was used ${ }^{14}$.

\section{Statistical analysis}

The study was designed to assess three noninferiority hypotheses comparing the different groups (short, medium, long) independent of each other. Prior to the study, a noninferiority margin of $10 \%$ was determined and a power calculation that assumes success was performed. Based on this calculation, a total of 222 patients $(3 \times 74)$ were enrolled. Data analysis were performed using SPSS (version 22; IBM Corp., Armonk, NY, USA).

Continuous variables are summarized by mean \pm standard deviation for normally distributed variables or median with interquartile ranges for non-normally distributed variables. Categorical variables are expressed as numbers and percentages. Statistical differences for continuous normally distributed variables were analyzed using ANOVA for three groups. In case the analysis of variance (ANOVA) showed significant differences, post-hoc tests according to the method of Tukey's Honestly Significant Differences were performed. For non-normally distributed variables, the Kruskal-Wallis test was used to test for differences between the three groups with post-hoc Mann-Whitney $U$ tests using BonferroniHolm correction. Statistical differences between the groups for categorical variables were analyzed using Chi-square or Fisher exact tests, as appropriate. In case significant differences between categorical variables were observed, post-hoc tests according to Bonferroni-Holm were performed. Also, 95\% confidence intervals 
(Cls) to assess noninferiority for the success percentages were calculated using Epi Info.

All procedural characteristics are reported per patient, while success is mentioned per PV. Complication rates are reported per patient, as well as per PV. Balloon temperature, occlusion grade, thaw time, and application duration are reported per application.

\section{Results}

\section{Patient characteristics}

Between July 2014 and August 2017, a total of 222 patients, 74 patients in each group, were enrolled. As shown in Table 1, there were no significant differences between the baseline patient characteristics for the three groups.

\section{Procedural characteristics}

Table 2 shows the procedural characteristics for the three groups. As the consequence of the study design, the mean application duration was significantly different for the three groups. The time to reach maximal $\mathrm{N}_{2} \mathrm{O}$ cooling flow was 44 [40-48] $\mathrm{S}$ and did not differ between the groups ( $P=0.21$, known for all but 167 applications). The procedure time was significantly shorter in the short group when compared to the long group.

\section{PVI success}

Eight out of 888 PVs were not targeted for PVI with the cryoballoon. Applications could not be delivered due to technical malfunction of the cryo console $(n=2)$, the occurrence of PNI after ablation of the ipsilateral PV $(n=2)$, PV anatomy in which it appeared to be possible to occlude the ostium of the LSPV and LIPV simultaneously $(n=3)$, and impossibility to occlude the PV using the cryoballoon $(n=1)$.

The remaining 880 out of 888 PVs were targeted for PVI with the cryoballoon. The number of primary successful isolated PVs was significantly lower in the short group (79\% [Cl, 74-83\%]) when compared to the medium group (89\% [Cl, 85-92\%]) and the long group (90\% [Cl: 86-93\%]) (Figure 2), but did not exceed the predefined margin of $10 \%$ regarding the noninferiority analysis. A subanalysis, comparing the successes for the groups for the four different PVs, showed that the significant difference between the groups was only present for the left PVs and there was no significant difference in success between the groups for the right PVs (Figure 3). The success after a single application differed significantly between the short (62\%) 
Table 1 Baseline patient characteristics

\begin{tabular}{|c|c|c|c|c|c|}
\hline & $\begin{array}{l}\text { Total } \\
(n=222)\end{array}$ & $\begin{array}{l}\text { Short } \\
\text { group } \\
(n=74)\end{array}$ & $\begin{array}{l}\text { Medium } \\
\text { group } \\
(n=74)\end{array}$ & $\begin{array}{l}\text { Long } \\
\text { group } \\
(n=74)\end{array}$ & $P$-value \\
\hline Age (years) & $58 \pm 9$ & $58 \pm 10$ & $59 \pm 9$ & $57 \pm 9$ & 0.40 \\
\hline Gender (male,\%) & $143(64)$ & $46(62)$ & $47(64)$ & $50(68)$ & 0.78 \\
\hline $\mathrm{CHA}_{2} \mathrm{DS}_{2}$ VASC score & $1.2 \pm 1.1$ & $1.3 \pm 1.2$ & $1.2 \pm 1.2$ & $1.0 \pm 1.0$ & 0.34 \\
\hline Hypertension (\%) & $73(33)$ & $26(35)$ & $21(28)$ & $26(35)$ & 0.60 \\
\hline Diabetes mellitus 2 (\%) & $7(3)$ & $3(4)$ & $1(1)$ & $3(4)$ & 0.70 \\
\hline Prior revascularisation (\%)* & $7(3)$ & $3(4)$ & $3(4)$ & $3(4)$ & 1.00 \\
\hline EHRA class & $2.4 \pm 0.5$ & $2.4 \pm 0.5$ & $2.4 \pm 0.5$ & $2.4 \pm 0.6$ & 0.90 \\
\hline Body mass index $\left(\mathrm{kg} / \mathrm{m}^{2}\right)$ & $27 \pm 3$ & $27 \pm 3$ & $27 \pm 3$ & $26 \pm 3$ & 0.48 \\
\hline Left atrial volume $\left(\mathrm{cc} / \mathrm{m}^{2}\right)^{+}$ & $30 \pm 8$ & $29 \pm 8$ & $29 \pm 10$ & $30 \pm 8$ & 0.72 \\
\hline
\end{tabular}

Data are expressed as mean \pm SD or $n(\%)$, unless otherwise indicated.

*Revascularisation by percutaneous coronary intervention and/or coronary artery bypass grafting.

+Left atrial volume in $\mathrm{cc} / \mathrm{m}^{2}$ was not available in 16 patients due to a limited echocardiographic window or missing echocardiography recordings. In these patients left atrial volume in $\mathrm{mm}$ was available $(37 \pm 6 \mathrm{~mm})$

Table 2 Procedural characteristics

\begin{tabular}{|c|c|c|c|c|c|}
\hline & $\begin{array}{l}\text { Total } \\
(n=222)\end{array}$ & $\begin{array}{l}\text { Short } \\
\text { group } \\
(n=74)\end{array}$ & $\begin{array}{l}\text { Medium } \\
\text { group } \\
(n=74)\end{array}$ & $\begin{array}{l}\text { Long } \\
\text { group } \\
(\mathrm{n}=74)\end{array}$ & $P$-value \\
\hline $\begin{array}{l}\text { Duration per application (s) } \\
\text { (median [IQR]) }\end{array}$ & 163 [107-219] & 105 [101-108] & 164 [160-168] & 224 [219-226] & $<0.001^{1}$ \\
\hline $\begin{array}{l}\text { Lowest balloon temperature } \\
\text { per application }(\circ \mathrm{C})\end{array}$ & $-47 \pm 8$ & $-45 \pm 8$ & $-47 \pm 8$ & $-48 \pm 7$ & $<0.001^{2}$ \\
\hline $\begin{array}{l}\text { Thaw time } \\
\text { per application (s) }\end{array}$ & $9 \pm 5$ & $8 \pm 4$ & $9 \pm 5$ & $9 \pm 5$ & $<0.001^{3}$ \\
\hline $23 \mathrm{~mm}(\%)$ & $5(2.3)$ & $1(1.4)$ & $2(2.7)$ & $2(2.7)$ & 0.52 \\
\hline $28 \mathrm{~mm}(\%)$ & $212(96)$ & $70(95)$ & $70(95)$ & $72(97)$ & \\
\hline $23+28 \mathrm{~mm}(\%)$ & $5(2.3)$ & $3(4.1)$ & $2(2.7)$ & $0(0)$ & \\
\hline Procedure time (hh:mm) & $1: 28 \pm 0: 24$ & $1: 22 \pm 0: 23$ & $1: 29 \pm 0: 27$ & $1: 32 \pm 0: 20$ & $0.04^{4}$ \\
\hline Contrast (mL) & $67 \pm 38$ & $69 \pm 36$ & $70 \pm 44$ & $63 \pm 32$ & 0.49 \\
\hline Radiation time (hh:mm) & $0: 22 \pm 0: 10$ & $0: 22 \pm 0: 10$ & $0: 23 \pm 0: 11$ & $0: 22 \pm 0: 10$ & 0.65 \\
\hline $\begin{array}{l}\text { Radiation Dose Area Product } \\
\left(\mathrm{mGy} \mathrm{cm}^{2}\right) \text { (median [IQR]) }\end{array}$ & $14.8[8.5-22.4]$ & $13.9[7.3-19.7]$ & $13.7[9.1-22.5]$ & $15.2[9.8-23.9]$ & 0.26 \\
\hline
\end{tabular}

Data are expressed in mean \pm SD or $n(\%)$ unless stated otherwise.

1 Significant between all groups.

${ }^{2}$ Significant between short \& medium and short \& long group

${ }^{3}$ Significant between short \& medium and short \& long group. Known for all but 300 applications.

${ }^{4}$ Significant between short and long group 


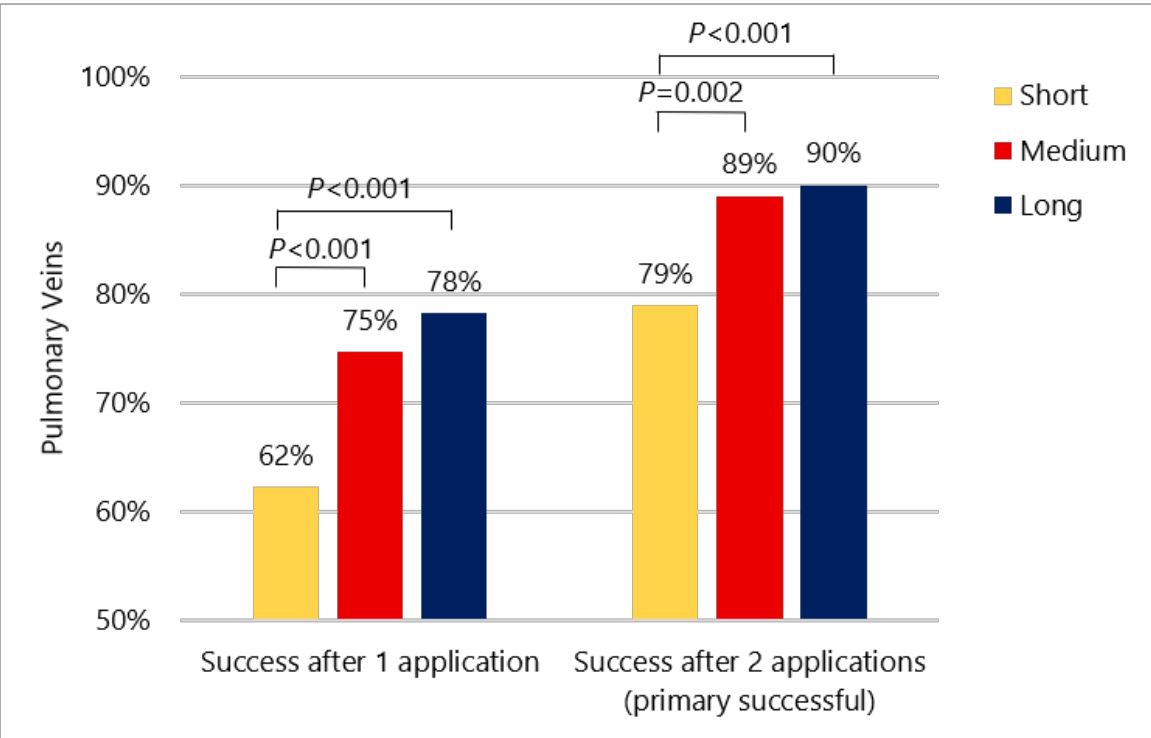

Figure 2 Percentage of successfully isolated pulmonary veins after 1 and 2 application(s)

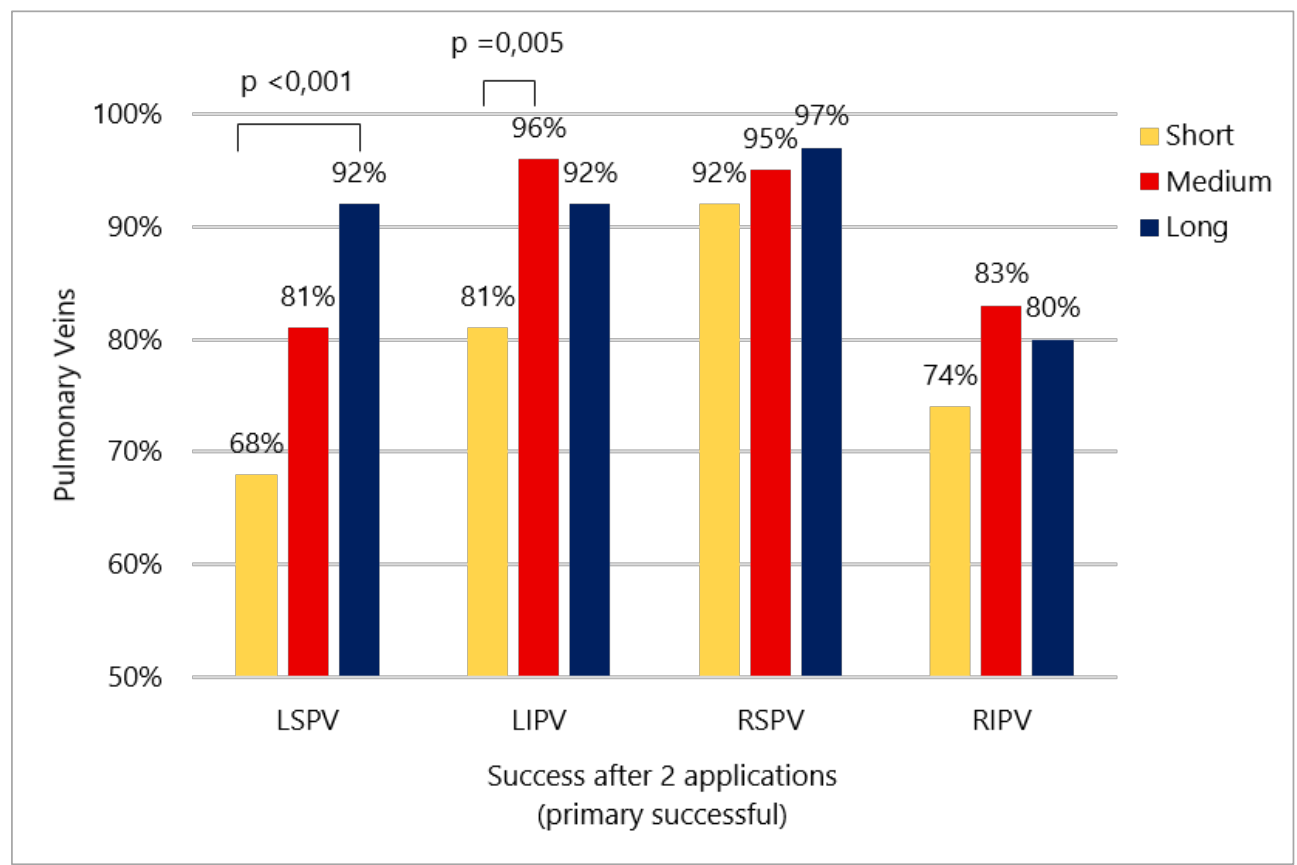

Figure 3 Percentage of successfully isolated pulmonary veins after 2 applications per PV. LIPV = left inferior PV; LSPV = left superior PV; PV = pulmonary vein; RIPV = right inferior PV; RSPV = 
and medium (75\%) groups and between the short and long (78\%) groups. An occlusion grade of $4 / 4$ was achieved in $77 \%$ of all applications. Occlusion grades of 3,2 , and $1 / 4$ were achieved in $15,8 \%$, and $<1 \%$, respectively, of all applications and occlusion grades did not differ significantly between the three groups $(P=0.71)$. The RIPV and LSPV were the most difficult PVs to isolate.

The number of supplementary cryoballoon applications per PV, needed to achieve complete PVI when isolation was primary unsuccessful, were $1.6 \pm 1.1$ for group 1 , $1.6 \pm 1.3$ for group 2, and $1.8 \pm 2.1$ for group 3 and did not differ between the groups $(P=0.87)$.

In six of 880 PVs (six patients) complete isolation could not be achieved with the cryoballoon due to the occurrence of PNI $(n=3)$ or due to a PV anatomy, which only allowed poor occlusion $(n=3)$. In two patients with a PV anatomy, which only allowed poor occlusion, isolation was ultimately achieved using point-by-point cryo or RF ablation. Therefore, eventually in all but four patients a successful PVI was performed.

\section{PNI}

In 39 of 222 patients (18\%), PNI occurred (44/880 PVs [5\%]). PNI occurred significantly more in the medium group ( $n=19 \mathrm{PVs}[6.5 \%])$ and in the long group ( $n=20 \mathrm{PVs}$ [6.8\%]) when compared to the short group $(n=5 \mathrm{PVs}[1.7 \%] P=0.006)$ (Figure 4). PNI did also differ significantly between the four PVs $(P<0.001)$ and only occurred in the RIPV $(n=10$ [1.1\%]) and RSPV $(n=34$ [3.9\%]). There was no significant difference in minimal balloon temperature in the right PV applications in which PNI occurred when compared to the right PV applications in which PNI did not occur $(P=0.71)$. In 27 of 39 (69\%) patients with PNI, phrenic nerve function had recovered completely at the end of the procedure.

\section{Other complications}

Three patients (one patient in every group) had vascular complications, all treated conservatively. One patient (medium group) experienced a minor transient ischemic attack. In one patient (medium group), ventricular fibrillation occurred during cryotherapy delivery in the RIPV with ST-segment elevation. A coronary angiogram showed no coronary artery occlusion, the ST-segment elevation resolved spontaneously, and the patient was discharged without sequela. No other major procedural complications occurred. 


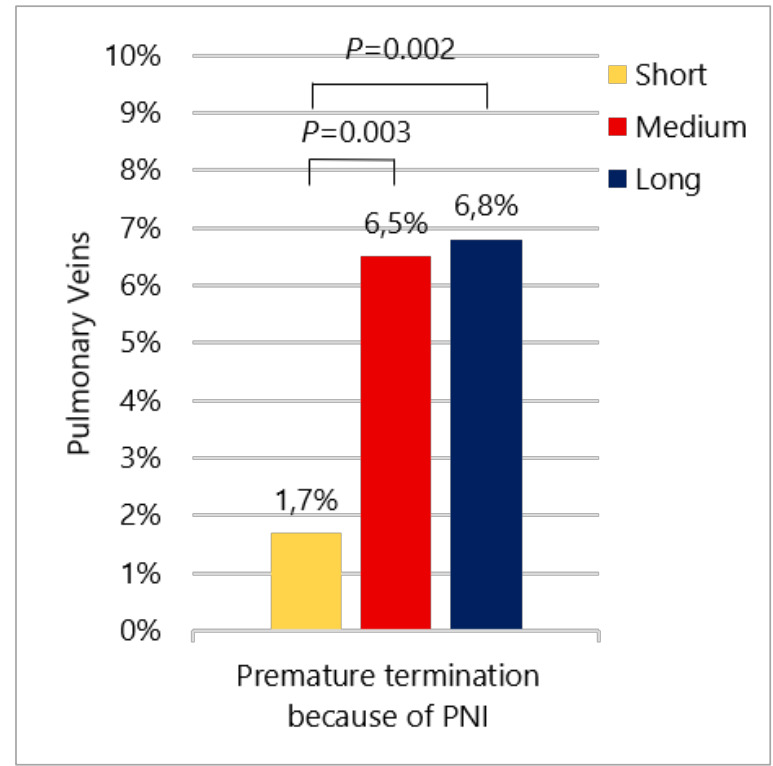

Figure 4 Percentage of pulmonary veins in which applications were prematurely terminated because of phrenic nerve injury (PNI) 


\section{Time to isolation}

In 670 applications in which isolation was achieved, an attempt to measure TTI was made. The TTI could be assessed in 316 of 670 (47\%). Mean TTI was shorter in PVs with persisting isolation at the end of the procedure compared to PVs showing early reconduction during the procedure ( $48 \pm 30 \mathrm{~s}$ vs $62 \pm 38 \mathrm{~s} ; P=0.006$ ). Mean balloon temperature at the time of isolation was $35 \pm 9^{\circ} \mathrm{C}$ and did not differ between these two groups $(P=0.31)$.

\section{Discussion}

The 123-study was designed to assess the minimal cryoballoon application duration for PVI while improving the safety. Patients were randomized to three different groups comparing standard to reduced application durations. The ultimate goal was to find an optimal freezing duration preserving the efficacy and improving the safety by avoiding PNI.

This prospective randomized study shows that (1) there is no difference in isolation rate between two applications of medium or long duration but two applications of short duration results in significantly less isolation, albeit that there is no proven superiority. (2) The significant differences in isolation rate between the three groups exist only for the left but not for the right PVs. (3) PNI occurred significantly more in the groups with medium and long freeze durations.

Historically, 4-minute applications with a bonus application after isolation were the standard for the first-generation cryoballoon ${ }^{15}$. With the advent of the secondgeneration cryoballoon, efficacy increased but it caused a decrease in safety, especially for right $\mathrm{PN}^{3,4}$. As a result, the paradigm shifted from efficacy to safety and, in recent years, the use of single applications and applications without bonus applications after successful isolation has been explored ${ }^{8,16-19}$. The omission of bonus applications did not compromise the efficacy of PVI and, depending on the $\mathrm{PV}$, the mean number of applications needed for isolation was found to be between 1 and $2^{8,17,19,20}$. Also, shorter application durations have been suggested and adopted, shortening the standard application duration in many centres to $180 \mathrm{~s}^{8,16}$, recently followed by studies that include TTI of a PV, if recordable during ablation ${ }^{10,21-}$ ${ }^{23}$. However, despite the encouraging results in terms of efficacy no previous study was performed prospectively in a randomized fashion comparing the standard to new dosing protocols. 


\section{Efficacy}

To eliminate the difference in effective ablation time within the three groups, due to differences in time needed to reach maximal $\mathrm{N}_{2} \mathrm{O}$ freezing flow, application durations for the three groups were defined as 1, 2, and 3 min after reaching maximal $\mathrm{N}_{2} \mathrm{O}$ flow. This resulted in a mean application duration of $104 \pm 13 \mathrm{~s}$ for the short group, $159 \pm 19 \mathrm{~s}$ for the medium group, and $212 \pm 35 \mathrm{~s}$ for the long group. Therefore, the long group may be compared to the historical standard of 4-min (240 s) applications.

The present study demonstrates that $2 \times 2$ min of ablation after reaching maximal $\mathrm{N}_{2} \mathrm{O}$ freezing flow does not impair the efficacy. Further shortening of the application duration significantly reduced results for the left PVs, although no inferiority was proven. In contrast, for the right PVs, even $2 \times 1$ min of freezing after reaching maximal $\mathrm{N}_{2} \mathrm{O}$ freezing flow showed similar isolation rates compared to longer cryo application durations.

The difference between the left and right PVs may be explained by the left lateral ridge, a fold of the atrial wall protruding into the endocardial LA surface between the orifices of the left PVs, and the ostium of the left atrial appendage characterized by thick muscular tissue and known to be associated with isolation gaps $^{24,25}$. Because of its thickness, it may take longer for cryoenergy to disperse and form a transmural lesion, therefore, a longer ablation duration may be needed to achieve isolation in the left PVs.

In this study, the number of successfully isolated PVs after a single application is similar to earlier reports using 180 and $240 \mathrm{~s}$ application durations $\mathrm{s}^{8,16,18-20}$.

\section{Safety}

In general, the complication rate in this study was similar to comparable previous studies. In our study, premature termination because of PNI occurred in 5\% of the PVs and was limited to the right PVs. Also, the RIPV accounted for a substantial amount of these premature terminations. Compared to the short group, premature termination because of $\mathrm{PNI}$ was more common in the groups with longer application durations.

The incidence of PNI has been reported to increase from $2-11 \%$ in the firstgeneration balloon ${ }^{4,15,26,27}$ to $3-24 \%$ in the second-generation balloon ${ }^{4,26-28}$. Previous study showed that the mean time to PNI was $123.6 \pm 47 \mathrm{~s}$ (median: 131), indicating that shorter application duration might prevent the occurrence of this complication $^{28}$. 
This study showed that shortening application duration reduces the incidence of PNI while, for the right PVs, even the ablation protocol with the shortest duration did not impair efficacy. Therefore, a PV-tailored approach with shorter application duration for the right PVs may be recommended. This is especially important as deterioration of diaphragm contractions sometimes already indicates irreversible or long-lasting nerve damage.

\section{TTI}

Most recent studies considering dosing protocols focus on the TTI of a $\mathrm{PV}^{10,21,23}$. The TTI in these protocols is used as a surrogate parameter for monitoring tissue physiology. This method has shown to be a predictor for durable lesions and enables a PV- and patient-tailored approach. In line with previous studies, we found a significantly longer TTI in the applications that showed reconduction during the procedure. However, not in every single application is TTI measurable. Therefore, an optimal ablation duration irrespective of TTI is needed. Furthermore, when incorporating TTI as the new standard in ablation protocols, data are needed to determine the optimal duration of an application àfter TTI is reached. Moreover, as recently mentioned, TTI as well as application time are both surrogate parameters ${ }^{29}$. Ideally, a biophysical parameter that directly measures ice formation or, ultimately, irreversible myocardial tissue deconstruction, should be available. Recently, the results of a first promising biophysical parameter that measures ice formation have been presented. This study is a proof of concept performed in an animal model, so we have to wait for clinical applicability ${ }^{30}$. 


\section{Conclusion}

A shortened application duration protocol of medium duration does not impair isolation success compared to the historical standard of long application duration. Further reduction to short application times did significantly impair isolation for the left, but not for the right, PVs. For the right PVs reduction to short application times, less than 2 min, resulted in less PNI.

The 123-study is a randomized study demonstrating that PNI during cryoballoon PVI can be reduced by further shortening the application duration for the right PVs, while maintaining similar efficacy rates. A PV-tailored approach with shorter application times for the right PVs might be advocated. 


\section{References}

1. Kirchhof P, Benussi S, Kotecha D, et al. 2016 ESC Guidelines for the management of atrial fibrillation developed in collaboration with EACTS. Eur Heart J. 2016;50(5):e1-e88.

doi:10.1093/ejcts/ezw313

2. Kuck K-H, Brugada J, Fürnkranz A, et al. Cryoballoon or Radiofrequency Ablation for Paroxysmal Atrial Fibrillation. N Engl J Med. 2016;374(23):2235-2245. doi:10.1056/NEJMoa1602014

3. Liu J, Kaufmann J, Kriatselis C, et al. Second generation of cryoballoons can improve efficiency of cryoablation for atrial fibrillation. Pacing Clin Electrophysiol. 2015;38(1):129-135. doi:10.1111/pace.12538

4. Aytemir K, Gurses KM, Yalcin MU, et al. Safety and efficacy outcomes in patients undergoing pulmonary vein isolation with second-generation cryoballoon. EP Europace. 2014;17(3):379-387. doi:10.1093/europace/euu273

5. Fürnkranz A, Bordignon S, Dugo D, et al. Improved 1-year clinical success rate of pulmonary vein isolation with the second-generation cryoballoon in patients with paroxysmal atrial fibrillation. J Cardiovasc Electrophysiol. 2014;25(8):840-844. doi:10.1111/jce.12417

6. Guiot A, Savouré A, Godin B, et al. Collateral nervous damages after cryoballoon pulmonary vein isolation. J Cardiovasc Electrophysiol. 2012;23(4):346-351. doi:10.1111/j.1540-8167.2011.02219.x

7. Fürnkranz A, Bordignon S, Schmidt B, et al. Luminal esophageal temperature predicts esophageal lesions after second-generation cryoballoon pulmonary vein isolation. Heart Rhythm. 2013;10(6):789-793. doi:10.1016/j.hrthm.2013.02.021

8. Ciconte G, de Asmundis C, Sieira J, et al. Single 3-minute freeze for second-generation cryoballoon ablation: One-year follow-up after pulmonary vein isolation. Heart Rhythm. 2015;12(4):673-680. doi:10.1016/j.hrthm.2014.12.026

9. Miyazaki S, Nakamura H, Taniguchi H, et al. Gastric hypomotility after second-generation cryoballoon ablation-Unrecognized silent nerve injury after cryoballoon ablation. Heart Rhythm. 2017;14(5):670-677. doi:10.1016/j.hrthm.2017.01.028

10. Chun KRJ, Stich M, Fürnkranz A, et al. Individualized cryoballoon energy pulmonary vein isolation guided by real-time pulmonary vein recordings, the randomized ICE-T trial. Heart Rhythm. 2017;14(4):495-500. doi:10.1016/j.hrthm.2016.12.014

11. John Camm A, Lip GYH, De Caterina R, et al. 2012 focused update of the ESC Guidelines for the management of atrial fibrillation. Eur Heart J. 2012;33(21):2719-2747. doi:10.1093/eurheartj/ehs253

12. Ghosh J, Sepahpour A, Chan KH, et al. Immediate balloon deflation for prevention of persistent phrenic nerve palsy during pulmonary vein isolation by balloon cryoablation. Heart Rhythm. 2013;10(5):646-652. doi:10.1016/j.hrthm.2013.01.011

13. Neumann T, Vogt J, Schumacher B, et al. Circumferential Pulmonary Vein Isolation With the Cryoballoon Technique. Results From a Prospective 3-Center Study. J Am Coll Cardiol. 2008;52(4):273-278. doi:10.1016/j.jacc.2008.04.021

14. Chun KRJ, Schmidt B, Metzner A, et al. The "single big cryoballoon" technique for acute pulmonary vein isolation in patients with paroxysmal atrial fibrillation: A prospective observational single centre study. Eur Heart J. 2009;30(6):699-709. doi:10.1093/eurheartj/ehn570

15. Packer DL, Kowal RC, Wheelan KR, et al. Cryoballoon ablation of pulmonary veins for paroxysmal atrial fibrillation: first results of the North American Arctic Front (STOP AF) pivotal trial. J Am Coll Cardiol. 2013;61(16):1713-1723. doi:10.1016/j.jacc.2012.11.064

16. Miyazaki S, Hachiya $\mathrm{H}$, Nakamura $\mathrm{H}$, et al. Pulmonary vein isolation using a second-generation cryoballoon in patients with paroxysmal atrial fibrillation: One-year outcome using a single big- 
balloon 3-minute freeze technique. J Cardiovasc Electrophysiol. 2016;27(12):1375-1380. doi:10.1111/jce.13078

17. Heeger C-H, Wissner E, Wohlmuth P, et al. Bonus-freeze: benefit or risk? Two-year outcome and procedural comparison of a "bonus-freeze" and "no bonus-freeze" protocol using the secondgeneration cryoballoon for pulmonary vein isolation. Clin Res Cardiol. 2016;105(9):774-782. doi:10.1007/s00392-016-0987-8

18. De Regibus V, lacopino S, Abugattas JP, et al. Single freeze strategy with the second- generation cryballoon for atrial fibrillation: a multicenter international retrospective analysis in a large cohort of patients. J Interv Card Electrophysiol. 2017;49(2):173-180. doi:10.1007/s10840-017-0254-6

19. Tebbenjohanns J, Höfer C, Bergmann L, et al. Shortening of freezing cycles provides equal outcome to standard ablation procedure using second-generation $28 \mathrm{~mm}$ cryoballoon after 15month follow-up. Europace. 2016;18(2):206-210. doi:10.1093/europace/euv189

20. Wissner E, Heeger $\mathrm{C}-\mathrm{H}$, Grahn $\mathrm{H}$, et al. One-year clinical success of a "no-bonus" freeze protocol using the second-generation $28 \mathrm{~mm}$ cryoballoon for pulmonary vein isolation. EP Europace. 2015;17(8):1236-1240. doi:10.1093/europace/euv024

21. Aryana A, Kenigsberg DN, Kowalski M, et al. Verification of a novel atrial fibrillation cryoablation dosing algorithm guided by time-to-pulmonary vein isolation: Results from the Cryo-DOSING Study. Heart Rhythm. 2017;14(9):1319-1325. doi:10.1016/j.hrthm.2017.06.020

22. Rottner L, Fink T, Heeger C-H, et al. Is less more? Impact of different ablation protocols on periprocedural complications in second-generation cryoballoon based pulmonary vein isolation. EP Europace. July 2017. doi:10.1093/europace/eux219

23. Reissmann B, Wissner E, Deiss S, et al. First insights into cryoballoon-based pulmonary vein isolation taking the individual time-to-isolation into account. EP Europace. 2017;19(10):1676-1680. doi:10.1093/europace/euw233

24. Cabrera JA, Ho SY, Climent V, et al. The architecture of the left lateral atrial wall: a particular anatomic region with implications for ablation of atrial fibrillation. Eur Heart J. 2008;29(3):356362. doi:10.1093/eurheartj/ehm606

25. Sánchez-Quintana D, Doblado-Calatrava M, Cabrera JA, et al. Anatomical basis for the cardiac interventional electrophysiologist. Biomed Res Int. 2015;2015:1-24. doi:10.1155/2015/547364

26. Fürnkranz A, Bordignon S, Schmidt B, et al. Incidence and characteristics of phrenic nerve palsy following pulmonary vein isolation with the second-generation as compared with the firstgeneration cryoballoon in 360 consecutive patients. EP Europace. 2015;17(4):574-578. doi:10.1093/europace/euu320

27. Martins RP, Hamon D, Césari O, et al. Safety and efficacy of a second-generation cryoballoon in the ablation of paroxysmal atrial fibrillation. Heart Rhythm. 2014;11(3):386-393. doi:10.1016/j.hrthm.2014.01.002

28. Chierchia G-B, Mugnai G, Hunuk B, et al. Impact on clinical outcome of premature interruption of cryoenergy delivery due to phrenic nerve palsy during second generation cryoballoon ablation for paroxysmal atrial fibrillation. J Cardiovasc Electrophysiol. 2015;26(9):950-955. doi:10.1111/jce.12718

29. Avitall B. Letter to the Editor-In search of cryoablation dose. Heart Rhythm. September 2017. doi:10.1016/j.hrthm.2017.09.026

30. Avitall B, Kalinski A, Kocheril GS, et al. Characteristics of Ice Impedance Recorded from a Ring Electrode Placed at the Anterior Surface of the Cryoballoon: Novel Approach to Define Ice Formation and Pulmonary Vein Isolation. Circ Arrhythmia Electrophysiol. 2018;11(4):e005949. doi:10.1161/CIRCEP.117.005949 
CHAPTER 4

\section{CHAPTER 4}

CHAPTER 4

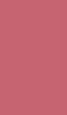




\section{SHORTER RIGHT SUPERIOR PULMONARY VEIN CRYOAPPLICATIONS RESULT IN LESS PHRENIC NERVE INJURY AND SIMILAR 1- YEAR FREEDOM FROM ATRIAL}

FIBRILLATION

Marleen MD Molenaar, Tim Hesselink, Rachel MA ter Bekke, Marcoen F Scholten, Randy Manusama, Laurent Pison, Marjolein Brusse-Keizer, Karin Kraaier, Bernard ten Haken, Jan G Grandjean, Carl C Timmermans, Jurren M van Opstal

Accepted (with revisions) for publication in Pacing and Clinical Electrophysiology

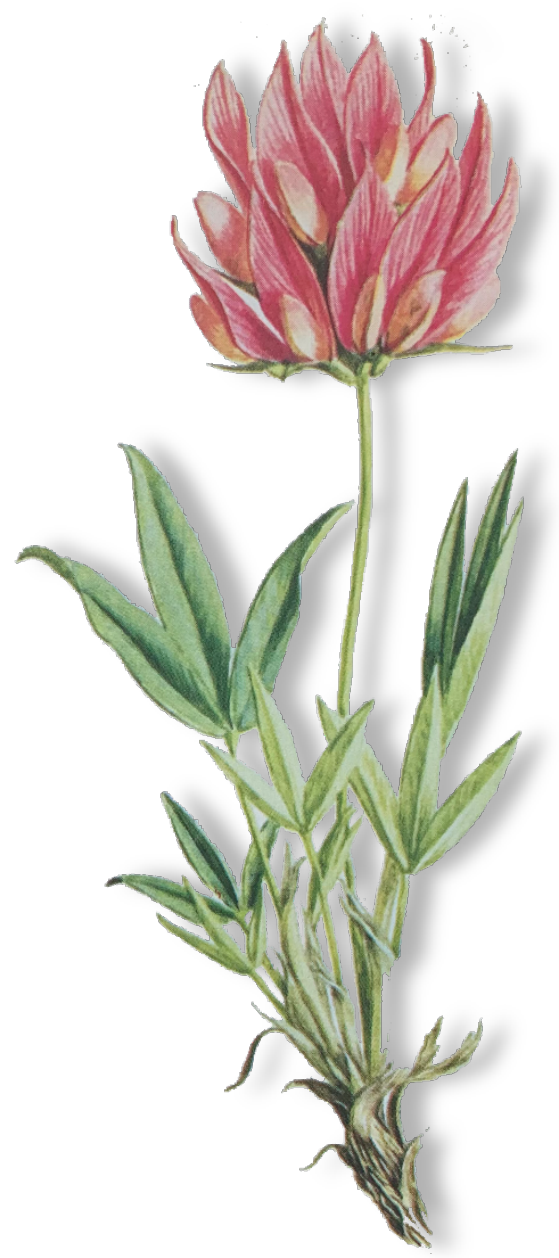




\section{Abstract}

Background In the 123-study we prospectively assessed, in a randomized fashion, the minimal cryoballoon application time necessary to achieve pulmonary vein (PV) isolation (PVI) in patients with paroxysmal atrial fibrillation (AF) with the aim to reduce complications by shortening the application duration. The first results of this study demonstrated that shortened cryoballoon applications ( $<2$ minutes) resulted in less phrenic nerve injury (PNI) without compromising acute isolation efficacy for the right PVs. We now report the 1-year follow-up results regarding safety and efficacy of shorter cryoballoon applications.

Methods 222 patients with AF were randomized to two applications of 1 min "short", 2 min "medium" or 3 min "long" duration, 74 per group. Recurrence of AF and PV reconduction at 1-year follow-up were assessed.

Results The overall 1-year freedom from AF was $79 \%$ and did not differ significantly between the short, medium and long application groups (77, 74 and $85 \%$ for short, medium and long application groups, respectively; $P=0.07$ ). randomization groups. In 30 patients a redo PVI procedure was performed. For all four PVs there was no significant difference in reconduction between the three groups. Reconduction was most common in the left superior PV (57\%). The right superior PV (RSPV) showed significantly less reconduction (17\%) compared to the other PVs.

Conclusions Shortening cryoballoon applications of the RSPV to $<2$ minutes results in less PNI while acute success and 1-year freedom from AF is not compromised. Therefore shorter cryoballoon applications (especially) in the RSPV could be used to reduce PNI. 


\section{Introduction}

Since the recognition that pulmonary vein (PV) ectopy can trigger atrial fibrillation $(\mathrm{AF})$, pulmonary vein isolation (PVI) has become the cornerstone for nonpharmaceutical treatment of AF. The continuous improvement of ablation techniques has made catheter ablation, including cryoablation, a first-line therapy worldwide ${ }^{1,2}$. When compared to radiofrequency (RF), cryoballoon based PVI is characterized by steeper learning curves, faster and more reproducible procedures and has documented non-inferiority in terms of safety and efficacy ${ }^{3}$. Cryoballoon has even been suggested to be the most advantageous choice in first-time PVI ${ }^{4}$.

Duration and dosing continue to be the topic of highest interest in cryoballoon PVI. Not only to achieve the best possible results in terms of efficacy but also in terms of safety. The optimal duration of cryoapplications is a ratio between acceptable efficacy and safety. Especially phrenic nerve injury (PNI) is a common complication and reducing phrenic nerve injury with cryoballoon PVI is of utmost importance ${ }^{5-8}$. In current clinical practice the duration of cryoapplications differs widely from 180 to 240 seconds $^{9,10}$.

In the 123-study ${ }^{11}$ we prospectively assessed, in a randomized fashion, the minimal cryoballoon application duration necessary to achieve PVI, with the aim to reduce procedural complications. The first results indicated that application duration reduction for the right PVs, less than two minutes, resulted in less PNI with similar acute isolation success ${ }^{11}$.

Here we present the 1-year follow-up results of the 123-study in terms of freedom from $\mathrm{AF}$ and the insights gained from redo procedures considering safety and efficacy.

\section{Methods}

The study design and the initial PVI procedure of the 123-study have been described in detail previously ${ }^{11}$. Patients received 2 applications of 1 (short), 2 (medium) or 3 (long) minutes after reaching maximal $\mathrm{N}_{2} \mathrm{O}$ cooling flow. 222 consecutive patients with paroxysmal AF were enrolled; 74 per group. Cryoballoon applications were performed using a double-walled cryoballoon (Arctic Front Advance ${ }^{\mathrm{TM}}(\mathrm{n}=208)$ or Arctic Front Advanced ST ${ }^{\mathrm{TM}}(\mathrm{n}=14)$, Medtronic Inc., MN, USA). Quality of PV occlusion was visualized by contrast administration during fluoroscopy and scored on a semiquantitative scale with grades 1 to 4 . An occlusion of 4 was aimed for in every application. The operators were not blinded for the application duration. 
The Achieve ${ }^{\mathrm{TM}}$ mapping catheter was inserted through the inner lumen of the balloon to assess PV signals before, during and after every application and to guide the positioning of the balloon. During cryoablation of the right-sided PVs, the right phrenic nerve was continuously stimulated by pacing from the superior caval vein or the right subclavian vein. In case of a diminished diaphragm excursion during cryotherapy, the application was stopped immediately using the double-stop technique $^{12}$. If a premature termination caused by diminished diaphragm excursion had to be performed, this was qualified as "PNI".

\section{Group definition}

Primary successful PV isolation was defined as isolation of that particular PV, proven by an entrance and exit block, after 2 applications. The primary success (PS) group represents the patients in which all PVs were successfully isolated after the designated application duration. Analysis of this group represents the success after $2 \times 1,2 \times 2$ or $2 \times 3$ minutes of ablation after reaching maximal $\mathrm{N}_{2} \mathrm{O}$ freezing flow.

The entire patient group can be seen as the intention-to treat-group. Representing a "real-world group" in which, depending on the randomization, a shortened application duration was used for the first two applications. If these applications were not sufficient for isolation, supplementary applications were applied to assure complete isolation for all the PVs. The number and duration of these supplementary application(s) were determined by the operating physician. The analysis of this group represents whether starting with shorter applications could be used to prevent complications reaching the same efficacy level.

\section{Follow-up}

All patients were scheduled for outpatient clinic visits at 3, 6 and 12 months post PVI. A 7-day holter ECG (Vitaphone $\mathrm{GmbH}$, Mannheim, Germany) capable of autodetecting AF or a $48 \mathrm{hr}$ regular holter ECG was recorded at 3, 6 and 12 months postPVI.

\section{Redo PVI procedure}

After confirmation of AF recurrence lasting $>30$ s, patients were offered a redo procedure. In the redo procedure, isolation was assessed and reconduction gaps were ablated using RF. Atrial geometry was constructed using either CARTO ${ }^{\circledR}$ (Biosense Webster, CA, USA) or NavX ${ }^{\mathrm{TM}}$ mapping systems (St Jude Medical, St Paul, MN, USA). Reconduction was assessed using a spiral catheter (Lasso ${ }^{\circledR}$, Biosensense Webster, CA, USA) for every PV. In PVs with identified reconduction, RF was applied until isolation was achieved. 


\section{Statistical Analysis}

Continuous variables are summarized by mean \pm standard deviation for normally distributed variables or median with interquartile ranges for non-normally distributed variables. Categorical variables are expressed as numbers and percentages. Statistical differences for continuous normally distributed variables were analyzed using ANOVA for three groups. In case the ANOVA showed significant differences, post hoc tests according to the method of Tukey's Honestly Significant Differences were performed. For non-normally distributed variables the KruskalWallis test was used to test for differences between the three groups with post hoc Mann Whitney U tests using Bonferroni-Holm correction. Statistical differences between the groups for categorical variables were analyzed using Chi-square or Fisher exact tests, as appropriate. In case significant differences between categorical variables were observed, post hoc tests according to Bonferroni-Holm were performed.

\section{Results}

Between July 2014 and August 2017 a total of 222 patients, 74 patients in each group, were enrolled and had follow-up until August 2018. There were no significant differences between the baseline patient characteristics for the 3 groups (Table 1) 1 . Significant differences in procedural characteristics were found for application duration and total procedure duration. Three patients were lost to follow-up. One patient withdrew consent, 2 patients died before reaching the 1-year follow-up point. One patient died 254 days post procedure of a metastasized sigmoid carcinoma and the other patient died 299 days post procedure without a specific cause of death found at autopsy. The former had symptomatic recurrence of AF during follow-up.

\section{Freedom from AF}

173 out of 220 (79\%) patients were free from recurrence of AF (Figure 1a). There was no significant difference between the three randomization groups for recurrence of $\operatorname{AF~}(P=0.07)$. In the initial PVI, all PVs were primarily successful (PS) isolated in $128 / 220$ (58\%) patients (Figure 1b). Differences in numbers of PS patients per randomization group were significant for short (45\%) vs medium (66\%) $(P=0.01)$ and short vs long (64\%) $(P=0.03)$. As shown in the first results, these differences were significant for the left PVs but not for the right PVs. The RSPV showed a PS percentage of $>90 \%$ for all randomization groups (Figure 3, Molenaar et al. 201911). The freedom from AF in the 128 PS patients was $84 \%$ and there was no significant 
Table 1 Patient and procedural characteristics

\begin{tabular}{llllll}
\hline & Total & $\begin{array}{l}\text { Short } \\
\text { group } \\
(\mathrm{n}=74)\end{array}$ & $\begin{array}{l}\text { Medium } \\
\text { group } \\
(\mathrm{n}=74)\end{array}$ & $\begin{array}{l}\text { Long } \\
\text { group } \\
(\mathrm{n}=74)\end{array}$ & P-value \\
\hline Age (years) & $58 \pm 9$ & $58 \pm 10$ & $59 \pm 9$ & $57 \pm 9$ & 0.40 \\
\hline Gender (male,\%) & $143(64)$ & $46(62)$ & $47(64)$ & $50(68)$ & 0.78 \\
\hline CHA2DS2VASC score & $1.2 \pm 1.1$ & $1.3 \pm 1.2$ & $1.2 \pm 1.2$ & $1.0 \pm 1.0$ & 0.34 \\
\hline EHRA class & $2.4 \pm 0.5$ & $2.4 \pm 0.5$ & $2.4 \pm 0.5$ & $2.4 \pm 0.6$ & 0.90 \\
\hline $\begin{array}{l}\text { Duration of 1 application (s) } \\
\text { (median [IQR]) }\end{array}$ & $163[107-219]$ & $105[101-108]$ & $164[160-168]$ & $224[219-226]$ & $<0.001^{1}$ \\
\hline Procedure time (hh:mm) & $1: 28 \pm 0: 24$ & $1: 22 \pm 0: 23$ & $1: 29 \pm 0: 27$ & $1: 32 \pm 0: 20$ & $0.04^{2}$ \\
\hline
\end{tabular}

Data are expressed in mean \pm SD or $n(\%)$ unless stated otherwise

${ }^{1}$ Significant between all groups

${ }^{2}$ Significant between short and long group

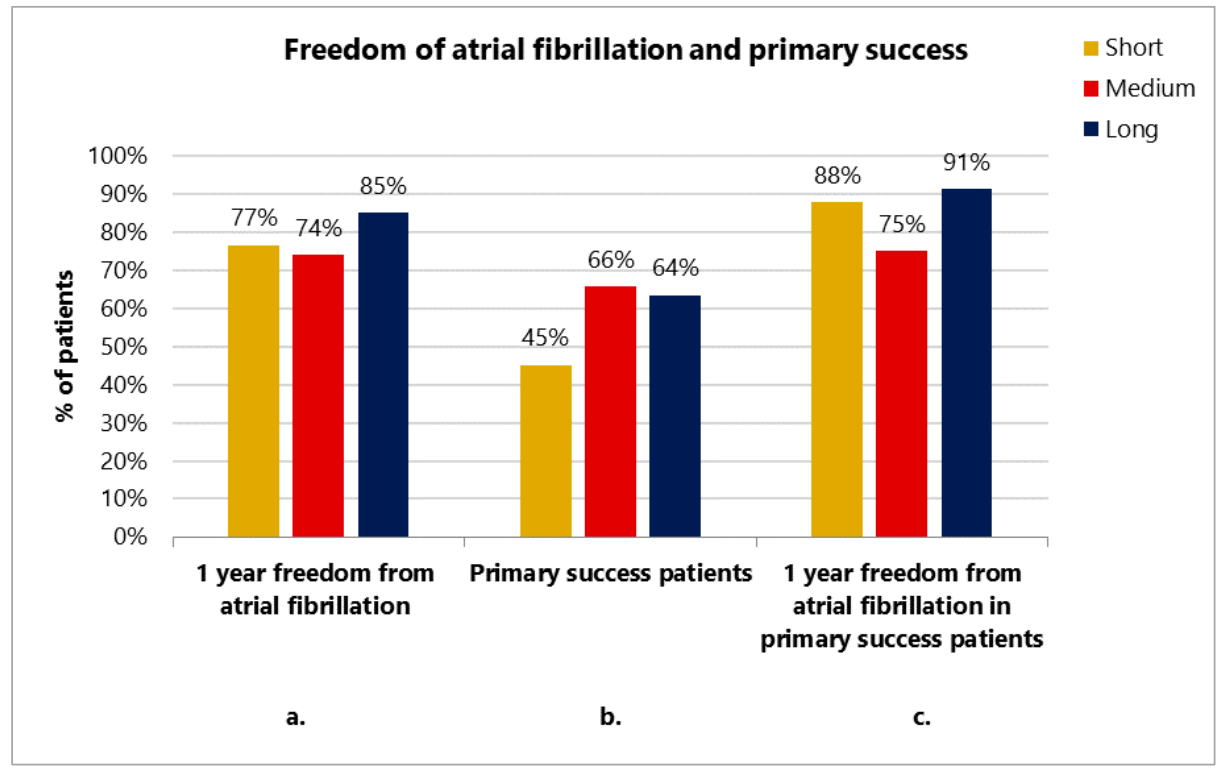

Figure 1 Distribution of patients over randomization-groups: a. 1-year freedom of atrial fibrillation (in all patients, intention to treat group) b. Primary success patients (= all PV's primarily successful isolated in initial procedure) c. 1-year freedom of atrial fibrillation in primary success patients 
difference ( $P=0.08$ ) between the randomization groups in the PS patients either (Figure 1c).

\section{Redo PVI procedure}

In 17/47 patients with recurrence of AF no redo procedure was performed. These patients experienced a significant reduction in AF burden following the initial PVI, with or without medical treatment, so that they renounced a redo procedure. In 30 patients, 12 in the short and medium group and 6 in the long group, a redo PVI procedure was performed. In 3 patients (10\%) all PVs showed durable isolations. Reconduction was found in one PV in 8 (27\%) patients, in two PVs in 13 (43\%) patients, in three PVs in 5 (17\%) patients and in one patient (3\%) all PVs showed reconduction.

For all PVs, as well as the subgroup of PVs in which isolation was primarily successful (PS PVS), most reconduction was found in the left superior PV (LSPV) (17/30 57\% all PV group, 15/25 60\% PS PV group). The RSPV (5/30, 17\%) showed significantly less reconduction compared to all other PVs (Figure 2 and 3, total-bar). A sub-analysis comparing reconduction in all four separate PVs showed no significant differences between the three randomization groups in the intention to treat PV group (Figure 2, short-medium-and-large bars) as well as the PS PV group (Figure 3, short-medium-and-large bars).

\section{Discussion}

The 123-study was designed to assess the minimal cryoballoon application duration for PVI while improving safety. Patients were randomized to 3 different groups comparing standard to reduced application durations, resulting in application durations of 105 [101-108] s for the short group, 164 [160-168] s for the medium group and 224 [219-226] s for the long group. The purpose of the study was to find an optimal freezing duration preserving efficacy and improving safety (especially) by avoiding PNI. The acute results of the 123-study demonstrated that the incidence of PNI was successfully reduced by using shorter cryoapplications.

The follow-up results show 1) similar 1-year freedom from AF for the short, medium and long duration groups in both the PS patients as well as the entire (intention to treat) patient group. Isolation assessment in redo procedures showed that 2) the RSPV had significantly less reconduction compared to all other PVs. 3) For all four PVs reconduction appeared independent of the previously applied duration of cryoapplications. 


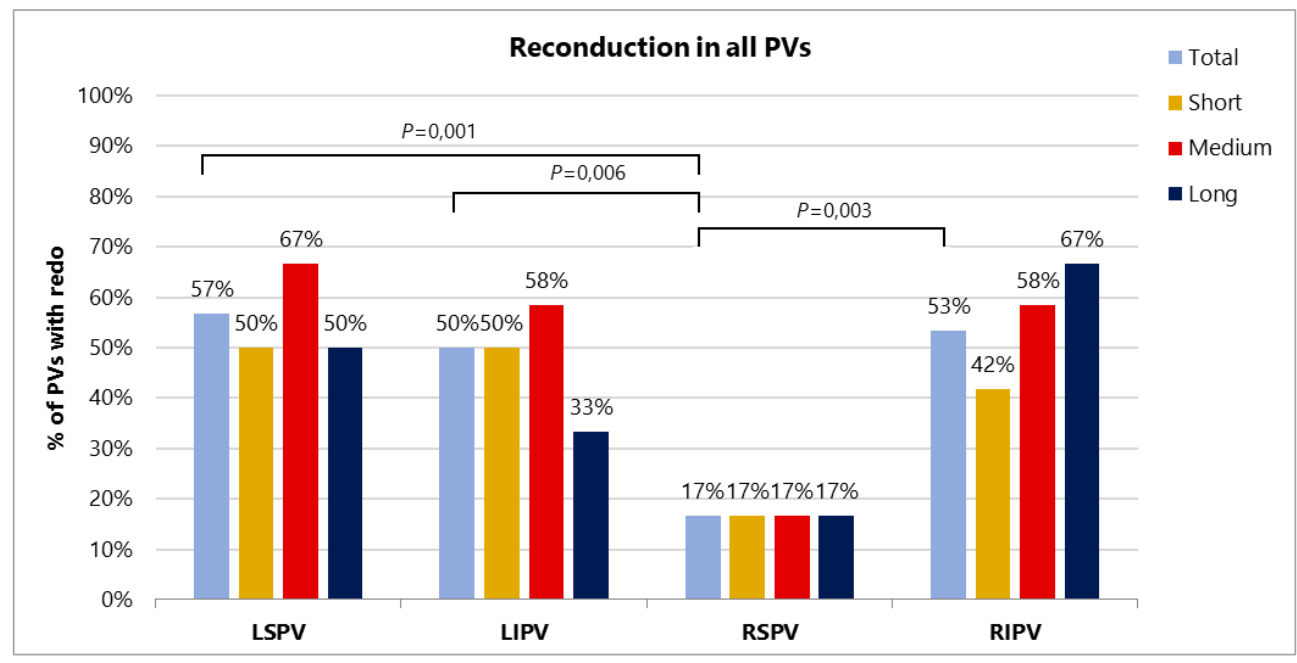

Figure 2 Recurrence distribution per PV for all patients Overall (= total) Per randomization group (short, medium, long) PV: Pulmonary Vein LS: Left Superior LI: Left Inferior RS: Right Superior Rl: Right Inferior

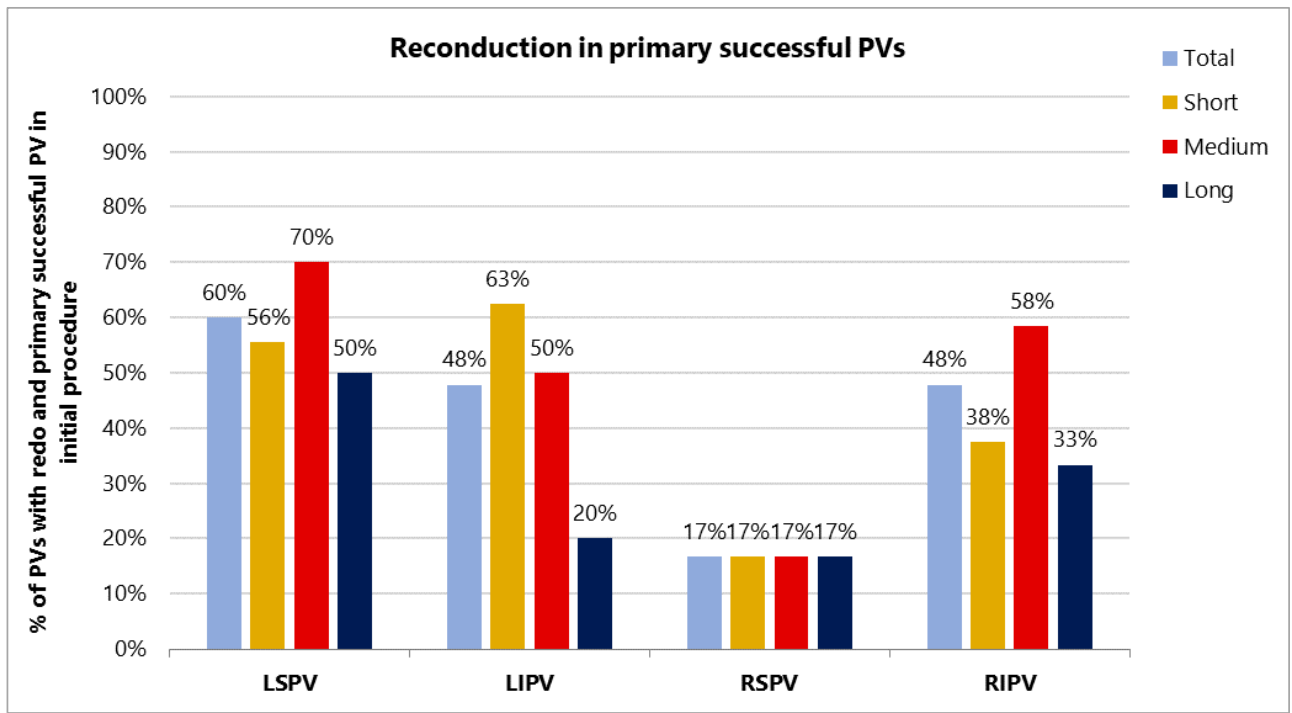

Figure 3 Recurrence distribution per PV for primary successful PV group Overall (total, group of primarily successful isolated PV's in initial procedure) Per randomization group (short, medium, long) PV: Pulmonary Vein LS: Left Superior LI: Left Inferior RS: Right Superior RI: Right 


\section{Application duration and freedom from AF}

This follow-up analysis shows a 1-year freedom from AF success rate of about $80 \%$, which is in accordance with success rates reported earlier ${ }^{3,13,14}$. The success rates for 1-year freedom from AF did not differ significantly between the randomization groups indicating that 1-year efficacy is not impaired by shortening application duration. However, a trend towards higher 1-year freedom from AF for the "long" group was found in the entire patient group. Similar results were seen in the CIRCADOSE study, the only other study performed prospectively in a randomized fashion comparing the standard to new dosing protocols. In that study, 2 min vs $4 \mathrm{~min}$ application duration showed a $73 \%$ vs $78 \%$ efficacy after 12 months $^{15}$.

\section{Reconduction per randomization group}

The per-PV-analysis indicated that shortening the cryoablation duration does not affect the reconduction rate in the individual PVs. However, a trend towards more durable lesions was noted in the "long" group for the LSPV, LIPV and RIPV (Figure 3). Recently Chen et al. published comparable results where a freeze duration of 240 versus $180 \mathrm{~s}$ was associated with significantly increased lesion durability, particularly at the left-sided PVs ${ }^{16}$. Here the low number of PVs per group in our study should be considered. Assessment of larger redo-cohorts is needed to confirm these results.

\section{Reconduction per PV}

Isolation assessment in redo-procedures showed that the LSPV had the most and RSPV the least reconductions, significantly less when compared to all three other PVs. The reconduction per PV after second-generation cryoballoon PVI showed mixed results in earlier studies. One study demonstrated that the RSPV is most prone to reconduction, in other studies the RIPV showed most reconductions, attributed to the challenging anatomy of the RIPV in terms of occlusion ${ }^{17-19}$. A recent study by Martins et al. reported reconduction rates around 20\% in the left PVs and around $30 \%$ in the right $\mathrm{PVS}^{20}$. With patient numbers in the mentioned studies ranging from around 18 to 66 , our study with 30 patients is comparable. The different dosing protocols adopted in these studies may have accounted for differences in outcome.

\section{Phrenic nerve injury}

The first results of the 123-study showed an important reduction in PNI incidence from $6.8 \%$ and $6.5 \%$ in the long and medium group to $1.7 \%$ in the short group ${ }^{11}$. Although the number of PS patients differed between the randomization groups (Figure 1b) the RSPV showed an acute success rate of $92 \%$ which was similar to 
acute success rates for medium and long randomization groups. Hence, application duration reduction towards short applications (105 [101-108] s) for the RSPV resulted in less PNI and did not impair acute isolation"1. The current results show that 1-year success with shorter cryoapplications for the RSPV is not impaired either, justifying a shortened application duration in the RSPV to prevent from PNI. This adds to the growing awareness that all four PVs should be assessed separately regarding PV isolation and to find the parameters to predict lesion durability ${ }^{21}$.

\section{Conclusion}

Shortening cryoballoon application duration of the RSPV to less than 2 minutes results in less PNI while acute success (PVI) as well as 1-year success (freedom from AF) was not compromised. Therefore cryoballoon application duration for the RSPV could be shortened to prevent PNI. 


\section{References}

1. Calkins H, Hindricks G, Cappato R, et al. 2017 HRS/EHRA/ECAS/APHRS/SOLAECE expert consensus statement on catheter and surgical ablation of atrial fibrillation. Heart Rhythm. 2017;14(10):e275e444. doi:10.1016/j.hrthm.2017.05.012

2. Kirchhof P, Benussi S, Kotecha D, et al. 2016 ESC Guidelines for the management of atrial fibrillation developed in collaboration with EACTS. Eur Heart J. 2016;50(5):e1-e88.

doi:10.1093/ejcts/ezw313

3. Kuck K-HH, Brugada J, Fürnkranz A, et al. Cryoballoon or Radiofrequency Ablation for Paroxysmal Atrial Fibrillation. N Engl J Med. 2016;374(23):2235-2245. doi:10.1056/NEJMoa1602014

4. Tomaiko E, Tseng A, Su WW. Radiofrequency versus cryoballoon ablation for atrial fibrillation. Curr Opin Cardiol. 2020;35(1):13-19. doi:10.1097/HCO.0000000000000700

5. Aytemir K, Gurses KM, Yalcin MU, et al. Safety and efficacy outcomes in patients undergoing pulmonary vein isolation with second-generation cryoballoon. EP Europace. 2014;17(3):379-387. doi:10.1093/europace/euu273

6. Fürnkranz A, Bordignon S, Schmidt B, et al. Incidence and characteristics of phrenic nerve palsy following pulmonary vein isolation with the second-generation as compared with the firstgeneration cryoballoon in 360 consecutive patients. EP Europace. 2015;17(4):574-578. doi:10.1093/europace/euu320

7. Martins RP, Hamon D, Césari O, et al. Safety and efficacy of a second-generation cryoballoon in the ablation of paroxysmal atrial fibrillation. Heart Rhythm. 2014;11(3):386-393. doi:10.1016/j.hrthm.2014.01.002

8. Chierchia G-B, Mugnai G, Hunuk B, et al. Impact on clinical outcome of premature interruption of cryoenergy delivery due to phrenic nerve palsy during second generation cryoballoon ablation for paroxysmal atrial fibrillation. J Cardiovasc Electrophysiol. 2015;26(9):950-955. doi:10.1111/jce.12718

9. Osório TG, Coutiño HE, Brugada P, et al. Recent advances in cryoballoon ablation for atrial fibrillation. Expert Rev Med Devices. 2019;16(9):799-808. doi:10.1080/17434440.2019.1653181

10. Chun JKR, Bordignon S, Chen S, et al. Current Status of Atrial Fibrillation Ablation with Balloon Strategy. Korean Circ J. 2019;49(11):991. doi:10.4070/kcj.2019.0226

11. Molenaar MMD, Timmermans CC, Hesselink T, et al. Shorter cryoballoon applications times do effect efficacy but result in less phrenic nerve injury: Results of the randomized 123 study. Pacing Clin Electrophysiol. 2019;42(5):508-514. doi:10.1111/pace.13626

12. Ghosh J, Sepahpour A, Chan KH, et al. Immediate balloon deflation for prevention of persistent phrenic nerve palsy during pulmonary vein isolation by balloon cryoablation. Heart Rhythm. 2013;10(5):646-652. doi:10.1016/j.hrthm.2013.01.011

13. He X, Chen Y, Zhou Y, et al. One-Year Clinical Outcome of Pulmonary Vein Isolation Using the Second-Generation Cryoballoon: A Meta-Analysis. Pacing Clin Electrophysiol. 2016;39(2):182-189. doi:10.1111/pace.12787

14. Ciconte G, Coulombe N, Brugada P, et al. Towards a tailored cryo-pulmonary vein isolation. Lessons learned from second-generation cryoballoon ablation. Trends Cardiovasc Med. 2019;29(7):420-425. doi:10.1016/j.tcm.2018.11.009

15. Andrade JG, Champagne J, Dubuc M, et al. Cryoballoon or Radiofrequency Ablation for Atrial Fibrillation Assessed by Continuous Monitoring: A Randomized Clinical Trial. Circulation. 2019:1779-1788. doi:10.1161/circulationaha.119.042622

16. Chen S, Schmidt B, Bordignon S, et al. Impact of Cryoballoon Freeze Duration on Long-Term 
Durability of Pulmonary Vein Isolation: ICE Re-Map Study. JACC Clin Electrophysiol. 2019;5(5):551559. doi:10.1016/j.jacep.2019.03.012

17. Koektuerk B, Yorgun H, Koektuerk O, et al. Characterization of Electrical Reconnection Following Pulmonary Vein Isolation Using First- and Second-Generation Cryoballoon. PACE - Pacing Clin Electrophysiol. 2016;39(5):434-442. doi:10.1111/pace.12834

18. Heeger CH, Wissner E, Mathew S, et al. Once Isolated, Always Isolated? Incidence and Characteristics of Pulmonary Vein Reconduction after Second-Generation Cryoballoon-Based Pulmonary Vein Isolation. Circ Arrhythmia Electrophysiol. 2015;8(5):1088-1094. doi:10.1161/CIRCEP.115.003007

19. Bordignon S, Fürnkranz A, Perrotta L, et al. High rate of durable pulmonary vein isolation after second-generation cryoballoon ablation: Analysis of repeat procedures. EP Europace. 2015;17(5):725-731. doi:10.1093/europace/euu331

20. Martins RP, Galand V, Cesari O, et al. The second generation cryoballoon has improved durable isolation of left but not right pulmonary veins: New insights from a multicentre study. EP Europace. 2018;20(7):1115-1121. doi:10.1093/europace/eux200

21. Miyazaki S, Kajiyama T, Watanabe T, et al. Predictors of durable pulmonary vein isolation after second-generation cryoballoon ablation with a single short freeze strategy - Different criteria for the best freeze of the 4 individual PVs. Int J Cardiol. 2020;301:96-102.

doi:10.1016/j.ijcard.2019.11.089 
CHAPTER 5 5

CHAPTER 5

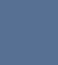

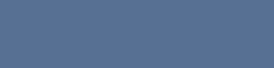

(

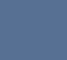

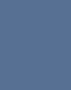

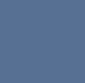

(1)

P

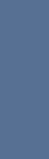

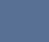

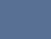

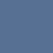

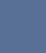

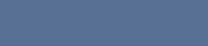

(

stis 


\section{HIGH INCIDENCE OF (ULTRA)LOW \\ OESOPHAGEAL TEMPERATURES DURING \\ CRYOBALLOON PULMONARY VEIN \\ ISOLATION FOR ATRIAL FIBRILLATION}

Marleen MD Molenaar, Tim Hesselink, Marcoen F Scholten, Karin Kraaier, Donald E Bouman, Marjolein Brusse-Keizer, Y Jeroen Stevenhagen, Pascal HFM van Dessel, Bernard ten Haken, Jan G Grandjean, Jurren M van Opstal

Accepted (with revisions) for publication in Netherlands Heart Journal 


\section{Abstract}

Background Low oesophageal temperatures (OTs) during cryoballoon pulmonary vein (PV) isolation (PVI) have been associated with complications. This study assessed the incidence of low OT in clinical practice during cryoballoon PVI and verified possible predictive values for low OT.

Methods Consecutive patients who underwent PVI using the second generation cryoballoon were retrospectively included. Distance from the oesophagus to the different PVs (OP distance), Body Mass Index (BMI), sex, age, balloon temperature and application time were studied as potential predictor for low OTs. A preprocedural CT was performed to determine OP distance. OT was measured using an oesophageal temperature probe. Applications were prematurely ended if OT reached $<16^{\circ} \mathrm{C}$. Low and ultralow OT were defined as OT $<20$ and $<16^{\circ} \mathrm{C}$.

Results Two hundred and four patients were included. Low OT was observed in 54 patients (26\%) and 27 patients (13\%) reached ultralow OTs. OP distance was the only predictor for low OTs after multivariate analysis. A cut-off value of $19 \mathrm{~mm}$ showed $96.2 \%$ sensitivity and $37.8 \%$ specificity in predicting low OTs. No clinically relevant relation was found between low OTs and BMI, age, sex, balloon temperature or application duration.

Conclusions The incidence of low OT was $26 \%$ for cryoballoon PVI. OP distance was the only predictor for low OTs. Since OP distance $<19 \mathrm{~mm}$ for any PV was present in all patients we recommend routine OT measurement during PVI cryoballoon therapy to prevent oesophageal related complications. 


\section{Background}

Pulmonary vein (PV) isolation (PVI) is an established treatment option for atrial fibrillation (AF). Cryoballoon catheters are increasingly used to perform PVI as a successful alternative to point-by-point radiofrequency ablation.

However, cyrothermal energy can reach the surrounding tissues, potentially causing collateral damage. Oesophageal lesions, atrio-oesophageal fistulae and vagal nerve injury, resulting in gastroparesis, have been related to low oesophageal temperatures (OT's) $)^{1-7}$. OT's $\leq 12^{\circ} \mathrm{C}$ have shown a $100 \%$ sensitivity and $92 \%$ specificity in prediction of gastro-oesophageal lesions formation ${ }^{3}$. Interruption of cryoablation at an OT of $15^{\circ} \mathrm{C}$ has been associated with reduced incidence of oesophageal injury ${ }^{8}$.

In clinical practice low OTs emerge on a regular base. Finding predictive parameters for low OT's would enable taking precautions to prevent collateral damage. This study aimed to retrospectively assess the incidence of "low" and "ultralow" OT, defined as OT $\angle 20^{\circ} \mathrm{C}$ respectively $<16^{\circ} \mathrm{C}$, in clinical practice during regular PVI using the second generation cryoballoon. Furthermore we hypothesized that predictive parameters for (ultra)low temperatures could be found in the anatomical position of the oesophagus in relation to the different PVs, BMI, age, sex, balloon temperature and/or application time.

\section{Methods}

\section{Patients}

Patients accepted for cryoballoon PVI according to current international guidelines at the Medisch Spectrum Twente (Enschede, the Netherlands) were included in this single centre retrospective study ${ }^{9}$. Need for informed consent was waived by the medical ethical committee and this study complies with the Declaration of Helsinki.

\section{Cardiac CT PVI protocol}

Pre PVI image acquisition by cardiac CT angiography was performed on a 64 detector Toshiba Aquilion CT-scanner (Toshiba Medical Systems, Tokyo, Japan). If possible, CT scanning was synchronized with the electrocardiogram. Parameters were field of view $500 \mathrm{~mm}, 120 \mathrm{kV}, 300 \mathrm{mAs}$ (automatically adjusted to the cardiac cycle typically at $75 \%$. Acquisition was performed using a $0.5 \mathrm{~mm}$ slice thickness and $0.3 \mathrm{~mm}$ slice interval.

A bolus of $80 \mathrm{~mL}$ iodinated contrast agent (Visipaque $320 \mathrm{mg} \mathrm{l} / \mathrm{mL}$ ) was injected at the antecubital vein at $3 \mathrm{~mL} / \mathrm{s}$ followed by saline chaser of $20 \mathrm{~mL}$. Bolus tracking was 


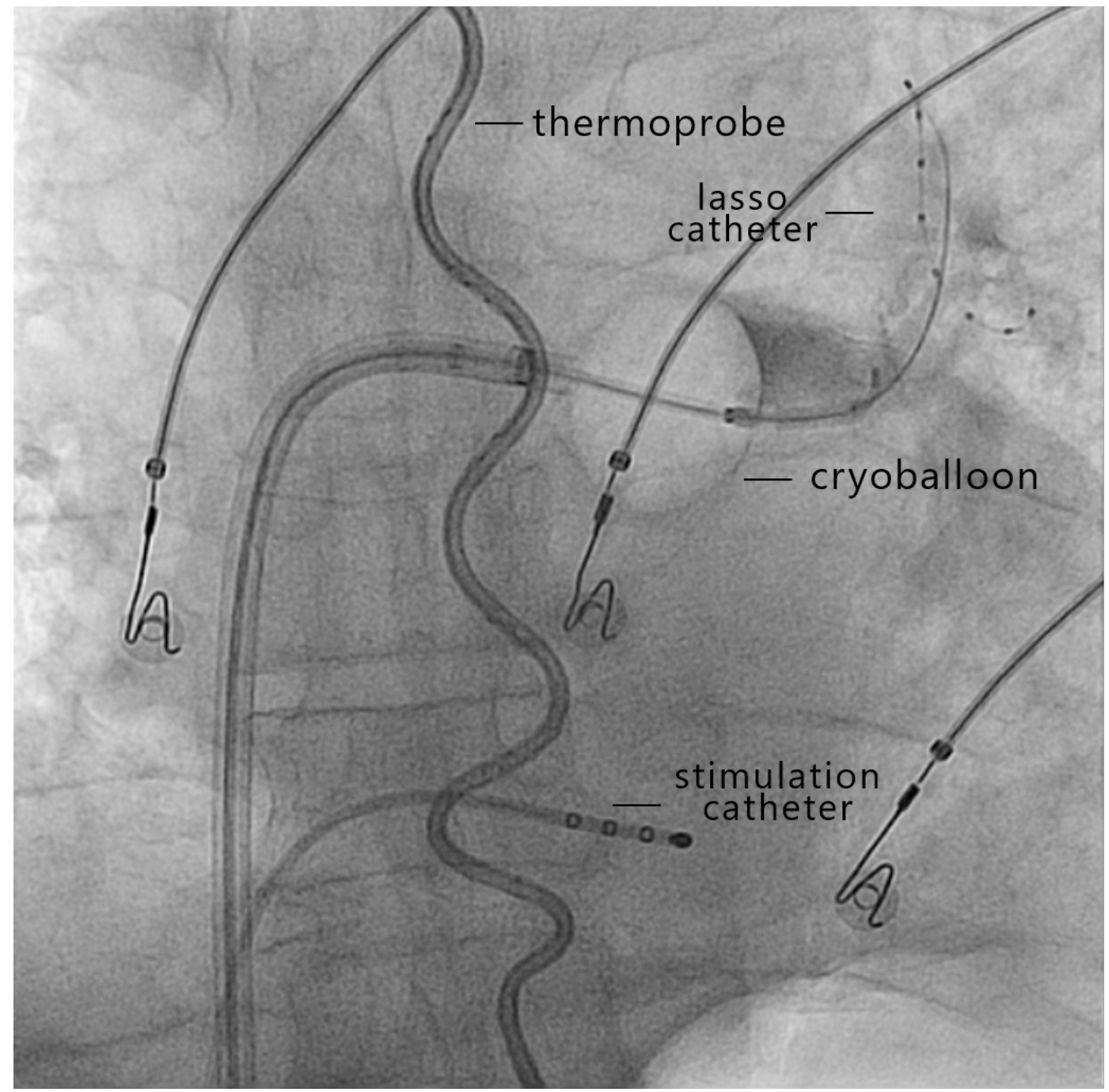

Figure 1 Position of the thermoprobe (s-shaped) in the oesophagus with cryoballoon positioned at the antrum of the left superior pulmonary vein with the lasso catheter inside this pulmonary vein and contrast dye being injected in the pulmonary vein (right upper quadrant). The stimulation catheter is positioned in the coronary sinus. 
a.

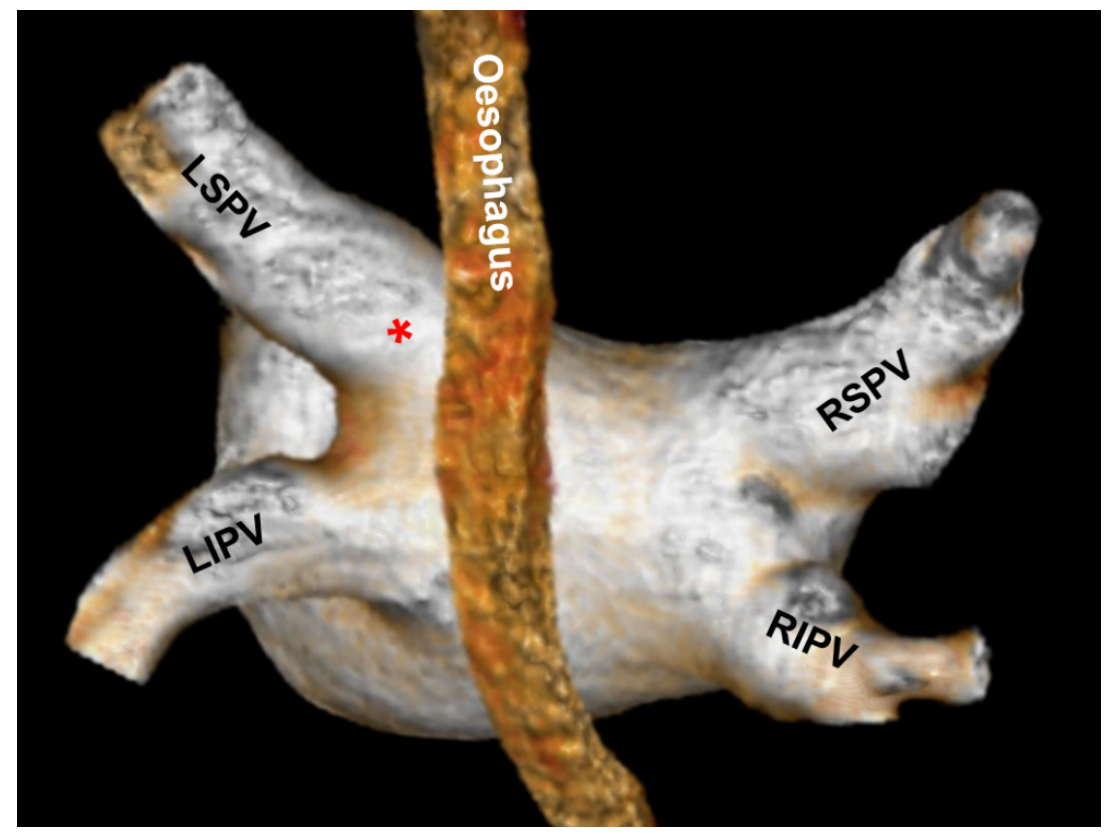

b.

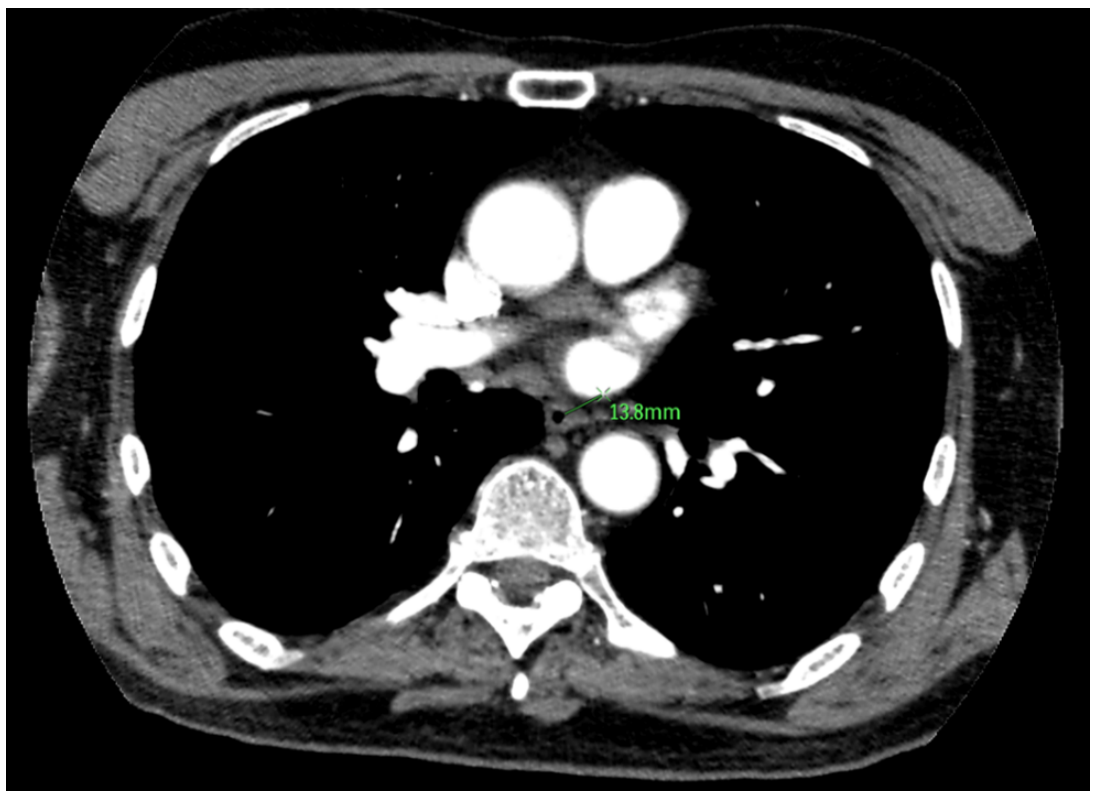

Figure 2 Shortest oesophagus to PV (OP) distance measurement. First we looked in the CT images for $a$. the origin of the os, identified as the indentation in the posterior wall, caused by the angulation of the pulmonary vein compared to the atrial wall. The angulation between the PV and the atrium can be well distinguished in this 3D view. The distance from identified angulation $\left(^{*}\right)$ to the oesophagus in the axial plane represents the closest OP distance. b. This distance was measured $(13.8 \mathrm{~mm})$. 
performed until a region of interest of $>170$ Hounsfield Units was measured in the left atrium.

\section{PVI procedure}

All procedures were performed under general anaesthesia with continuous arterial blood pressure monitoring. Heparin was administered to achieve an activated clotting time of $>300$ seconds during the procedure. A temperature probe was inserted into the oesophagus under fluoroscopic guidance. In the first 79 patients a temperature probe with 3 thermocouples separated by $10 \mathrm{~mm}$ (SensiTherm ${ }^{\mathrm{TM}}$, St Jude Medical Inc., MN, USA) was used, the position of this temperature probe was adjusted to the fluoroscopic position of the balloon during each application. In the last 126 patients a S-shaped temperature probe with 12 electrically insulated temperature sensors (CIRCA S-CATH ${ }^{\mathrm{Tm}}$, CIRCA Scientific, CO, USA) was used (Figure 1). The use of a 23 or $28 \mathrm{~mm}$ balloon (Arctic Front Advance ${ }^{\mathrm{TM}}$ ) was based on the PV diameters. Optimal positioning of the balloon was performed integrating the preperformed CT images in a 3D system (Philips, The Netherlands) or by angiography of the $P V s^{10}$.

During cryoablation of the right sided PVs the right phrenic nerve was continuously stimulated by pacing from the superior caval vein or the right subclavian vein. If diminished diaphragm excursion was observed during cryotherapy, the application was stopped immediately using the double stop technique ${ }^{11}$. If reaching $0 \mathrm{Ts}<16^{\circ} \mathrm{C}$, the application was stopped prematurely ${ }^{8}$. A subsequent application was not applied until OT reached $>30^{\circ} \mathrm{C}$ again.

Initially two applications were performed and target application time did not exceed 240 s in all but 5 applications. Successful isolation of a PV was proven by entrance and exit block. If isolation was unsuccessful after the initial two applications, ablation was continued until complete isolation was achieved. At the end of the procedure, possible dormant conduction was evaluated using adenosine.

\section{CT Measurements}

The CT we used for was primarily performed to assess the PV anatomy prior to PVI. Based on the anatomy a patient was scheduled for Cryoballoon or RF. For this study these pre-PVI acquired CT images were used to measure the shortest distance between the oesophagus and the os of every PV (OP distance). To measure this OP distance, the origin of the os was identified as the indentation in the posterior wall, caused by the angulation of the PV compared to the atrial wall (Figure 2a). since the oesophagus runs perpendicular to the axial plane, the distance from the 
identified angulation to the oesophagus in the axial plane represents the closest OP distance. This distance was measured (Figure 2b).

\section{Statistical analysis}

Low OT was defined as $\mathrm{OT}<20^{\circ} \mathrm{C}$ and ultralow OT as $\mathrm{OT}<16^{\circ} \mathrm{C}$. Continuous variables are reported as mean with SD (normally distributed) or as median with interquartile range (non-parametric data). Categorical variables are displayed as numbers. Comparison of baseline characteristics between patients with and without low OT was performed using an independent T-test or Mann-Whitney $U$ test as appropriate for continuous variables. For comparison of categorical variables the Chi-square or Fisher exact test was used.

Correlation coefficients were calculated (Pearson or Spearman correlation tests) to test the association of the potential predictors, OP distance, BMI, age, sex, balloon temperature and application time, with the continuous OT. A correlation coefficient $<0.25$ was considered clinically not relevant. Univariate logistic regression analyses were performed to select predictors for low OT. All variables associated with $p<0.10$ were entered in a multivariate logistic regression analysis. Subsequently variables with the highest $p$-values were deleted until the fit of the model decreased significantly (based on -2loglikelihood). A 2-sided $P$ value of $<0.05$ was considered statistically significant. Statistical analyses were performed with SPSS v.22.

\section{Results}

\section{Incidence}

Two hundred and four patients were included. Baseline characteristics of the study cohort are shown in Table 1. Median time between CT acquisition and PVI was 66 days (IQR 38-99). Figure 3 shows the distribution of OTs reached. In 54 patients (26\%), two with the $23 \mathrm{~mm}$ balloon, OTs below $20^{\circ} \mathrm{C}$ were reached. In 27 patients (13\%) the lowest OT was $<16^{\circ} \mathrm{C}$ and in one patient OT dropped below $10^{\circ} \mathrm{C}\left(7.6^{\circ} \mathrm{C}\right)$. In 23 patients applications were prematurely stopped because of (ultra)low OT. In 4 patients with low OT's, applications were not prematurely terminated. Low OT's were reached after regular application time was completed, due to the latency effect. There were no significant differences in baseline characteristics between the (ultra) low OT group and the group without low OT. In all but five patients the $28 \mathrm{~mm}$ balloon was used, in six patients both the $28 \mathrm{~mm}$ and the $23 \mathrm{~mm}$ balloon were used. Low OT's were found $8 \%$ of the PVs in which the SensiTherm was used and in $9 \%$ of the PVs in which the Circa S probe was used $(\mathrm{p}=0.67)$. In 168 patients a CT was available for measurement of OP distance. Lowest OTs occurred in the left inferior 
Table 1 Baseline characteristics ( $n=204)$

\begin{tabular}{rll}
\hline Parameter & $\begin{array}{l}\text { No low OT } \\
(\mathrm{n}=148)\end{array}$ & $\begin{array}{l}\text { Low OT } \\
(\mathrm{n}=56)\end{array}$ \\
\hline Age (years) & $56 \pm 11$ & $58 \pm 12$ \\
\hline Gender: male $\mathrm{n}(\%)$ & $105(71)$ & $36(64)$ \\
\hline LA diameter $\mathrm{n}(\%)^{\dagger}$ & & \\
\hline Normal & $126(85)$ & $46(82)$ \\
\hline Mildly dilated & $17(12)$ & $7(13)$ \\
\hline Moderately dilated & $3(2)$ & $0(0)$ \\
\hline Severely dilated & $1(1)$ & $1(2)$ \\
\hline Unknown & $1(1)$ & $2(4)$ \\
\hline Medical history n (\%) & & \\
\hline Hypertension & $45(30)$ & $12(21)$ \\
\hline Diabetes mellitus & $5(3)$ & $1(2)$ \\
\hline Coronary Artery Disease & $12(8)$ & $4(7)$ \\
\hline Stroke & $2(1)$ & $0(0)$ \\
\hline LA: Left Atrium, OT: Oesophagus Temperature
\end{tabular}

LA: Left Atrium, OT: Oesophagus Temperature

${ }^{+}$LA diameter ranges for $\mathrm{cc} / \mathrm{m}^{2}$ as in Lang et al. $2015^{32}$ Normal $16-34 \mathrm{cc} / \mathrm{m}^{2}$ Mildly dilated $35-41 \mathrm{cc} / \mathrm{m}^{2}$ Moderately dilated $32-48 \mathrm{cc} / \mathrm{m}^{2}$ Severely dilated $>48$ $\mathrm{cc} / \mathrm{m}^{2}$
Table 2 Number of lowest OT per patient sorted by PV of occurrence

\begin{tabular}{llll}
\hline PV & \multicolumn{3}{c}{ Temperature $\left({ }^{\circ} \mathrm{C}\right)$} \\
\hline & $20-16$ & $16-10$ & $10-0$ \\
\cline { 2 - 4 } LSPV & 0 & 1 & 0 \\
\hline LIPV & 18 & 16 & 1 \\
\hline RSPV & 2 & 0 & 0 \\
\hline RIPV & 7 & 9 & 0 \\
\hline
\end{tabular}

OT; Oesophagus Temperature PV; Pulmonary vein LS; Left Superior LI; Left Inferior RS; Right Superior RI; Right Inferior

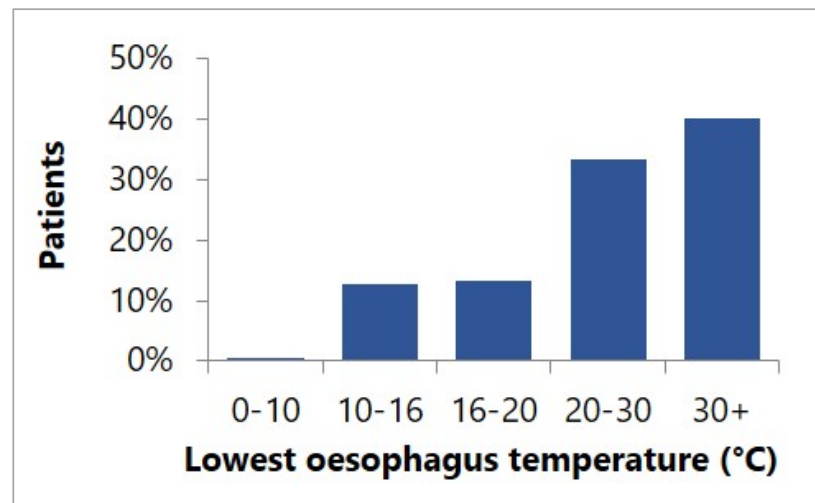

Figure 3 Occurrence (in \% of patients) of lowest oesophagus temperatures (in ${ }^{\circ} \mathrm{C}$ ) during $\mathrm{PVI}$ 
(LI) and right inferior (RI) PVs (Table 2). Median OP distance was 8.7[5.4-12.3] mm for left superior (LS) PV, 7.6[4.7-11.8] $\mathrm{mm}$ for the LIPV, 29.3[23.7-34.7] $\mathrm{mm}$ for the right superior (RS) PV and 17.0[11.8-22.7] $\mathrm{mm}$ for the RIPV. During regular clinical followup for 1 year no clinical consequences were demonstrated within the group of (ultra)low OT.

\section{Predictors}

No clinically relevant correlation with OT was found for BMI, age, sex, balloon temperature or application time. A significant and clinically relevant correlation of 0.405 was found for OP distance and OT (Figure 4). Univariate logistic regression analysis showed that low OTs ( $<16 \%)$ were negatively associated with OP distance (OR 0.85; $\mathrm{Cl} 0.80-0.90 ; \mathrm{p}<0.001$ ), and positively associated with balloon temperature (OR 1.08; Cl 1.03-1.12; $p=0.001)$. BMI (OR 0.92; Cl 0.85-1.01; $p=0.072$ ), age (OR 1.01; Cl 0.991.04; $p=0.41$ ), male gender (OR $1.56 \mathrm{Cl} 0.87-2.77 \mathrm{p}=0.14$ ), and application time (OR 0.99; $\mathrm{Cl}$ 0.99-1.00; $\mathrm{p}=0.075)$ were not independently associated. OP distance, balloon temperature, $\mathrm{BMI}$ and application time were included in the multivariate analysis based on the level of significance.

In multivariate logistic regression analysis, OP distance (OR $0.8495 \% \mathrm{Cl} 0.791-0.895$ $\mathrm{p}<0.000)$ and application time (OR $0.9995 \% \mathrm{Cl} 0.985-0.999 \mathrm{p}=0.020)$ were the only independent predictors of low OT's. Since the application time was shortened in the group of patients reaching ultralow OT's, a sensitivity analysis was performed and ultralow OT's were excluded. The sensitivity analysis showed that there was no predicting effect of application time. Therefore, the OP distance was the single independent predictor for low OT's. Assessment of the sensitivity and specificity was performed for several cut-off values (Table 3). Since this parameter would be used for prevention the focus should be on a high sensitivity, preferably $>95 \%$. Therefore we selected a cut-off value of $19 \mathrm{~mm}$ for OP distance. This showed a $96.2 \%$ sensitivity and $37.8 \%$ specificity in predicting low OT's (Table $3 \& 4$ ). An OP distance $<19 \mathrm{~mm}$ was present in all patients in at least one PV.

\section{Discussion}

In our study population second generation cryoballoon PVI shows low OTs, below $20^{\circ} \mathrm{C}$, in 54 patients $(26 \%)$. In half of them ultralow OTs, below $16^{\circ} \mathrm{C}$, were measured despite the cessation of cryotherapy at $16^{\circ} \mathrm{C}$. Multivariate analysis identified OP distance as the only predictor for low OTs. A cut-off value of $19 \mathrm{~mm}$ showed a $96.2 \%$ sensitivity and a $37.8 \%$ specificity for predicting low OTs. No clinically relevant 
Table 3 Sensitivity and specificity for OP distances cut-off values

\begin{tabular}{lll}
\hline $\begin{array}{l}\text { OP distance } \\
\text { cut-off }(\mathrm{mm})\end{array}$ & $\begin{array}{l}\text { Sensitivity } \\
(\%)\end{array}$ & $\begin{array}{l}\text { Specificity } \\
(\%)\end{array}$ \\
\hline 15 & 86.5 & 47.8 \\
\hline 18 & 92.3 & 40.3 \\
\hline 19 & 96.2 & 37.8 \\
\hline 20 & 96.2 & 35.2 \\
\hline 23 & 98.1 & 28.8 \\
\hline
\end{tabular}

OP; Oesophagus to Pulmonary vein
Table 4 Occurrence of low OT per PV for OP distances $<$ and $>19 \mathrm{~mm}$

\begin{tabular}{lll}
\hline OP distance & LOW OT & No Low OT \\
\hline$<19 \mathrm{~mm}$ & 50 & 361 \\
\hline$>19 \mathrm{~mm}$ & 2 & 219 \\
\hline OT; Oesophagus Temperature OP; \\
Oesophagus to Pulmonary vein; PV \\
Pulmonary Vein
\end{tabular}
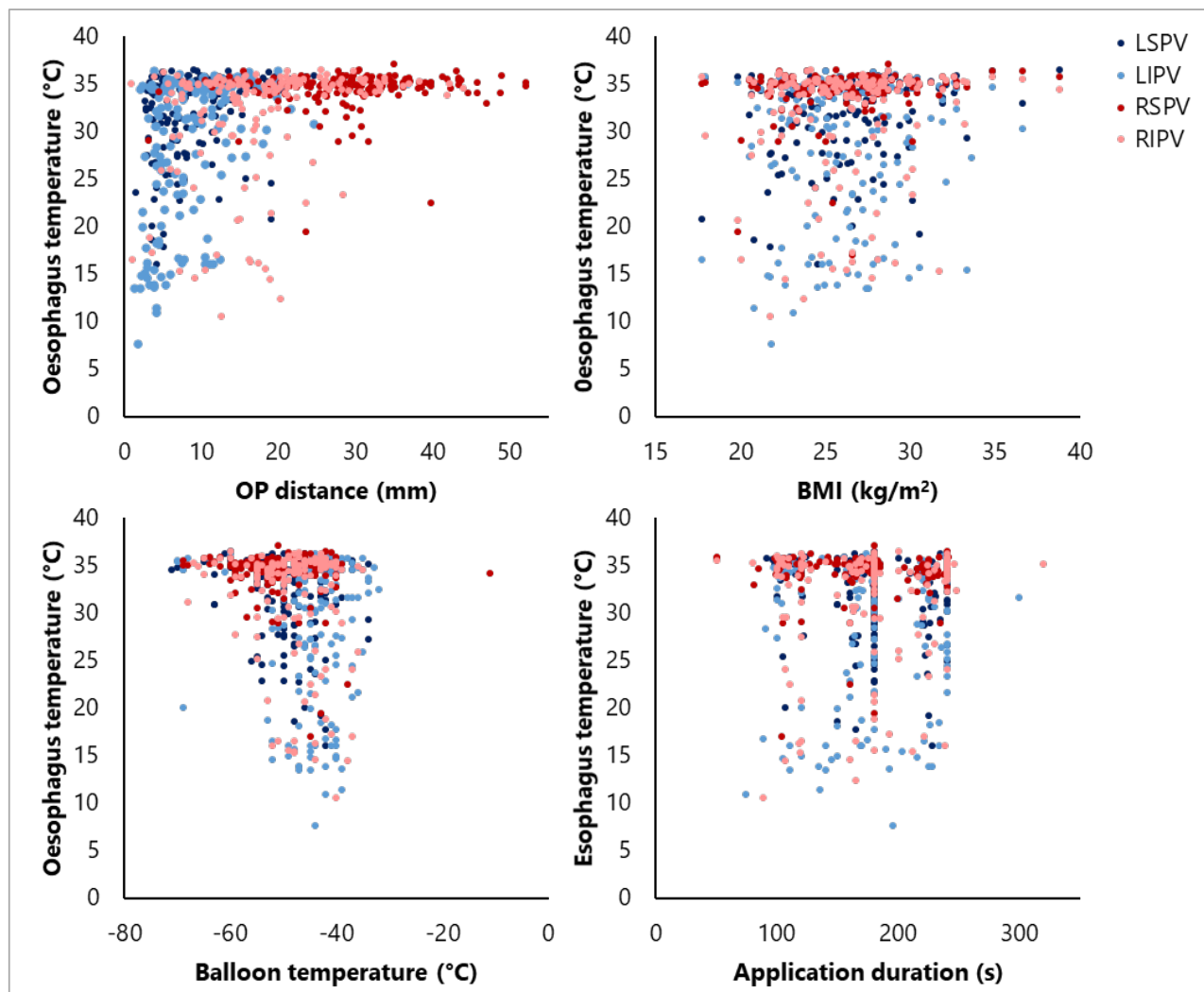

Figure 4 Relation of lowest oesophagus temperature with corresponding distance from PV to oesophagus (OP distance), BMI, balloon temperature and application duration. 
relation was found between low OTs and BMI, age, sex, balloon temperature or application duration.

With the introduction of the second generation balloon the PVI success had increased ${ }^{12,13}$. However the potential of inadvertent collateral injury has increased too since cryothermal energy disperses into the surrounding tissues. This involves damage to the oesophagus (oesophageal lesions and even fistulas), as well as damage to the vagal and phrenic nerves but also bronchial effects have been described ${ }^{1-7,14,15}$.

Several studies performing systematic postprocedural esophagoscopy post PVI

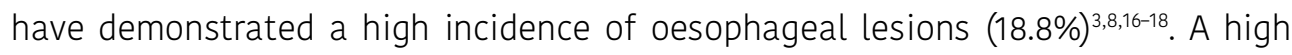
incidence of gastric motility disturbance, which can result in gastroparesis, has been described too $(17.3 \%)^{6,7,19,20}$. This relates to the vagal nerve which is situated in close proximity to the heart, spreading out on the oesophagus in a web-like structure, innervating the stomach, providing gastric motility, and the pyloric sphincter.

In our study the OP distance, as determined on pre-PVI performed CT images, was the single predictor of low OT's, with a cut-off value of $20.0 \mathrm{~mm}$ showing a high sensitivity. However, in every patient at least one of the PV's showed an OP distance $<19 \mathrm{~mm}$ which implies that a temperature probe should be routinely used. Unfortunately, the specificity of this predictor was fairly low. An explanation could be the variability of the oesophageal position in time. Research in that field, with small numbers, showed evidence for a fixed as well as a variable position ${ }^{21-23}$.

The relation between OP distance and OT has only been shown for radiofrequency PVI and ultrasound balloon PVI so far, but seems trivial24,25. Martinek already showed oesophagus to left atrium distance as the single predictor for oesophageal ulcerations in radiofrequency $\mathrm{PVI}^{26}$. Recently, Miyazaki assessed the relation between OP distance and gastric hypomotility in cryoballoon PVI and found a cutoff value of $18.2 \mathrm{~mm}$ for the RIPV ( $88.1 \%$ sensitivity $77.8 \%$ specificity) $)^{19}$. It was also suggested that the anatomical relation between PV's and aorta may identify patients prone for low OT's ${ }^{19,27}$.

Another explanation could be that the pressure applied to the PV, when occluding it using the balloon, changes the relationship between the LA and the oesophagus. In our study, low and ultralow OT occurred predominantly in the inferior PVs (Table 2) which can be explained by the anatomic course of the PVs. As the inferior PVs run posteriorly, the balloon is being pushed towards the oesophagus in these PVs. 
OT guidance of cryoballoon PVI has shown a significant reduction of thermal oesophageal lesions and interrupting applications at an OT of $15^{\circ} \mathrm{C}$ has been suggested to prevent from oesophageal injury ${ }^{8}$. Our study shows that low OT is found in a considerable number of patients during cryoballoon PVI, but the use of temperature probes is still not routinely used nor recommended.

In the first 79 patients a straight temperature probe with 3 thermocouples (SensiTherm) was used. A concern of this probe is the possible underestimation of the ET. It could be positioned contralateral to the side where the energy is applied or could be floating free in the oesophagus ${ }^{28,29}$. Furthermore the point of coldest temperature could be in between the thermocouples. During the study period the most recent thermoprobe (Circa S-Cath) was introduced and some of these disadvantages seem to be eliminated ${ }^{30}$ (Figure 1). It contains 12 electrically insulated temperature sensors which are positioned with smaller spacing. Due to the S-shape of the probe it touches the oesophagus walls and covers the whole length of the oesophagus in relation to the PVs which prevents the need to reposition the probe during the procedure. Therefore we switched to the Circa SCath in the remaining 125 patients. The usage of two different probes might have had an effect on the registered OT's. However no differences between these two groups were found for occurrence of low OT.

Balloon temperatures are often used as a surrogate metric for OTs and for possible low temperatures in adjacent structures. The absence of clinically significant correlation between minimum balloon temperature and lowest OT found in our study is in accordance with earlier reports on this subject ${ }^{3,8}$.

Despite the cessation of cryotherapy when OT reached $<16^{\circ} \mathrm{C}$, OT's far below $16^{\circ} \mathrm{C}$ were seen. This decline in OT even after the freeze cycle was terminated has been reported upon by Deiss et al. ${ }^{31}$. It is also known as "the latency effect". The maximum latency effect found in that study was a decline of $6.4^{\circ} \mathrm{C}$. In our study an even larger maximum latency effect of $8.4^{\circ} \mathrm{C}\left(16\right.$ to $\left.7.6^{\circ} \mathrm{C}\right)$ was seen. It underlines the importance of this effect when applying safety cut-offs.

\section{Study Limitations}

The existence of variability in the oesophageal position is still in debate, research with small numbers showed evidence for a fixed as well as a variable position 21-23,30. If the oesophageal position indeed has active dynamics, this could have affected our results. If serious migration of the oesophagus between the moment the CT 
was acquired and the ablation was performed is possible, this could have affected the OP distance and therefore the predictive value of it.

No endoscopic inspection was performed to assess the possible extent of oesophageal injury. This has been addressed in prior studies which uniformly demonstrated a relation between low OT and oesophageal lesions ${ }^{3,8,16}$.

The usage of two different probes might have had an effect on the registered OT's. However no differences between these two groups were found for occurrence of low OT.

Finally, the retrospective design has to be taken into account.

\section{Conclusion}

Second generation cryoballoon PVI results in $\mathrm{OT}<20^{\circ} \mathrm{C}$ in $26 \%$ of patients. Half of them even showed $\mathrm{OT}<16^{\circ} \mathrm{C}$. Multivariate analysis identified OP distance as the only predictor for low OTs. No clinically relevant relation was found between low OTs and BMI, age, sex, balloon temperature or application duration. A cut-off value for OP distance of $19 \mathrm{~mm}$ showed a $96.2 \%$ sensitivity and a $37.8 \%$ specificity for predicting low OTs. As OP distance $<19 \mathrm{~mm}$ for any PV was present in all patients we recommend routine OT measurement in all patients during cryoballoon therapy to prevent oesophageal related complications. 


\section{References}

1. Casado-Arroyo R, Chierchia GB, Conte G, et al. Phrenic nerve paralysis during cryoballoon ablation for atrial fibrillation: A comparison between the first- and second-generation balloon. Heart Rhythm. 2013;10(9):1318-1324. doi:10.1016/j.hrthm.2013.07.005

2. Miyazaki S, Nakamura H, Taniguchi H, et al. Esophagus-Related Complications During SecondGeneration Cryoballoon Ablation-Insight from Simultaneous Esophageal Temperature Monitoring from 2 Esophageal Probes. J Cardiovasc Electrophysiol. 2016;27(9):1038-1044. doi:10.1111/jce.13015

3. Fürnkranz A, Bordignon S, Schmidt B, et al. Luminal esophageal temperature predicts esophageal lesions after second-generation cryoballoon pulmonary vein isolation. Heart Rhythm. 2013;10(6):789-793. doi:10.1016/j.hrthm.2013.02.021

4. John RM, Kapur S, Ellenbogen KA, et al. Atrioesophageal fistula formation with cryoballoon ablation is most commonly related to the left inferior pulmonary vein. Heart Rhythm. 2017;14(2):184-189. doi:10.1016/j.hrthm.2016.10.018

5. Kuwahara T, Takahashi A, Takahashi Y, et al. Clinical characteristics and management of periesophageal vagal nerve injury complicating left atrial ablation of atrial fibrillation: lessons from eleven cases. J Cardiovasc Electrophysiol. 2013;24(8):847-851. doi:10.1111/jce.12130

6. Guiot A, Savouré A, Godin B, et al. Collateral nervous damages after cryoballoon pulmonary vein isolation. J Cardiovasc Electrophysiol. 2012;23(4):346-351. doi:10.1111/j.1540-8167.2011.02219.x

7. Aksu T, Golcuk S, Guler TE, et al. Gastroparesis as a Complication of Atrial Fibrillation Ablation. Am J Cardiol. 2015;116(1):92-97. doi:10.1016/j.amjcard.2015.03.045

8. Fürnkranz A, Bordignon S, Böhmig M, et al. Reduced incidence of esophageal lesions by luminal esophageal temperature-guided second-generation cryoballoon ablation. Heart Rhythm. 2015;12(2):268-274. doi:10.1016/j.hrthm.2014.10.033

9. Camm AJ, Lip GGYH, De Caterina R, et al. 2012 focused update of the ESC Guidelines for the management of atrial fibrillation: an update of the 2010 ESC Guidelines for the management of atrial fibrillation. Developed with the special contribution of the European Heart Rhythm Association. Eur Heart J. 2012;33(10):2719-2747. doi:10.1093/eurheartj/ehs253

10. Stevenhagen J, Van Der Voort PH, Dekker LRC, et al. Three-dimensional CT overlay in comparison to CartoMerge for pulmonary vein antrum isolation. J Cardiovasc Electrophysiol. 2010;21(6):634639. doi:10.1111/j.1540-8167.2009.01665.x

11. Ghosh J, Sepahpour A, Chan KH, et al. Immediate balloon deflation for prevention of persistent phrenic nerve palsy during pulmonary vein isolation by balloon cryoablation. Heart Rhythm. 2013;10(5):646-652. doi:10.1016/j.hrthm.2013.01.011

12. Liu J, Kaufmann J, Kriatselis C, et al. Second generation of cryoballoons can improve efficiency of cryoablation for atrial fibrillation. Pacing Clin Electrophysiol. 2015;38(1):129-135. doi:10.1111/pace.12538

13. Aytemir K, Gurses KM, Yalcin MU, et al. Safety and efficacy outcomes in patients undergoing pulmonary vein isolation with second-generation cryoballoon. EP Europace. 2014;17(3):379-387. doi:10.1093/europace/euu273

14. Verma N, Gillespie CT, Argento AC, et al. Bronchial effects of cryoballoon ablation for atrial fibrillation. Heart Rhythm. 2017;14(1):12-16. doi:10.1016/j.hrthm.2016.10.012

15. van Opstal JM, Timmermans C, Blaauw Y, et al. Bronchial erosion and hemoptysis after pulmonary vein isolation by cryoballoon ablation. Heart Rhythm. 2011;8(9):1459. doi:10.1016/j.hrthm.2010.06.024 
16. Metzner A, Burchard A, Wohlmuth P, et al. Increased incidence of esophageal thermal lesions using the second-generation 28-mm cryoballoon. Circ Arrhythm Electrophysiol. 2013;6(4):769-775. doi:10.1161/CIRCEP.113.000228

17. Fürnkranz A, Chun KRJ, Metzner A, et al. Esophageal endoscopy results after pulmonary vein isolation using the single big cryoballoon technique. J Cardiovasc Electrophysiol. 2010;21(8):869874. doi:10.1111/j.1540-8167.2010.01739.x

18. Ahmed H, Neuzil P, D’Avila A, et al. The esophageal effects of cryoenergy during cryoablation for atrial fibrillation. Heart Rhythm. 2009;6(7):962-969. doi:10.1016/j.hrthm.2009.03.051

19. Miyazaki S, Nakamura H, Taniguchi H, et al. Gastric hypomotility after second-generation cryoballoon ablation-Unrecognized silent nerve injury after cryoballoon ablation. Heart Rhythm. 2017;14(5):670-677. doi:10.1016/j.hrthm.2017.01.028

20. Jung HK, Choung RS, Locke GR, et al. The Incidence, Prevalence, and Outcomes of Patients With Gastroparesis in Olmsted County, Minnesota, From 1996 to 2006. Gastroenterology. 2009;136(4):1225-1233. doi:10.1053/j.gastro.2008.12.047

21. Rolf S, Boldt LH, Parwani AS, et al. Findings and outcome of fluoroscopic visualization of the oesophageal course during catheter ablation of atrial fibrillation. EP Europace. 2011;13(6):796-802. doi:10.1093/europace/eur046

22. Piorkowski C, Hindricks G, Schreiber D, et al. Electroanatomic reconstruction of the left atrium, pulmonary veins, and esophagus compared with the "true anatomy" on multislice computed tomography in patients undergoing catheter ablation of atrial fibrillation. Heart Rhythm. 2006;3(3):317-327. doi:10.1016/j.hrthm.2005.11.027

23. Stárek Z, Lehar F, Jež J, et al. Long-term mobility of the esophagus in patients undergoing catheter ablation of atrial fibrillation: data from computer tomography and 3D rotational angiography of the left atrium. J Interv Card Electrophysiol. 2016;46(2):81-87. doi:10.1007/s10840016-0121-X

24. Musat D, Aziz EF, Koneru J, et al. Computational method to predict esophageal temperature elevations during pulmonary vein isolation. Pacing Clin Electrophysiol. 2010;33(10):1239-1248. doi:10.1111/j.1540-8159.2010.02804.x

25. Neven K, Metzner A, Schmidt B, et al. Balloon Catheter Position and its Relationship with Esophageal Temperature during Pulmonary Vein Isolation using High-Intensity Focused Ultrasound. Indian Pacing Electrophysiol J. 2012;12(5):192-203. doi:10.1016/s0972-6292(16)30542-3

26. Martinek M, Meyer C, Hassanein S, et al. Identification of a high-risk population for esophageal injury during radiofrequency catheter ablation of atrial fibrillation: procedural and anatomical considerations. Heart Rhythm. 2010;7(9):1224-1230. doi:10.1016/j.hrthm.2010.02.027

27. Matsumoto $\mathrm{Y}$, Kaneshiro T, Hijioka N, et al. Predicting factors of transmural thermal injury after cryoballoon pulmonary vein isolation. J Interv Card Electrophysiol. 2019;54(2):101-108. doi:10.1007/s10840-018-0454-8

28. Müller P, Dietrich J-W, Halbfass P, et al. Higher incidence of esophageal lesions after ablation of atrial fibrillation related to the use of esophageal temperature probes. Heart Rhythm. 2015;12(7):1464-1469. doi:10.1016/j.hrthm.2015.04.005

29. Knecht S, Sticherling C, Reichlin T, et al. Reliability of luminal oesophageal temperature monitoring during radiofrequency ablation of atrial fibrillation: insights from probe visualization and oesophageal reconstruction using magnetic resonance imaging. EP Europace. 2016;24(1):euw129. doi:10.1093/europace/euw129

30. Tschabrunn CM, Silverstein J, Berzin T, et al. Comparison between single- and multi-sensor oesophageal temperature probes during atrial fibrillation ablation: thermodynamic 
characteristics. EP Europace. 2015;17(6):891-897. doi:10.1093/europace/euu356

31. Deiss S, Metzner A, Ouyang F, et al. Incidence of Significant Delayed Esophageal Temperature Drop After Cryoballoon-Based Pulmonary Vein Isolation. J Cardiovasc Electrophysiol.

2016;27(8):913-917. doi:10.1111/jce.13008

32. Lang RM, Badano LP, Mor-Avi V, et al. Recommendations for Cardiac Chamber Quantification by Echocardiography in Adults: An Update from the American Society of Echocardiography and the European Association of Cardiovascular Imaging. J Am Soc Echocardiogr. 2015;28:1-39.e14. doi:10.1016/j.echo.2014.10.003 
CHAPTER 6 


\section{USE OF THREE-DIMENSIONAL COMPUTED TOMOGRAPHY OVERLAY FOR REAL-TIME CRYOBALLOON ABLATION IN ATRIAL FIBRILLATION REDUCES RADIATION DOSE AND CONTRAST DYE}

Bob Oude Velthuis, Marleen MD Molenaar, HG Reinhart Dorman, Y Jeroen Stevenhagen, Marcoen F Scholten, Job AM van der Palen, Jurren M van Opstal

Netherlands Heart Journal. 2017: 25 (6), 388-393 


\section{Abstract}

Aims Cryoballoon pulmonary vein (PV) isolation in patients with atrial fibrillation has proven to be effective in short-term and long-term follow-up. To visualise the PV anatomy, pre-ablation contrast pulmonary venography is commonly performed. Three-dimensional (3D) computed tomography (CT) overlay is a new technique creating a live 3D image of the left atrium by integrating a previously obtained CT scan during fluoroscopy. To evaluate the benefits of 3D CT overlay during cryoballoon ablation, we studied the use of 3D CT overlay versus contrast pulmonary venography in a randomised fashion in patients with paroxysmal atrial fibrillation undergoing cryoballoon PV isolation.

Methods and results Between October 2012 and June 2013, 30 patients accepted for PV isolation were randomised to cryoballoon PV isolation using either 3D CT overlay or contrast pulmonary venography. All patients underwent a pre-procedural cardiac CT for evaluation of the anatomy of the left atrium (LA) and the PVs. In the 3D CT overlay group, a 3D reconstruction of the LA and PVs was made. An overlay of the CT reconstruction was then projected over live fluoroscopy. Patients in the contrast pulmonary venography group received significantly more contrast agent (77.1 \pm 21.2 cc vs $40.1 \pm 17.6 \mathrm{cc}, p<0.001)$ and radiation (43.0 \pm 21.9 Gy.cm2 vs $28.41 \pm$ 11.7 Gy.cm2, $p=0.04$ ) than subjects in the 3D CT overlay group. There was no difference in total procedure time, fluoroscopy time and the amount of cryoapplications between the two groups.

Conclusion The use of 3D CT overlay decreases radiation and contrast dye exposure and can assist in guiding cryoballoon-based PV isolation. 


\section{Introduction}

Atrial fibrillation (AF) is the most common arrhythmia, affecting approximately 1.5$2 \%$ of the general population'. In 1998, the pulmonary veins (PVs) were identified as potential targets for the invasive treatment of $\mathrm{AF}^{2}$.

PV isolation is an effective treatment for patients with symptomatic paroxysmal AF and recommended in international guidelines'. Ablation success rates at 12 months range between 66 and $89 \%^{3-5}$. However, radiofrequency ablation requires tedious point-to-point delivery of multiple applications to isolate $\mathrm{PVS}^{6}$. As a result, more circular ablation catheters have been developed facilitating PV isolation. Ablation using a cryoballoon has been proven effective in short and long-term follow-up, with equal efficacy and safety, compared with the conventional radiofrequency approach ${ }^{6-10}$.

Pre-procedural contrast pulmonary venography is commonly performed to visualise the PV anatomy. Fluoroscopy is inherently associated with significant radiation exposure for both the patient and operator. Improved visualisation using iodinebased contrast agents is dose-dependent related to acute and chronic kidney failure. Several techniques have been evaluated to optimise the visualisation during the cryoablation procedure, such as the use of real-time three-dimensional oesophageal echocardiography ${ }^{11}$.

Three-dimensional (3D) computed tomography (CT) overlay creates a live 3D image during the procedure by integrating fluoroscopy with a previous CT scan or newly taken rotational angiographic 3D images of the left atrium (LA) and it has been shown to assist in radiofrequency-based PV isolation ${ }^{12}$. 3D CT overlay can facilitate optimal positioning of the cryoballoon and reduce contrast medium use and radiation exposure. Better positioning of the balloon can decrease the necessity for additional application(s) during PV isolation, using extra balloon or catheter cryo applications to achieve PV isolation. To evaluate the benefits of 3D CT overlay during cryoballoon ablation, we studied the use of 3D CT overlay versus contrast pulmonary venography in a randomised fashion in consecutive patients with paroxysmal AF undergoing cryoballoon PV isolation.

\section{Methods}

Patients were eligible for enrolment in the study when they were accepted for percutaneous PV isolation for paroxysmal AF, as defined in the current guidelines'. The exclusion criteria were as follows: (1) Patients with persistent AF, as defined in 
the current guidelines ${ }^{13}$; (2) A left atrial diameter of more than $50 \mathrm{~mm}$ in the parasternal long axis on transthoracic echocardiography; (3) Previous pulmonary vein isolation ablation (epicardial or endocardial); (4) Previous cardiac surgery; (5) Significant valvular disease present on echo (mitral or aortic valve regurgitation above grade 2, moderate to severe mitral or aortic stenosis); (6) Concomitant cardiac surgery needed; (7) Left ventricular ejection fraction <40\%; (8) Hypertrophic (obstructive) cardiomyopathy or dilated cardiomyopathy defined as an ejection fraction <40\%; (9) Pregnancy; (10) Myocardial infarction within the previous 3 months; (11) AF secondary to electrolyte imbalance, thyroid disease, other reversible or non-cardiovascular causes for AF. Patients were recruited in the outpatient clinic and included in the study after signed informed consent was obtained.

After inclusion, patients were randomised to cryoballoon PV isolation using 3D CT overlay or contrast pulmonary venography. All patients underwent a pre-procedural cardiac CT for evaluation of the anatomy of the LA and the PVs. If the patient's PV anatomy was not suitable for cryoballoon ablation, the patient was excluded. Patients were also excluded if the PVs could not be isolated with the cryoballoon alone during the procedure.

\section{Sample size calculation}

To reach statistical significance of $20 \%$ difference between the two groups regarding fluoroscopy time and contrast medium, we used the following parameters for power calculation: alpha $=5 \%$; power $=80 \%$; and assuming equal standard deviations in each group. Requiring 47 patients for fluoroscopy time and 17 for contrast medium. An interim analysis would be performed after 30 inclusions in consultation with the medical research ethics committee.

\section{Pre-procedural cardiac CT}

The pre-procedural contrast-enhanced cardiac CT was performed using a 64 multislice scanner (Toshiba Aquillion 64, Tokyo, Japan). Images were obtained at $120 \mathrm{kV}$ and $300 \mathrm{mAs}$. Rotation time was $0.4 \mathrm{~ms}$. The thickness of the reconstructed image slices was $0.3 \mathrm{~mm}$. During a 20-second end/expiratory breath hold, $80 \mathrm{~mL}$ contrast (Visipaque 320, GE Healthcare A.S., Oslo, Norway) was injected. An ECG-triggered scan was timed at $50 \%$ of the average inter-beat (RR) interval.

\section{Ablation procedure}

Two electrophysiologists (MS and JvO) with extensive experience in cryoballoon ablation performed the procedures. All patients were on oral vitamin-K antagonists with the international normalised ratio between 2.5 and 3.5. Vitamin $\mathrm{K}$ antagonists 
were continued during the procedure ${ }^{14}$. All procedures were performed under general anaesthesia and arterial blood pressure was continuously monitored. Venous access was obtained from the right and left femoral vein. A diagnostic catheter (EP XT CS 4p, BARD Medical Inc., GA, USA) was positioned in the coronary sinus for stimulation of the LA. The LA was accessed by a transseptal puncture with a Brockenbrough needle monitored by intra-cardiac echocardiography (St. Jude Medical, MN, USA), first with a SL-O sheath (St. Jude Medical, MN, USA), changed over a $0.32 \mathrm{~F}$ wire to a steerable $12 \mathrm{~F}$ sheath (Flexcath, Medtronic Inc., MN, USA). During the procedure, heparin was given to achieve an activated clotting time of $>350 \mathrm{~s}$. The use of a 23 or $28 \mathrm{~mm}$ balloon was based on the PV diameters.

\section{D CT overlay group}

Before the start of the procedure, the CT images were imported into the EP Navigator workstation (Philips Medical Systems, Best, the Netherlands) to create a 3D digital reconstruction of the anatomy of the LA and the PVs as previously described ${ }^{12}$. An automated reconstruction tool was used. Manual correction tools were used for optimisation of this reconstruction. An overlay of the CT reconstruction was then applied over live fluoroscopy and registration was performed using anatomical landmarks, catheter positions and contrast boluses in both superior PVs. Validation of the correct registration was performed in anteroposterior and lateral views (Figure 1).

\section{Contrast pulmonary venography group}

After transseptal puncture, a 7 French NIH catheter (Cordis, Miami Lakes, FL, USA) was introduced and contrast was delivered to each single PV. High definition cinematographic images of the PVs were made in left and right anterior oblique projections.

\section{Procedure}

We used the Arctic Front Advance cryoballoon (Arctic Front Advance, Medtronic Inc., MN, USA). The 28-mm balloon was used in 21 patients. A multipolar catheter (Achieve, Medtronic Inc., MN, USA) was inserted through the inner lumen of the balloon to assess PV signals before, during and after the ablation and to guide the positioning of the balloon, after which the total assembly was introduced into the LA. After inflation, the cryoballoon was advanced to occlude the PV and a contrast bolus was administered to confirm total occlusion. In general, two consecutive applications were delivered for each PV, varying from 180 to $240 \mathrm{~s}$, depending on temperatures reached. During isolation of the right PVs, the right phrenic nerve was continuously stimulated by a catheter placed in the superior vena cava. If the 


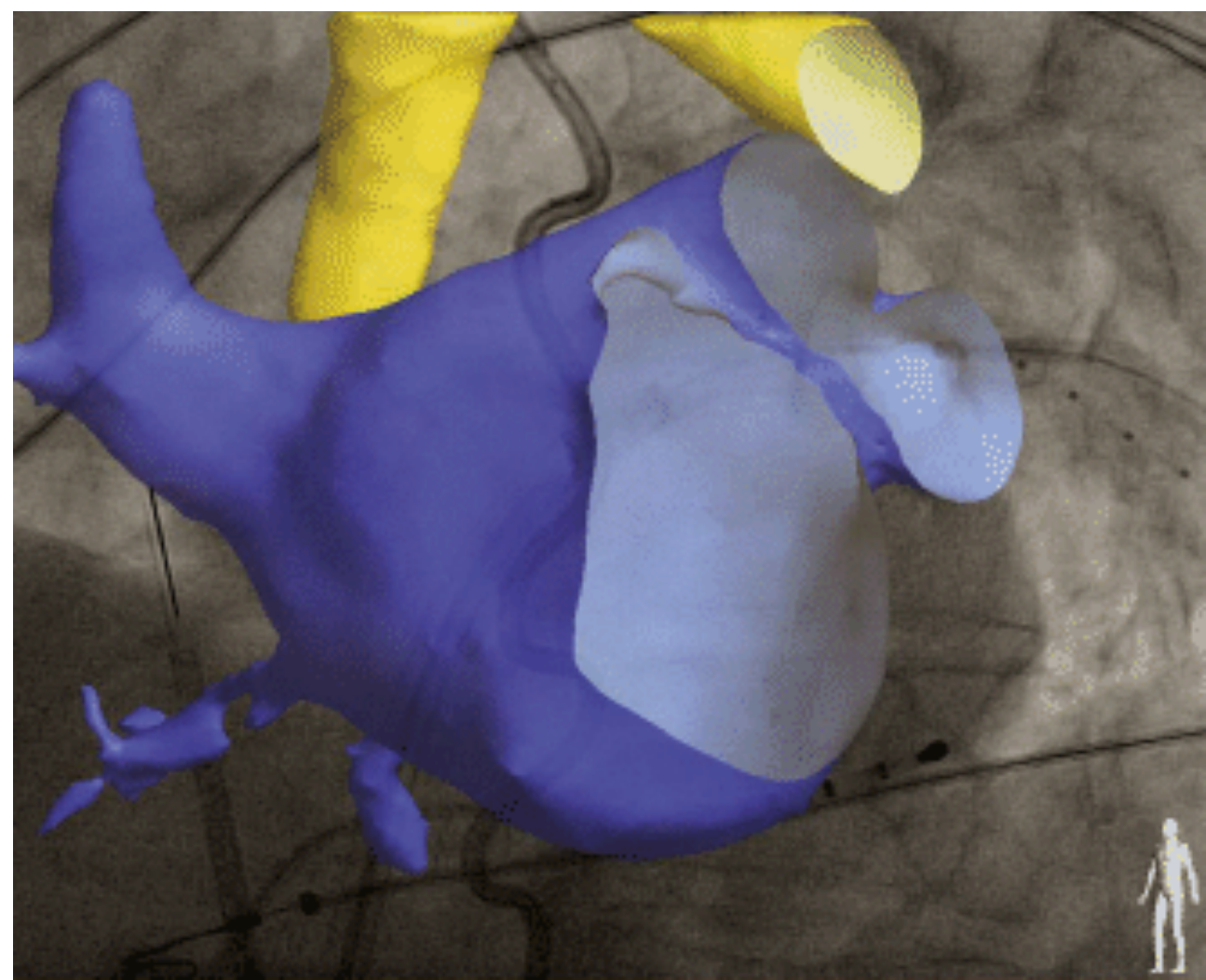

Figure 1 Example of a left atrial 3D CT overlay on the live fluoroscopy. The Achieve catheter is inserted into the left superior PV. A 4-pole catheter is placed in the coronary sinus. 
diaphragm excursions diminished during cryoablation, the ablation was immediately stopped using the double stop technique ${ }^{15}$. Bidirectional block was confirmed for each vein. After a 30-minute waiting period, all four PVs were checked for reconduction and adenosine was administered to reveal dormant PV potentials.

\section{Radiation dose}

Effective dose is a convenient quantity to estimate the stochastic risk of radiation applied to patients in interventional procedures ${ }^{16}$. Radiation of cardiac CT was measured as dose length product. The dose length product was converted to the effective dose by using a conversion factor of $0.014 \mathrm{mSv}$ Gy $1 \mathrm{~cm}^{17}$. Radiation of fluoroscopy was measured in dose area product (DAP). The DAP was converted to the effective dose by using a conversion coefficient of $0.188 \mathrm{mSv}$ Gy $1 \mathrm{~cm} 2^{18}$. For the 3D CT overlay group, the main effective dose was calculated by adding the previously mentioned conversion indices for the cardiac CT and radiation of fluoroscopy.

\section{Procedure time, number of applications, radiation exposure and contrast agent}

The total procedure time was defined as the total time between the first puncture of the femoral vein and the end of the procedure. The following were documented: (1) The time from the first puncture of the femoral vein to the transseptal puncture; (2) The total number of cryoballoon applications; (3) The total fluoroscopy time; (4) The total radiation dose; and (5) The total amount of contrast agent used. We registered the total amount of contrast used for any kind of image enhancement and for determination of the grade of occlusion during cryoablation. We therefore reported contrast usage in both groups for any reason other than visualising the PVs. In the pulmonary venography group, the amount of contrast agent used for PV visualisation alone was also registered.

\section{Follow-up}

After the intervention, patients were scheduled for four outpatient clinic visits at 1, 3, 6 and 12 months. Antiarrhythmic drugs were withdrawn after a stabilisation period of 90 days after the initial procedure and post-procedural experienced AF burden. Prior to the outpatient clinic visits at 3, 6 and 12 months, a 7-day autotriggered event recording was performed using a Vitaphone recorder (Vitaphone $\mathrm{GmbH}$, Mannheim, Germany) $)^{19,20}$. An episode of AF is defined as an episode of at least 30 s' duration. 


\section{Statistical analysis}

Results were analysed using the SPSS 17.0 software (SPSS Inc., Chicago, IL, USA). Independent samples T-test was used for numerical normally distributed data and the $\chi 2$ test was used for categorical variables. P-value $<0.05$ was considered statistically significant.

\section{Results}

From October 2012 until June 2013, 30 patients were enrolled in the study. Two patients were excluded because PV isolation could not be completed with the cryoballoon alone and additional radiofrequency applications during the same procedure were needed to achieve PV isolation (equal distribution in both groups). Table 1 shows the baseline characteristics. Mean age was $57.3 \pm 8.9$ years. Hypertension was present in six subjects (21.4\%). Mean CHADS2-VASc score was 0.8. Mean corrected left atrial end/diastolic volume was $25.1 \pm 7.2 \mathrm{cc} / \mathrm{m} 2$. Anticoagulation was used by nine patients (32.1\%) at the time of randomisation. No significant differences were found between the two operators. Table 2 shows radiation doses and amounts of contrast. Patients in the contrast pulmonary venography group received significantly more contrast agent than subjects in the 3D CT overlay group. Furthermore, patients in the direct fluoroscopy group received more radiation, as expressed in the DAP-value. There were no differences in fluoroscopy time, number of cryo applications and total procedure time between the two groups. No statistical differences were found with $p<0.05$ using an intention-to-treat analysis involving all randomised patients.

\section{Complications}

Complications were present in three patients (10.7\%). In one patient (direct fluoroscopy group), the procedure was complicated by permanent vagal nerve injury, resulting in gastric paralysis. One patient (3D CT overlay group) had transient gastric paralysis. One patient (direct fluoroscopy group) suffered from transient right hemidiaphragm paralysis. After the occurrence of vagal nerve injuries, a thermoprobe (SensiTherm, St. Jude Medical, MN, USA) was introduced in our PV procedures to monitor oesophageal temperatures during $\mathrm{PV}$ isolation, after which this complication did not longer occur'21.

\section{Clinical outcome}

At a mean follow-up of $11.9 \pm 3.9$ months (median 12 months), the success rate without continuation of antiarrhythmic therapy was $78.6 \%$ (22 patients). No difference in recurrence of AF was detected between 3D CT overlay and the contrast 
Table 1 Baseline characteristics

\begin{tabular}{|c|c|c|c|c|}
\hline Variable & $\begin{array}{l}\text { Total } \\
n=28\end{array}$ & $\begin{array}{l}\text { 3D CT overlay } \\
n=14\end{array}$ & $\begin{array}{l}\text { Pulmonary } \\
\text { Venography } \\
n=14\end{array}$ & $P$-value \\
\hline Age (years) & $57.3 \pm 8.9$ & $58.2 \pm 7.6$ & $56.5 \pm 10.3$ & ns \\
\hline Gender (male) & $20(71.4 \%)$ & $9(64.3 \%)$ & $11(78.6 \%)$ & ns \\
\hline CHADS $_{2}$-VASc score & $0.8 \pm 1.1$ & $0.7 \pm 0.7$ & $0.9 \pm 1.4$ & ns \\
\hline Hypertension & $6(21.4 \%)$ & $3(21.4 \%)$ & $3(21.4 \%)$ & ns \\
\hline Smoking & $8(28.6 \%)$ & $1(7.1 \%)$ & $7(50.0 \%)$ & 0.011 \\
\hline Diabetes & $0(0 \%)$ & $0(0 \%)$ & $0(0 \%)$ & - \\
\hline AF family history $<65$ years & $9(32.1 \%)$ & $3(21.4 \%)$ & $6(42.9 \%)$ & ns \\
\hline CAD & $0(0 \%)$ & $0(0 \%)$ & $0(0 \%)$ & - \\
\hline $\mathrm{CHF}$ & $0(0.0 \%)$ & $0(0.0 \%)$ & $0(0.0 \%)$ & - \\
\hline Endurance sports & $3(10.7 \%)$ & $2(14.3 \%)$ & $1(7.1 \%)$ & ns \\
\hline OSAS & $1(3.6 \%)$ & $0(0.0 \%)$ & $1(7.1 \%)$ & ns \\
\hline TIA/CVA & $1(3.6 \%)$ & $0(0.0 \%)$ & $1(7.1 \%)$ & ns \\
\hline Flutter & $3(10.7 \%)$ & $2(14.3 \%)$ & $1(7.1 \%)$ & ns \\
\hline Anticoagulants & $9(32.1 \%)$ & $6(42.9 \%)$ & $3(21.4 \%)$ & ns \\
\hline No ECV & $1.2 \pm 1.9$ & $1.0 \pm 1.7$ & $1.4 \pm 2.1$ & ns \\
\hline BMI & $26.8 \pm 3.0$ & $26.3 \pm 2.6$ & $27.2 \pm 3.4$ & ns \\
\hline LA volume $\left(\mathrm{cc} / \mathrm{m}^{2}\right)$ & $25.1 \pm 7.2$ & $25.1 \pm 6.2$ & $25.1 \pm 8.3$ & ns \\
\hline
\end{tabular}

Data are expressed in mean \pm SD or absolute number and percentage

AF Atrial fibrillation, CAD Coronary artery disease, CHF Congestive heart failure (Ejection Fraction $<40 \%)$, OSAS Obstructive sleep apnoea syndrome, TIA Transient ischaemic attack, CVA Cerebral vascular attack, ECV Electrical cardioversion, BMI Body mass index, LA Left atrium, NS Not significant

Table 2 PVI procedure results

\begin{tabular}{lllll}
\hline Variable & Total & 3D CT overlay & $\begin{array}{l}\text { Pulmonary } \\
\text { Venography } \\
\mathrm{n}=14\end{array}$ & P-value \\
& $\mathrm{n}=28$ & $\mathrm{n}=14$ & $77.1( \pm 21.2)$ & $<0.001$ \\
\hline Total Contrast used (cc) & $58.6( \pm 26.9)$ & $40.1( \pm 17.6)$ & $33.4( \pm 13.8)$ & $\mathrm{ns}$ \\
\hline - Without PV angio & $36.8( \pm 15.9)$ & $40.1( \pm 17.6)$ & $43.0( \pm 21.9)$ & 0.04 \\
\hline DAP $\left(G y . \mathrm{cm}^{2}\right)$ & $35.69( \pm 18.8)$ & $28.41( \pm 11.7)$ & $8.08( \pm 4.1)$ & 0.04 \\
\hline Effective dose procedure (mSv) & $6.71( \pm 3.5)$ & $5.34( \pm 2.2)$ & $30.1( \pm 8.4)$ & $\mathrm{ns}$ \\
\hline Fluoroscopy time (min) & $29.4( \pm 9.8)$ & $28.8( \pm 11.2)$ & $18.2( \pm 11.3)$ & $\mathrm{ns}$ \\
\hline Needle-TSP (min) & $16.5( \pm 8.4)$ & $14.7( \pm 3.6)$ & $106.9( \pm 17.8)$ & $\mathrm{ns}$ \\
\hline Procedure time (min) & $110.1( \pm 28.5)$ & $113.2( \pm 36.7)$ & $8.7( \pm 1.3)$ & $\mathrm{ns}$ \\
\hline Cryo-applications per procedure & $9.3( \pm 2.2)$ & $9.9( \pm 2.7)$ & &
\end{tabular}

Data are expressed in mean \pm SD

PV angio Pulmonary vein angiography, DAP Dose area product, TSP Transseptal puncture, NS Not significant 
group (12 vs $10 p=0.55$ ). One patient was lost to follow-up. Four patients were treated in a second procedure using radiofrequency ablation with touch-up of isolation gaps. Successful isolation of all PVs could not be reached in 1 patient, for which a successful video-assisted thoracoscopy PV isolation was performed. Prolonged follow-up for $9.6 \pm 3.5$ months (median 10 months) showed no recurrence.

\section{Discussion}

This study shows the feasibility of $3 D$ CT overlay in PV isolation using the cryoballoon and demonstrates significantly less radiation and use of contrast in comparison to contrast pulmonary venography. The use of 3D CT overlay has several benefits over direct fluoroscopy. As the 3D CT overlay image supplies a 3D navigation map, manoeuvring and placing the cryoballoon becomes more straightforward. This has also been demonstrated for 3D transoesophageal echocardiography ${ }^{11}$. The relative difference in radiation dose and radiation time can be explained by the fact that, for cinematographic images of the PV angiograms, a higher resolution was needed.

Contrast-induced acute kidney injury is an important complication of the use of iodinated contrast media, which accounts for a significant number of cases of hospital-acquired acute kidney injury ${ }^{22}$. As PV isolation is increasingly becoming standard therapy for patients with AF, it is essential to optimise patient safety. We realise that a CT scan requires radiation and contrast agent as well. In our study population, a pre-procedural CT scan was performed on all subjects. The amount of contrast agent (Visipaque 320) was 80 or $90 \mathrm{cc}$. Main dose length product of the CT scan in our study population was $841.3 \pm 255.5 \mathrm{mGy} . \mathrm{cm}$. Using the conversion factor of $0.014 \mathrm{mSv}$ Gy $1 \mathrm{~cm}^{17}$, this results in a main effective dose of $11.8 \mathrm{mSv}$. The combined effective dose in the 3D CT overlay group is still lower in comparison to that of the direct fluoroscopy group.

However, ideally the radiation and contrast agent necessary for the CT scan is avoided as well. This can be done by using a 3D overlay from magnetic resonance imaging (MRI). Recently, we successfully used a 3D MRI overlay in PV isolation with the cryoballoon in two patients. Although MRI was not used in this study, it could replace the $\mathrm{CT}$ scan for the $3 \mathrm{D}$ overlay and even further reduce total radiation and contrast dye exposure.

Complications were present in three (10.7\%) patients, two minor complications and one major. In all three cases, the $23-\mathrm{mm}$ balloon was used. Andrade et al. demonstrated that the smaller balloon is associated with a high incidence of 
phrenic nerve palsy? We report two cases of vagal nerve injury. Kuwahara et al. reported peri-oesophageal nerve injury as a complication of PV isolation with radiofrequency energy in 11/3695 patients ${ }^{23}$. Recent reports also demonstrate an increased incidence of oesophageal thermal lesions using the second-generation 28-mm cryoballoon ${ }^{24}$. We introduced continuous measurement of luminal oesophageal temperature after this complication, after which no vagal nerve injuries occurred. The study was terminated prematurely due to a significant difference in radiation dosage at interim analysis. Therefore, the study was underpowered with regard to the contrast dosage determined by our initial power analysis.

\section{Conclusion}

The use of 3D CT overlay has several benefits over direct fluoroscopy. As the 3D CT overlay image supplies a 3D navigation map, manoeuvring and placing the cryoballoon becomes more straightforward.

The present study shows that the use of 3D CT overlay has the potential to reduce radiation dose and exposure to contrast dye in cryoballoon-based PV isolation. Even though the study population is limited, the results show a clear benefit of this technique. We expect an additional advantage of 3D MRI overlay with respect to radiation dose. More research into this technique is necessary and will be effectuated. 


\section{References}

1. Camm AJ, Lip GGYH, De Caterina R, et al. 2012 Focused update of the ESC Guidelines for the management of atrial fibrillation: an update of the 2010 ESC Guidelines for the management of atrial fibrillation. Developed with the special contribution of the European Heart Rhythm Association. Eur Heart J. 2012;33(10):2719-2747. doi:10.1093/eurheartj/ehs253

2. Haïssaguerre $M$, Jaïs $P$, Shah DC, et al. Spontaneous Initiation of Atrial Fibrillation by Ectopic Beats Originating in the Pulmonary Veins. N Engl J Med. 1998;339(10):659-666. doi:10.1056/NEJM199809033391003

3. Cosedis Nielsen J, Johannessen A, Raatikainen $P$, et al. Radiofrequency Ablation as Initial Therapy in Paroxysmal Atrial Fibrillation. N Engl J Med. 2012;367(17):1587-1595. doi:10.1056/NEJMoa1113566

4. Jaïs $P$, Cauchemez B, Macle $L$, et al. Catheter ablation versus antiarrhythmic drugs for atrial fibrillation: the A4 study. Circulation. 2008;118(24):2498-2505. doi:10.1161/CIRCULATIONAHA.108.772582

5. Wilber DJ, Pappone C, Neuzil P, et al. Comparison of antiarrhythmic drug therapy and radiofrequency catheter ablation in patients with paroxysmal atrial fibrillation: $A$ randomized controlled trial. JAMA - J Am Med Assoc. 2010;303(4):333-340. doi:10.1001/jama.2009.2029

6. Packer DL, Kowal RC, Wheelan KR, et al. Cryoballoon ablation of pulmonary veins for paroxysmal atrial fibrillation: first results of the North American Arctic Front (STOP AF) pivotal trial. J Am Coll Cardiol. 2013;61(16):1713-1723. doi:10.1016/j.jacc.2012.11.064

7. Andrade JG, Khairy P, Guerra PG, et al. Efficacy and safety of cryoballoon ablation for atrial fibrillation: A systematic review of published studies. Heart Rhythm. 2011;8(9):1444-1451. doi:10.1016/j.hrthm.2011.03.050

8. Kuck K-HH, Brugada J, Fürnkranz A, et al. Cryoballoon or Radiofrequency Ablation for Paroxysmal Atrial Fibrillation. N Engl J Med. 2016;374(23):2235-2245. doi:10.1056/NEJMoa1602014

9. Sarabanda A V., Bunch TJ, Johnson SB, et al. Efficacy and safety of circumferential pulmonary vein isolation using a novel cryothermal balloon ablation system. J Am Coll Cardiol. 2005;46(10):1902-1912. doi:10.1016/j.jacc.2005.07.046

10. Schmidt M, Dorwarth $U$, Andresen D, et al. Cryoballoon versus RF ablation in paroxysmal atrial fibrillation: Results from the German Ablation Registry. J Cardiovasc Electrophysiol. 2014;25(1):1-7. doi:10.1111/jce.12267

11. Ottaviano L, Chierchia GB, Bregasi A, et al. Cryoballoon ablation for atrial fibrillation guided by real-time three-dimensional transoesophageal echocardiography: A feasibility study. EP Europace. 2013;15(7):944-950. doi:10.1093/europace/eus431

12. Stevenhagen J, Van Der Voort PH, Dekker LRC, et al. Three-dimensional CT overlay in comparison to CartoMerge for pulmonary vein antrum isolation. J Cardiovasc Electrophysiol. 2010;21(6):634639. doi:10.1111/j.1540-8167.2009.01665.x

13. Camm AJ, Kirchhof P, Lip GYH, et al. Guidelines for the management of atrial fibrillation: the Task Force for the Management of Atrial Fibrillation of the European Society of Cardiology (ESC). Europace. 2010;12(10):1360-1420. doi:10.1093/europace/euq350

14. Velthuis BO, Stevenhagen J, Van Opstal JM, et al. Continuation of vitamin K antagonists as acceptable anticoagulation regimen in patients undergoing pulmonary vein isolation. Neth Heart J. 2012;20(1):12-15. doi:10.1007/s12471-011-0223-0

15. Andrade JG, Dubuc M, Guerra PG, et al. Pulmonary Vein Isolation Using a Second-Generation Cryoballoon Catheter: A Randomized Comparison of Ablation Duration and Method of Deflation. J 
Cardiovasc Electrophysiol. 2013;24(6):692-698. doi:10.1111/jce.12114

16. FW S, J Z. Dose Conversion Coefficients for Interventional Procedures. Radiat Prot Dosimetry. 2005;117(1-3). doi:10.1093/RPD/NCI753

17. Karaarslan E, Ulus S, Bavbek C. Estimated radiation dose and image quality comparison of the scan protocols in dual-source computed tomography coronary angiography. J Med Imaging Radiat Oncol. 2013;57(4):407-414. doi:10.1111/1754-9485.12036

18. Ector J, Dragusin O, Adriaenssens B, et al. Obesity Is a Major Determinant of Radiation Dose in Patients Undergoing Pulmonary Vein Isolation for Atrial Fibrillation. J Am Coll Cardiol. 2007;50(3):234-242. doi:10.1016/j.jacc.2007.03.040

19. Müller A, Scharner W, Borchardt T, et al. Reliability of an external loop recorder for automatic recognition and transtelephonic ECG transmission of atrial fibrillation. J Telemed Telecare. 2009;15(8):391-396. doi:10.1258/jtt.2009.090402

20. Velthuis BO, Bos J, Kraaier K, et al. Performance of an External Transtelephonic Loop Recorder for Automated Detection of Paroxysmal Atrial Fibrillation. Ann Noninvasive Electrocardiol. 2013;18(6):564-570. doi:10.1111/anec.12075

21. Molenaar MMD, Scholten MF, Hesselink T, et al. High incidence of low esophageal temperatures during cryoballoon therapy for atrial fibrillation. In: Heart Rythm Society. Boston; 2014.

22. McCullough PA. Contrast-Induced Acute Kidney Injury. J Am Coll Cardiol. 2008;51(15):1419-1428. doi:10.1016/j.jacc.2007.12.035

23. Kuwahara T, Takahashi A, Takahashi Y, et al. Clinical characteristics and management of periesophageal vagal nerve injury complicating left atrial ablation of atrial fibrillation: lessons from eleven cases. J Cardiovasc Electrophysiol. 2013;24(8):847-851. doi:10.1111/jce.12130

24. Metzner A, Burchard A, Wohlmuth $P$, et al. Increased incidence of esophageal thermal lesions using the second-generation 28-mm cryoballoon. Circ Arrhythm Electrophysiol. 2013;6(4):769-775. doi:10.1161/CIRCEP.113.000228 
CHAPTER 7 


\section{PREVALENCE AND CONSEQUENCES OF INCIDENTAL FINDINGS DETECTED BY COMPUTED TOMOGRAPHY PRIOR TO PULMONARY VEIN ISOLATION OR TRANSCATHETER AORTIC VALVE REPLACEMENT}

Tim Hesselink, Marleen MD Molenaar, Loes FA Scholten, Matthijs FL Meijs, Roel Slenter, Martin G Stoel, Jurren M Van Opstal, Gert Van Houwelingen, Marcoen F Scholten 


\section{Abstract}

Purpose: Aim of the study was to investigate the prevalence and consequences of incidental findings detected by computed tomography (CT) in different cardiac populations.

Methods: All consecutive patients in which a pre-procedural CT for pulmonary vein isolation (PVI) $(\mathrm{N}=396)$ or trans aortic valve replacement (TAVR) ( $N=142)$ was performed were included in this single center retrospective study. The CT scans were analyzed by an experienced radiologist and incidental findings were identified from the report. Medical records were reviewed to identify the additional diagnostic procedures or therapies.

Results: A total of 84 incidental findings was found in 79/396 (20\%) PVI patients and 146 in 95/142 (67\%) TAVR patients, predominantly vascular findings. Compared to patients without incidental findings, PVI patients with incidental findings were significantly older (59.8 SD 8.7 vs. 55.8 SD 10.6 years, $p<0.01$ ) and more often had hypertension ( $47 \%$ vs. $30 \%, p<0.01$ ), TAVR patients with incidental findings more often had COPD (26\% vs. 11\%, $\mathrm{p}=0.016$ ). A total of 71/396 (18\%) PVI patients and 52/142 (37\%) TAVR patients had additional diagnostic procedures. In 31/396 (8\%) PVI patients and 9/142 (6\%) TAVR patients therapy was initiated because of these findings.

Conclusions: Incidental findings on the pre-procedural CT scan are common both in patients undergoing PVI and TAVR screening. Additional diagnostic procedures are needed in $18 \%$ of PVI patients and $37 \%$ of TAVR patients, resulting in $8 \%$ of PVI patients and $6 \%$ of TAVR patients undergoing therapy. Anxiety could be reduced by actively informing patients that although the chance of an incidental finding is present, only a small percentage requires (invasive) therapy. 


\section{Introduction}

Pre-procedural computed tomography (CT) facilitates both the pulmonary vein isolation (PVI) procedure in the treatment of atrial fibrillation and the transcatheter aortic valve replacement (TAVR) procedure in the treatment of severe aortic stenosis by providing detailed anatomical information ${ }^{1,2}$.

In addition to the requested information, incidental findings that identify potential pathologies may be present on the $\mathrm{CT}$ images. Presence of these incidental findings is very common in both the PVI and TAVR population ${ }^{3-14}$. However, there is paucity in recommendations on the required clinical consequences of incidental findings in these populations.

As a result of the improvement of imaging resolutions and the growing number of $\mathrm{PVI}$ and TAVR procedures performed, the prevalence of incidental findings increases. Therefore recommendations on how to manage incidental findings becomes more and more essential. This study aims to contribute to these recommendations by assessment of the implications of incidental findings. Therefore we investigated both prevalence and subsequent consequences of incidental findings detected by $\mathrm{CT}$ in both a PVI and TAVR population.

\section{Methods}

\section{Study Population}

All consecutive patients in which a pre-procedural CT for PVI or TAVR was performed between December 2012 and December 2015 at the Medisch Spectrum Twente (Enschede, the Netherlands) were included in this single center retrospective study. In all patients accepted for TAVR, transoesophegeal echocardiography and coronary angiography have been performed as a part of the TAVR screening. Need for informed consent for this retrospective study was waived by the medical ethical committee and this study complies with the Declaration of Helsinki.

\section{Cardiac CT PVI protocol}

Image acquisition by cardiac CT angiography was performed on a 64 detector Toshiba Aquilion CT scanner (Toshiba Medical Systems, Tokyo, Japan). CT scanning was synchronized with the electrocardiogram if possible (76\% of PVI patients). Parameters were field of view (FOV) 500 mm, 120 kV, 300 mAs (automatically adjusted to the cardiac cycle); typically at $75 \%$ of the cardiac cycle. Acquisition was performed using a slice thickness of $0.5 \mathrm{~mm}$ and slice interval $0.3 \mathrm{~mm}$. Breath hold was practiced before scanning combined with the ECG. During the CT a bolus of 
$80 \mathrm{~mL}$ iodinated contrast agent (Visipaque $320 \mathrm{mg} \mathrm{I} / \mathrm{mL}$ ) was injected at the antecubital vein at a speed of $3 \mathrm{~mL} / \mathrm{s}$ followed by saline chaser of $20 \mathrm{~mL}$. The chaser is used to make sure the total amount of injected contrast circulates as an entire bolus through the heart. In this way the best contrast opacification is obtained with the lowest possible amount of contrast agent. Bolus tracking was performed until a region of interest $(\mathrm{ROI})$ of $>170$ Hounsfield Units was measured in the left atrium.

\section{Cardiac CT TAVR protocol}

Image acquisition by cardiac CT angiography was performed on a 64 detector Toshiba Aquilion CT scanner (Toshiba Medical Systems, Tokyo, Japan). Scan parameters were FOV 500mm, $120 \mathrm{kV}$. Body mass and weight-adjusted mAs was typically around 200-300mAs. The scanogram was made from the aortic knob until below the trochanter minor with a second point of interest from the aortic knob until the level of the diaphragm to scan the heart and thoracic aorta. This cardiac CT was also retrospectively synchronized with the electrocardiogram at $75 \%$ of the cardiac cycle. Slice thickness and slice interval were $0.5 \mathrm{~mm}$ and $0.3 \mathrm{~mm}$. Bolus tracking was performed to reach a ROI >170 Hounsfield Units afterwards the cardiac CT was made, directly followed by a CT abdominal aorta from the diaphragm until the trochanter minor. Since the longer scanning distance a $90 \mathrm{~mL}$ contrast agent at $3 \mathrm{~mL} / \mathrm{s}$ was used, followed by $20 \mathrm{~mL}$ saline chaser.

\section{Analysis for incidental findings}

The cardiac CT scan was analyzed by an experienced radiologist for incidental findings in the 2D axial images using Window Width (W) and Window Center (C). These settings were lung windows (1600W/-650C); mediastinal windows (350W/65C) and bone windows (2000W/400C) for PVI scans. For TAVR scans an additional liver window (170W/120C) was used. Coronary calcifications were judged for severity as described by Shemesh et al. by an experienced cardiologist ${ }^{15}$. Briefly, high Shemesh calcification scores correspond with increased presence of calcifications.

\section{Incidental findings and consequences}

We defined incidental findings from the radiology report as those not previously identified and potentially requiring either additional diagnostic evaluation or treatment. Incidental findings were followed up and treated according to the applicable guidelines. If no guideline was available they were treated according to clinical judgement. Specifically nodules in the lung were followed up according to the recommendations of the Fleisher society and renal cysts according to the Bosniak classification ${ }^{16-18}$. Incidental findings with distinctive benign signs were not 
Table 1 Baseline characteristics PVI patients ( $n=396)$

\begin{tabular}{llll}
\hline Patient characteristics & Incidental finding $(\mathrm{n}=79)$ & No incidental finding $(\mathrm{n}=317)$ & $P$ \\
\hline Age (years) & $59.8 \pm 8.7$ & $55.8 \pm 10.6$ & 0.002 \\
\hline Male & $53(67 \%)$ & $212(67 \%)$ & 0.972 \\
\hline BMI & $26.7 \pm 3.1$ & $26.9 \pm 3.7$ & 0.797 \\
\hline Paroxysmal AF & $65(82 \%)$ & $273(86 \%)$ & 0.389 \\
\hline Hypertension & $37(47 \%)$ & $95(30 \%)$ & 0.004 \\
\hline Diabetes mellitus & $4(5 \%)$ & $12(4 \%)$ & 0.607 \\
\hline Prior CAD & $5(6 \%)$ & $23(7 \%)$ & 0.774 \\
\hline Prior TIA & $2(3 \%)$ & $11(5 \%)$ & 0.676 \\
\hline Prior CVA & $2(3 \%)$ & $5(2 \%)$ & 0.566 \\
\hline COPD & $5(6 \%)$ & $8(3 \%)$ & 0.194 \\
\hline LAVI $\left(c c / m^{2}\right)^{\star}$ & $30.3 \pm 8.3$ & $28.2 \pm 8.5$ & 0.097 \\
\hline CHA $_{2} \mathrm{DS}_{2}$ VASc & $1.35 \pm 1.05$ & $1.12 \pm 1.08$ & 0.081
\end{tabular}

Values are $\mathrm{n}(\%)$ or mean \pm SD (Standard deviation). *Available in 285 patients. Abbreviations: BMI; body mass index, AF; atrial fibrillation, CAD; coronary artery disease, TIA; transient ischemic attack, CVA; cerebro vascular accident, COPD; chronic obstructive pulmonary disease, LAVI; left atrial volume index.

Table 2 Baseline characteristics TAVR patients ( $n=142)$

\begin{tabular}{|c|c|c|c|}
\hline Patient characteristics & Incidental finding $(\mathrm{n}=95)$ & No incidental finding $(n=47)$ & $P$ \\
\hline Age (years) & $81.6 \pm 5.9$ & $83.2 \pm 4.5$ & 0.098 \\
\hline Male & $38(40 \%)$ & $16(34 \%)$ & 0.495 \\
\hline BMI & $27.9 \pm 5.0$ & $28.1 \pm 4.2$ & 0.737 \\
\hline Hypertension & $48(51 \%)$ & $31(66 \%)$ & 0.079 \\
\hline Dyslipidemia & $59(62 \%)$ & $26(55 \%)$ & 0.441 \\
\hline Diabetes mellitus & $26(27 \%)$ & $15(32 \%)$ & 0.577 \\
\hline Prior myocardial infarction & $11(12 \%)$ & $7(15 \%)$ & 0.580 \\
\hline Prior PCl & $25(26 \%)$ & $13(28 \%)$ & 0.866 \\
\hline Prior CABG & $20(21 \%)$ & $5(11 \%)$ & 0.095 \\
\hline Prior valve surgery & $3(3 \%)$ & $4(9 \%)$ & 0.238 \\
\hline Prior TIA & $12(13 \%)$ & $5(11 \%)$ & 0.733 \\
\hline Prior CVA & $11(12 \%)$ & $9(19 \%)$ & 0.260 \\
\hline COPD & $25(26 \%)$ & $5(11 \%)$ & 0.016 \\
\hline NYHA class & $2.54 \pm 0.65$ & $2.32 \pm 0.78$ & 0.082 \\
\hline Atrial fibrillation & $41(43 \%)$ & $17(36 \%)$ & 0.429 \\
\hline Log Euroscore I & $16.8 \pm 8.2$ & $15.1 \pm 8.8$ & 0.256 \\
\hline Log Euroscore II & $4.4 \pm 3.6$ & $3.7 \pm 2.8$ & 0.220 \\
\hline LVEF >50\% & $66(70 \%)$ & $38(81 \%)$ & 0.132 \\
\hline LVEF 30\%-50\% & $24(25 \%)$ & $8(17 \%)$ & 0.250 \\
\hline LVEF $<30 \%$ & $5(5 \%)$ & $1(2 \%)$ & 0.386 \\
\hline Peak gradient (mmHg) & $70.7 \pm 21.2$ & $71.0 \pm 23.4$ & 0.943 \\
\hline Aortic valve area $\left(\mathrm{cm}^{2}\right)$ & $0.77 \pm 0.24$ & $0.77 \pm 0.18$ & 0.942 \\
\hline
\end{tabular}

Values are $\mathrm{n}$ (\%) or mean \pm SD (Standard deviation). Abbreviations: BMl; body mass index, $\mathrm{PCl}$; percutaneous coronary intervention, CABG; coronary artery bypass grafting, TIA; transient ischemic attack, CVA; cerebro vascular accident, COPD; chronic obstructive pulmonary disease, NYHA; New York Heart Association, LVEF; left ventricular ejection fraction. 
followed up to avoid unnecessary investigations or radiation exposure. Consequences of incidental findings were identified from the electronic patient files and survival was checked in the hospitals' central database.

\section{Statistical Analysis}

All variables are reported as mean with SD (standard deviation) unless stated otherwise. Continuous variables were compared with either Student's T-test in case of normal distribution or Wilcoxon rank-sum test in case of non-normal distribution. Qualitative variables are presented as numbers and proportions and were compared using Fisher's exact test. A two-sided $p$ value of $<0.05$ is considered statistically significant. Survival was calculated using a Kaplan Meyer curve and log-rank test. Statistical analysis was performed using IBM SPSS Statistics version 22.0.

\section{Results}

\section{Patient characteristics}

In 538 patients (396 PVI and 142 TAVR patients) a pre-procedural cardiac CT was performed during the study period. Mean follow up was $14.0 \pm 8.3$ months in PVI and $7.2 \pm 7.9$ months in TAVR patients. Baseline characteristics of PVI and TAVR patients, grouped by the presence or absence of incidental findings, are shown in Tables 1 and 2.

\section{Consequences of incidental findings in PVI patients}

Incidental findings in $\mathrm{PVI}$ patients, additional diagnostic procedures and/or therapeutic consequences are shown in Table 3. 84 incidental findings were found in 79/396 (20\%) PVI patients. The group with incidental findings was significantly older ( $59.8 \pm 8.7$ vs. $55.8 \pm 10.6$ years, $p<0.01)$ and there was a significantly higher incidence of hypertension ( $47 \%$ vs. $30 \%, p<0.01$ ) when compared to the group without. In 71/396 (18\%) patients 81 additional diagnostic procedures were performed, most of them being additional CTs or coronary angiographies. Outcomes of $31 / 81$ (38\%) additional diagnostic procedures led to therapeutic consequences in 31/396 (8\%) patients. In 23/396 (6\%) patients therapeutic consequences consisted of medication or lifestyle therapy.

Additional coronary angiography led to invasive therapeutic consequences in 2 patients. In these two patients with a total Shemesh calcification score of 8 and 12 respectively a $\mathrm{PCl}$ and $\mathrm{CABG}$ combined with a maze procedure were performed. Both patients were asymptomatic at time of the primary $\mathrm{CT}$. All other additional coronary angiographies led to noninvasive therapeutic consequences like initiation of 
Table 3 Incidental findings in PVI patients ( $n=396$ )

\begin{tabular}{|c|c|c|c|c|}
\hline Pathology & $n$ & Diagnostic procedure (n) & Therapy (n) & Therapy type (n) \\
\hline \multicolumn{5}{|l|}{ Vascular } \\
\hline Coronary calcifications & 30 & $\begin{array}{l}\text { n/a (9) } \\
\text { MIBI-scan (5) } \\
\text { CAG (16) }\end{array}$ & 25 & $\begin{array}{l}\text { Medication/lifestyle (23) } \\
\text { PCI (1) } \\
\text { CABG/MAZE (1) }\end{array}$ \\
\hline Thoracic aortic dilatation & 8 & TTE or CT (8) & 0 & Conservative \\
\hline LAA trombus & 2 & TEE (2) & 2 & OAC (2) \\
\hline Signs of PHT & 1 & TTE (1) & 0 & Conservative \\
\hline Pericardial fluid & 1 & TEE (1) & 0 & Conservative \\
\hline ASD & 1 & Catheterisation (1) & 0 & Conservative \\
\hline Left isomerism & 1 & CT (1) & 1 & VATS-PVI (1) \\
\hline Abbarant CA & 1 & $\mathrm{CT}(1)$ & 1 & Cardiac surgery (1) \\
\hline \multicolumn{5}{|l|}{ Pulmonary } \\
\hline Nodules $<4 \mathrm{~mm}$ & 1 & $\mathrm{CT}(1)$ & 0 & Conservative \\
\hline Nodules 4-6mm & 14 & CT (17) & 1 & Metastatic disease (1) \\
\hline Nodules 6-8mm & 4 & CT, PET/CT (4) & 0 & Conservative \\
\hline Nodules $>8 \mathrm{~mm}$ & 3 & $\mathrm{CT}, \mathrm{PET} / \mathrm{CT}$ (4) & 0 & Conservative \\
\hline Lymphadenopathy & 7 & $\begin{array}{l}\mathrm{CT}, \mathrm{PET} / \mathrm{CT}(5) \\
\text { Biopsy (3) } \\
\mathrm{MRI}(2)\end{array}$ & 0 & Conservative \\
\hline Undetermined lesion & 5 & $\begin{array}{l}\mathrm{CT}, \mathrm{PET} / \mathrm{CT} \text { (4) } \\
\mathrm{CT} \text { planned, not performed } \\
\text { due to endocarditis ( } 1 \text { ) }\end{array}$ & 1 & Lobectomy (1) \\
\hline Interstitual lung disease & 1 & Lung function testing (1) & 0 & Conservative \\
\hline Bronchiolitis & 1 & Lung function testing (1) & 0 & Conservative \\
\hline \multicolumn{5}{|l|}{ Other } \\
\hline Elevated hemidiafragm & 1 & X-thorax (1) & 0 & Conservative \\
\hline Oesophegeal structure & 1 & Endo/echo (1) & 0 & Conservative \\
\hline Liver lesion & 1 & Echo (1) & 0 & Conservative \\
\hline Total & 84 & & 31 & \\
\hline
\end{tabular}

Abbreviations: LAA; left atrial appendage, PHT; pulmonary hypertension, ASD; Atrial septal defect, CA; coronary artery, MIBI; Methoxy Isobutyl Isonitrile, CAG; coronary angiogram, TTE; transthoracal echocardiogram, CT; computed tomography, TEE; transoesophegeal echocardiogram, PET/CT; Positron emission tomography-computed tomography, CABG; coronary artery bypass grafting. 
medication, predominantly statin treatment, and recommendations for lifestyle changes. Mean Shemesh calcification score in this noninvasive therapeutic consequences group was $2.7 \pm 2.3$.

None of the additional CTs showed primary lung carcinoma. In one patient the additional CT led to the diagnosis of metastasized prostate cancer. In one patient tissue analysis showed bronchiectasis with inflammatory changes of the cystic type mimicking lung carcinoma and therefore a lobectomy was performed. In 8 patients a dilation of the aorta between $37 \mathrm{~mm}-41 \mathrm{~mm}$ was found, this dilation was not present on earlier echocardiograms. Cardiac surgery because of malignant coronary artery course was performed in one patient.

\section{Consequences of incidental findings in TAVR patients}

Incidental findings in TAVR patients, additional diagnostic procedures and/or therapeutic consequences are shown in Table 4. 146 incidental findings were found in 95/142 (67\%) TAVR patients. In the TAVR patients, there was a significantly higher incidence of chronic obstructive pulmonary disease (COPD) in the group with incidental finding ( $26 \%$ vs. $11 \%, p=0.016$ ). Additional diagnostic procedures were performed in 52/142 (37\%) TAVR patients, being 55\% of TAVR patients with incidental findings. In 9/52 (17\%) patients additional diagnostic procedures led to therapeutic consequences.

In 6 patients (4.2\%) additional diagnostic procedures showed malignancies, all treated successfully with surgery. Several incidental findings led to delay or cancellation of TAVR, predominantly findings of lesions that were suspected for malignancy. Delay to TAVR was significantly longer ( $54.8 \pm 39.1$ vs. $30.5 \pm 25.9$ days, $p=0.014$ ) in patients with incidental findings when compared to those without. There was no significant difference in death of all causes during follow-up between patients with and without incidental findings $(p=0.800)$. 
Table 4 Incidental findings in TAVR patients ( $n=142$ )

\begin{tabular}{|c|c|c|}
\hline Incidental finding & $\mathrm{n}$ & Diagnosis and treatment (n) \\
\hline \multicolumn{3}{|l|}{ Vascular } \\
\hline Abdominal aortic dilatation & 17 & $\begin{array}{l}\text { Conservative (15) } \\
\text { Endoprosthesis (1) } \\
\text { Unknown (1) }\end{array}$ \\
\hline Thoracic aortic dilatation & 7 & Conservative (7) \\
\hline Signs of decompensation & 12 & Conservative (12) \\
\hline Signs of pulmonary hypertension & 11 & Conservative (11) \\
\hline LAA thrombus & 2 & $\begin{array}{l}\text { True thrombus, already OAC (1) } \\
\text { TEE negative (1) }\end{array}$ \\
\hline Aortic thrombus/plaque & 2 & Conservative (2) \\
\hline $\begin{array}{l}\text { Subclavian art } \\
\text { aneurysm/thrombus }\end{array}$ & 1 & Conservative (1) \\
\hline Pulmonary embolism & 1 & Embolism treated with OAC (1) \\
\hline Aneurysm artery gastro-epiploica & 1 & Small aneurysm, conservative (1) \\
\hline \multicolumn{3}{|l|}{ Pulmonary } \\
\hline Lymphadenopathy & 9 & $\begin{array}{l}\text { Conservative (8) } \\
\text { Sarcoidosis, conservative (1) }\end{array}$ \\
\hline Nodules 4-6mm & 10 & $\begin{array}{l}\text { Conservative/pending FU (7) } \\
\text { Sarcoidosis, conservative (1) } \\
\text { PET/CT negative lesion (1) } \\
\text { Unrelated death before FU (1) }\end{array}$ \\
\hline Nodules 6-8mm & 3 & Conservative/pending FU (3) \\
\hline Nodules $>8 \mathrm{~mm}$ & 5 & $\begin{array}{l}\text { Lung carcinoma, surgery (1) } \\
\text { Metastasis colon carcinoma, surgery (1) } \\
\text { Wegener, conservative (1) } \\
\text { Sarcoidosis, conservative (1) } \\
\text { Unknown (1) }\end{array}$ \\
\hline Bronchiolitis & 3 & Conservative (3) \\
\hline Lung fibrosis (severe) & 3 & Conservative (3) \\
\hline Pneumonia & 3 & Conservative (3) \\
\hline \multicolumn{3}{|l|}{ Gastrointestinal } \\
\hline Lesion pancreas & 9 & $\begin{array}{l}\text { Conservative }(7) \\
\text { Adenocarcinoma, surgery ( } 1 \text { ) } \\
\text { Neuroendocrine tumor, laparoscopic resection after } \\
\text { TAVR (1) }\end{array}$ \\
\hline Liver lesion & 7 & $\begin{array}{l}\text { Cyst, conservative (6) } \\
\text { Unknown (1) }\end{array}$ \\
\hline Oesophagus wall thickening & 2 & $\begin{array}{l}\text { Normal gastroscopy (1) } \\
\text { Unknown (1) }\end{array}$ \\
\hline Oesophagus diverticulum & 1 & Conservative (1) \\
\hline Lymfeadenopathy & 1 & Normal gastroscopy, conservative (1) \\
\hline Colon wall thickening & 1 & Conservative (1) \\
\hline
\end{tabular}




\begin{tabular}{|c|c|c|}
\hline Sigmoid lesion & 1 & Non-malignant polyp, polypectomy (1) \\
\hline Megasigmoid & 1 & Conservative (1) \\
\hline Intrathoracic stomach & 3 & Conservative (3) \\
\hline Galbladder anomaly & 2 & Unknown (2) \\
\hline \multicolumn{3}{|l|}{ Gynaecological } \\
\hline Uterus lesion & 3 & $\begin{array}{l}\text { Uterus myomatosus, conservative (2) } \\
\text { Polyp, conservative (2) }\end{array}$ \\
\hline Ovarian cyst & 2 & Conservative (2) \\
\hline Endometrium carcinoma & 1 & Endometrium carcinoma, laparoscopic hysterectomy (1) \\
\hline Adnex leasion & 1 & Dermoid cyst, conservative (1) \\
\hline \multicolumn{3}{|l|}{ Renal / Bladder } \\
\hline Renal artery origostenosis & 2 & Conservative (2) \\
\hline Renal cyst $\geq$ Bosniak 2F & 2 & Conservative (2) \\
\hline Renal infarction & 2 & Conservative (2) \\
\hline Adrenal gland node/enlargement & 5 & $\begin{array}{l}\text { Adenoma, conservative (2) } \\
\text { PET/CT negative, conservative (1) } \\
\text { Incidentaloma eci, conservative (2) }\end{array}$ \\
\hline Urine bladder thickening & 2 & $\begin{array}{l}\text { Malignancy, surgery (1) } \\
\text { Normal cystoscopy, conservative (1) }\end{array}$ \\
\hline \multicolumn{3}{|l|}{ Other } \\
\hline Multinodular struma & 3 & Conservative (3) \\
\hline Spine fractures & 2 & Conservative (2) \\
\hline Lesion groin eci & 1 & Unknown (1) \\
\hline Gynecomastia & 1 & Unknown (1) \\
\hline Spleen lesion & 1 & Unknown (1) \\
\hline Total & 146 & \\
\hline
\end{tabular}

Abbreviations: TAVR; trans aortic valve replacement LAA; left atrial appendage, OAC; oral anticoagulation, TEE; transoesophegeal echocardiogram, PET/CT; Positron emission tomography-computed tomography, CABG; coronary artery bypass grafting. 


\section{Discussion}

With increasing numbers of PVI and TAVR being performed, the prevalence and subsequent consequences of incidental findings on CT are of increasing importance. This study showed that incidental findings were present in $20 \%$ of PVI patients and $67 \%$ of TAVR patients. Incidental findings are more likely in PVI patients with higher age or hypertension and TAVR patients with COPD. 8\% of PVI patients and $6 \%$ of TAVR patients required treatment because of the incidental findings. As expected, the rate of incidental findings was lower in PVI patients when compared to TAVR patients due to perceived lower risk profile, significantly younger patients and smaller FOV scanned. Several other studies evaluating incidental CT findings reported a broad range of $23-69 \%$ incidental findings in PVI patients $\mathrm{s}^{3,4,7-9}$ and $17-58 \%$ in TAVR patients $5^{5,6,10-14}$. A higher prevalence of incidental findings in our TAVR patients group, compared to literature, might be due to a broad inclusion of several vascular findings in our study. Hussien et al. found $58 \%$ incidental findings in preprocedural CTs of TAVR patients, but did not analyze the abdomen in their study, which might have underestimated the prevalence ${ }^{14}$.

\section{Consequences of incidental findings}

Although the prevalence of incidental findings was high, the findings differed in terms of impact. Consequences ranged from incidental findings needing direct treatment, such as malignant carcinomas, to those requiring follow-up or which are of unknown significance without additional investigations. We expect the last category to become of incremental importance with the increasing number of imaging techniques being utilized in clinical practice. Beyond medical indicated imaging, patients nowadays have access to commercial full body scanning techniques. Obuchowski et al. conducted a pilot randomized controlled trial of total body screening in asymptomatic subjects and found 64\% abnormal findings requiring further evaluation, but a vast minority of them requiring treatment ${ }^{19}$. There is an open debate regarding the detrimental and beneficial effects of recognizing incidental findings in CT scans. On one hand there are uncertain clinical benefits, increased costs and radiation exposure associated with follow-up diagnostic procedures and induced anxiety for the patients. On the other hand, early detection of pathology could allow early treatment and potentially save lives. In a related field, The American Expert Consensus Document on Coronary Computed Tomographic Angiography also states that this is an area without consensus ${ }^{20}$. 


\section{Coronary calcifications in PVI patients}

Coronary calcifications were prevalent in a vast amount of PVI patients; however no formal Agatston coronary calcium scoring was performed due to the nature of the scanning technique. Visual scoring of coronary calcium revealed that patients with atrial fibrillation indeed have subclinical coronary artery disease on CT more often when compared to controls ${ }^{21}$. They also develop cardiovascular disease more often, at a younger age and in more severe forms ${ }^{22}$.

\section{Treatment of incidental findings}

We found that $8 \%$ of PVI patients and $6 \%$ of TAVR patients underwent treatment during limited follow-up. An even smaller amount of $2 \%$ of PVI required invasive therapy. So, anxiety could be reduced by actively informing patients that although the chance of an incidental finding is present, only a small percentage requires (invasive) therapy. The treatment during follow-up included a $4.2 \%$ incidence in treatment of newly discovered malignancies in TAVR patients, similar to that reported in studies by Ben Dor et al and Gufler et $\mathrm{al}^{5,10}$. Detrimental effect of an incidental finding was found in one PVI patient that underwent lobectomy because of pathology mimicking lung cancer. On the other hand, metastasis of a previously unknown cancer was also discovered in one PVI patient that underwent subsequent treatment. In TAVR patients there is conflicting evidence regarding the presence of incidental findings and overall outcome as well as mortality ${ }^{12,13,23}$. We found no association of incidental findings with death of all causes in our population. However, several findings delayed the TAVR procedure or required treatment.

\section{Limitations}

We recognize our study has limitations because of the retrospective design. Although follow-up was limited, we found that a significant percentage of incidental findings required treatment or altered scheduled treatment.

\section{Conclusion}

Incidental findings on the pre-procedural CT scan are common both in patients undergoing PVI and TAVR screening. Additional diagnostic procedures were needed in $18 \%$ of PVI patients and $37 \%$ of TAVR patients, resulting in therapeutic consequences in $8 \%$ of $\mathrm{PVI}$ patients and $6 \%$ of TAVR patients. Anxiety could be reduced by actively informing patients that although the chance of an incidental finding is present, only a small percentage requires (invasive) therapy. 


\section{References}

1. Pontone G, Andreini D, Bartorelli AL, et al. Feasibility and accuracy of a comprehensive multidetector computed tomography acquisition for patients referred for balloon-expandable transcatheter aortic valve implantation. Am Heart J. 2011. doi:10.1016/j.ahj.2011.03.003

2. Calkins H, Kuck KH, Cappato R, et al. 2012 HRS/EHRA/ECAS expert consensus statement on catheter and surgical ablation of atrial fibrillation: recommendations for patient selection, procedural techniques, patient management and follow-up, definitions, endpoints, and research trial design. J Interv Card Electrophysiol. 2012;33(2):171-257. doi:10.1007/s10840-012-9672-7

3. Martins RP, Muresan L, Sellal J-M, et al. Incidental extracardiac findings in cardiac computed tomography performed before radiofrequency ablation of atrial fibrillation. Pacing Clin Electrophysiol. 2011;34(12):1665-1670. doi:10.1111/j.1540-8159.2011.03194.x

4. Casella M, Perna F, Pontone G, et al. Prevalence and clinical significance of collateral findings detected by chest computed tomography in patients undergoing atrial fibrillation ablation. EP Europace. 2012. doi:10.1093/europace/eur300

5. Gufler H, Schulze CG, Wagner S. Incidental findings in computed tomographic angiography for planning percutaneous aortic valve replacement: advanced age, increased cancer prevalence? Acta radiol. 2014;55(4):420-426. doi:10.1177/0284185113500164

6. Goitein O, Di Segni E, Eshet Y, et al. Non-Valvular Findings before Trans-Catheter Aortic Valve Implantation and their Impact on the Procedure. Isr Med Assoc J. 2015;17(12):764-767.

7. Schietinger BJ, Bozlar U, Hagspiel KD, et al. The prevalence of extracardiac findings by multidetector computed tomography before atrial fibrillation ablation. Am Heart J. 2008. doi:10.1016/j.ahj.2007.10.008

8. Sohns C, Sossalla S, Vollmann D, et al. Extra cardiac findings by 64-multidetector computed tomography in patients with symptomatic atrial fibrillation prior to pulmonal vein isolation. Int J Cardiovasc Imaging. 2011. doi:10.1007/s10554-010-9653-9

9. Wissner E, Wellnitz C V., Srivathsan K, et al. Value of multislice computed tomography angiography of the thorax in preparation for catheter ablation for the treatment of atrial fibrillation: The impact of unexpected cardiac and extracardiac findings on patient care. Eur J Radiol. 2009. doi:10.1016/j.ejrad.2008.07.004

10. Ben-Dor I, Waksman R, Hanna NN, et al. Utility of Radiologic Review for Noncardiac Findings on Multislice Computed Tomography in Patients With Severe Aortic Stenosis Evaluated for Transcatheter Aortic Valve Implantation. Am J Cardiol. 2010;105(10):1461-1464. doi:10.1016/j.amjcard.2009.12.071

11. Staab W, Bergau L, Lotz J, et al. Prevalence of noncardiac findings in computed tomography angiography before transcatheter aortic valve replacement. J Cardiovasc Comput Tomogr. 2014;8(3):222-229. doi:10.1016/j.jcct.2014.03.004

12. Showkathali R, Sen A, Brickham B, et al. "Incidental findings" during TAVI work-up: more than just an inconvenience. Eurolntervention. 2015;11(4):465-469. doi:10.4244/eijy14m06_04

13. Lindsay AC, Sriharan M, Lazoura 0 , et al. Clinical and economic consequences of non-cardiac incidental findings detected on cardiovascular computed tomography performed prior to transcatheter aortic valve implantation (TAVI). Int J Cardiovasc Imaging. 2015;31(7):1435-1446. doi:10.1007/s10554-015-0685-z

14. Hussien AF, Jeudy J, Kligerman SJ, et al. Thoracic Incidental Findings in Preoperative Computed Tomography Evaluation for Transcatheter Aortic Valve Implantation (TAVI). J Thorac Imaging. 2016;31(3):183-188. doi:10.1097/RTI.0000000000000208 
15. Shemesh J, Henschke Cl, Shaham D, et al. Ordinal Scoring of Coronary Artery Calcifications on Low-Dose CT Scans of the Chest is Predictive of Death from Cardiovascular Disease. Radiology. 2010;257(2):541-548. doi:10.1148/radiol.10100383

16. MacMahon H, Austin JHM, Gamsu G, et al. Guidelines for Management of Small Pulmonary Nodules Detected on CT Scans: A Statement from the Fleischner Society. Radiology. 2005;237(2):395-400. doi:10.1148/radiol.2372041887

17. Israel GM, Bosniak MA. How I Do It: Evaluating Renal Masses. Radiology. 2005;236:441-450. doi:10.1148/radiol.2362040218

18. Israel GM, Bosniak MA. Follow-up CT of moderately complex cystic lesions of the kidney (Bosniak category IIF). Am J Roentgenol. 2003. doi:10.2214/ajr.181.3.1810627

19. Obuchowski NA, Holden D, Modic MT, et al. Total-Body Screening: Preliminary Results of a Pilot Randomized Controlled Trial. J Am Coll Radiol. 2007;4:604-611. doi:10.1016/j.jacr.2007.04.006

20. Mark DB, Berman DS, Budoff MJ, et al. ACCF/ACR/AHA/NASCI/SAIP/SCAI/SCCT 2010 Expert Consensus Document on Coronary Computed Tomographic Angiography. Circulation. 2010;121(22):2509-2543. doi:10.1161/CIR.0b013e3181d4b618

21. Weijs B, Pisters R, Haest RJ, et al. Patients originally diagnosed with idiopathic atrial fibrillation more often suffer from insidious coronary artery disease compared to healthy sinus rhythm controls. Heart Rhythm. 2012;9(12):1923-1929. doi:10.1016/j.hrthm.2012.08.013

22. Weijs B, de Vos CB, Tieleman RG, et al. The occurrence of cardiovascular disease during 5-year follow-up in patients with idiopathic atrial fibrillation. EP Europace. 2013;15(1):18-23. doi:10.1093/europace/eus203

23. Stachon P, Kaier K, Milde S, et al. Two-year survival of patients screened for transcatheter aortic valve replacement with potentially malignant incidental findings in initial body computed tomography. Eur Heart J Cardiovasc Imaging. 2015. doi:10.1093/ehjci/jev055 
CHAPTER 8

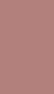

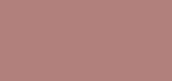


SUMMARY, CONCLUSION AND FUTURE PERSPECTIVES

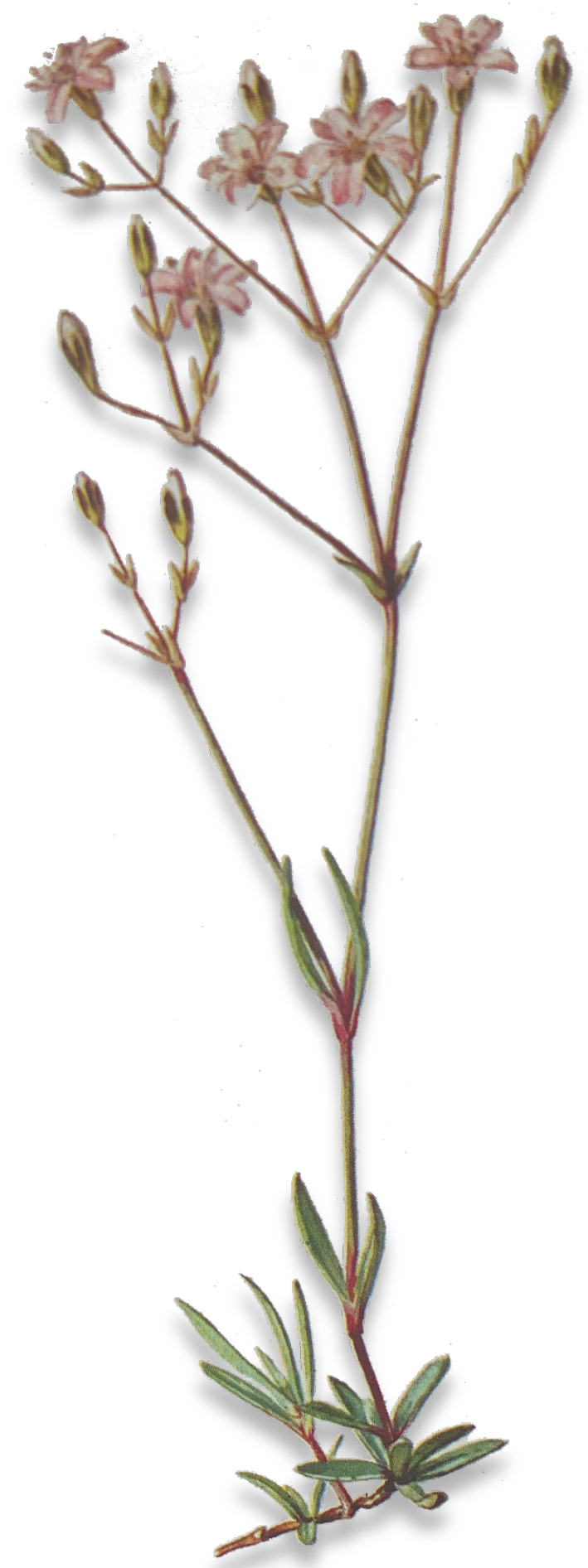




\section{Summary}

Over the past decade, cryoballoon therapy has evolved into a well-recognized and recommended ablation method to perform pulmonary vein isolation (PVI) in the treatment of atrial fibrillation (AF)'. This thesis describes the search for a balance between safety and efficacy in cryoballoon PVI and focusses primarily on the improvement of safety aspects. In the early stage of cryoballoon PVI, the main goal of cryoballoon ablation was to reach similar outcomes compared to the traditional "gold standard" point by point radiofrequency (RF) ablation. The safety aspect was not mentioned regularly in global research. Over the last years, the paradigm has shifted. After the introduction of the second-generation cryoballoon and the proven non-inferiority to RF ablation, more attention was pointed to the safety aspect. During the years in which the research of this dissertation was performed, the number of studies on safety aspects showed a sharp increase.

There are multiple safety aspects to focus on in RF as well as cryoballoon PVI. The most important complications in RF and cryoballoon PVI have been pulmonary vein (PV) stenosis, thrombi and (silent) stroke, tamponade and damage to surrounding tissues. Due to the large diversity in study results, there is no global consensus on precaution measures impeding more rare complications. At this moment the main safety issue in cryoballoon PVI is damage in surrounding tissues due to the dispersion of cryothermal energy. A broad range of safety measures is available to prevent this extra-cardiac damage. Monitoring of critical structures both by compound motor action potentials and measurement of oesophageal temperature (OT) can enhance the safety profile of the procedure. Despite the fact that such precautions are available they are certainly not widely used. Conclusive studies are needed to integrate additional safety measures as part of the standard ablation procedure. Limiting the amount of RF- and cryoenergy while maintaining efficacy is subject of ongoing investigation². (Chapter 2)

\section{Dosing}

With the aim to reduce extra-cardiac damage without affecting treatment efficacy, the dosing of cryoenergy became critically important. Historically, 4-minute applications with a bonus application after isolation was the standard for the firstgeneration cryoballoon ${ }^{3}$. The use of single and shorter applications as well as omitting bonus applications after successful isolation have been explored ${ }^{4-8}$. Protocols with shorter ablation duration have shown encouraging results ${ }^{4,5,9}$. However, this has mainly been demonstrated retrospectively and the optimal cryoballoon duration and dosing strategy are still subject of debate. 
The 123-study was the first to compare standard to new dosing protocols prospectively in a randomized fashion. Results showed that a shortened application duration protocol of medium duration does not reduce acute isolation success compared to the historical standard. Shorter cryoballoon application duration did significantly reduce acute success for the left, but not for the right, PVs. In addition, phrenic nerve injury (PNI) decreased as a result of a reduction of the application duration for the right PVs to less than 2 min. (Chapter 3)

Although long-term success was slightly reduced with shortened application duration, no inferiority was proven. PV analysis showed that reduction was mainly present in the left PVs, although again no inferiority was proven. For the right superior PV, shorter cryoballoon applications (<2 minutes) did not affect PV reconduction while resulting in less PNI. (Chapter 3 and 4)

\section{Monitoring}

Monitoring of critical structures can be used to alarm the operating physician for imminent extra-cardiac damage. It enables the operator to prematurely cease ablation applications to prevent complications. For example, damage to the oesophagus and the vagal nerve could be prevented by monitoring the OT.

OT-based guidance of cryoballoon PVI has shown a significant reduction of thermal oesophageal lesions. Interrupting applications at an OT of $15^{\circ} \mathrm{C}$ has been suggested to prevent oesophageal injury ${ }^{10}$. In Chapter 5 we demonstrated that low OTs emerge on a regular base in clinical practice. A quarter of the patients show OTs below $20^{\circ} \mathrm{C}$, with half of those even below $16^{\circ} \mathrm{C}$. Without oesophageal monitoring this would not be noticed. Since symptoms of oesophageal and vagal damage are usually not linked to a cardiac procedure by the patients, these complications are not always attributed to this procedure and might be under-reported.

Balloon temperature is often used as a surrogate metric for low temperatures in the oesophagus and adjacent structures. However, the results in Chapter 5 showed no clinically significant correlation between minimum balloon temperature and the lowest measured OT. This is in accordance with earlier reports on this subject ${ }^{10,11}$.

\section{Predicting}

Besides dosing and monitoring, imaging is also an important tool to guide PVI procedures. The ability to identify patients at risk enables operators to take precautions for a specific subset of patients. Imaging can be used to make such predictions. For example, the oesophagus to PV (OP) distance as determined on computed tomography (CT) was shown to be such a predictor. A cut-off value for 
an OP distance of $19 \mathrm{~mm}$ showed a $96.2 \%$ sensitivity and a $37.8 \%$ specificity for predicting low OTs. As an OP distance of $<19 \mathrm{~mm}$ for any PV was present in all patients, we recommend routine OT measurement in all patients during cryoballoon therapy to prevent oesophageal related complications. (Chapter 5)

When using imaging as a guiding tool during PVI procedures, it was shown that the use of 3D CT overlay has several benefits over direct fluoroscopy. As the 3D CT overlay image supplies a 3D navigation map, manoeuvring and placing the cryoballoon becomes more straightforward. (Chapter 6) Even though the study population was limited, the study showed that the use of 3D CT overlay has the potential to reduce radiation dose and exposure to contrast dye in cryoballoonbased PVI. Additional advantage of 3D magnetic resonance imaging (MRI) overlay with respect to radiation dose is to be expected.

So pre-procedural CT scans provide detailed anatomical information which can be used before as well as during the procedure. However, incidental findings that identify potential pathologies may be revealed on pre-procedural CT scans. In both the PVI and transcatheter aortic valve replacement population presence of these incidental findings is very common ${ }^{12-23}$. This was confirmed by the results presented in Chapter 7. Furthermore, Chapter 7 contributes to the ongoing debate regarding the beneficial and adverse effects of recognizing incidental findings. Early detection of pathology potentially enables early treatment but simultaneously results in increased costs, increased radiation exposure and possible anxiety for the patient. Based on Chapter 7 anxiety could be reduced by actively informing patients that although the chance of an incidental finding is present, only a small percentage requires therapy. Merely alterations in choices or scheduling could be made due to the gained knowledge. 


\section{Conclusion and future perspectives}

Both efficacy, determined by electrical isolation, and safety, determined by energy dispersion, are a function of numerous factors. Dosing and monitoring strategies are an endeavour to manage these factors. However, the number of interacting factors is such that no single set of numbers or values can be used as a guide for cryoballoon PVI in general.

Unfortunately, all dosing and monitoring parameters available are substitutes for determining the efficacy as well as the safety of the procedure. The main parameter of interest should be transmurality of the ablation energy and the creation of a durable (permanent) lesion. To achieve isolation the energy should reach the epicardium of the atrial wall while to prevent complications the energy should not disperse beyond the epicardium of the heart. Therefore the key focus should be on changing from substitute to direct and real-time measurement of lesion creation. Direct and real-time measurement of the freeze zone would provide the operator with direct feedback on lesion and collateral damage formation. Real-time measurement of this crucial parameter would not only enable a PV or patienttailored approach but even an application tailored approach.

In terms of dosing a first and promising step towards relatively direct measurement was made by adjusting application time to the time to isolation (TTI) of the PV. TTI facilitates addressing every patient and PV uniquely but still does not visualize transmurality itself and it lacks information on the surrounding structures.

The development of the $3^{\text {rd }}$ and $4^{\text {th }}$ generation cryoballoon was mainly focussed on direct measurement of the PV potentials and TTI. Unfortunately, TTI still cannot be measured in every PV. Main reason is that the Achieve catheter is not only used for mapping but also as a guidewire to stabilize the balloon position. This involves advancement of the Achieve catheter past the myocardial sleeves, distal into the $\mathrm{PV}$, thereby obscuring PV potentials. To overcome this problem the design of the Achieve mapping catheter could be improved by creating a wire which can be advanced distally with electrodes that can move independently from the wire (Figure 1). This enables proximal PV potential measurement while stabilizing the balloon distally.

Real-time imaging can be seen as the main opportunity towards an even more direct measurement of transmurality and the extent to which surrounding tissues are reached. Real-time visualisation of ablation related tissue changes using MRI already showed promising results in canine studies ${ }^{24}$. As tissue freezes its MRI 


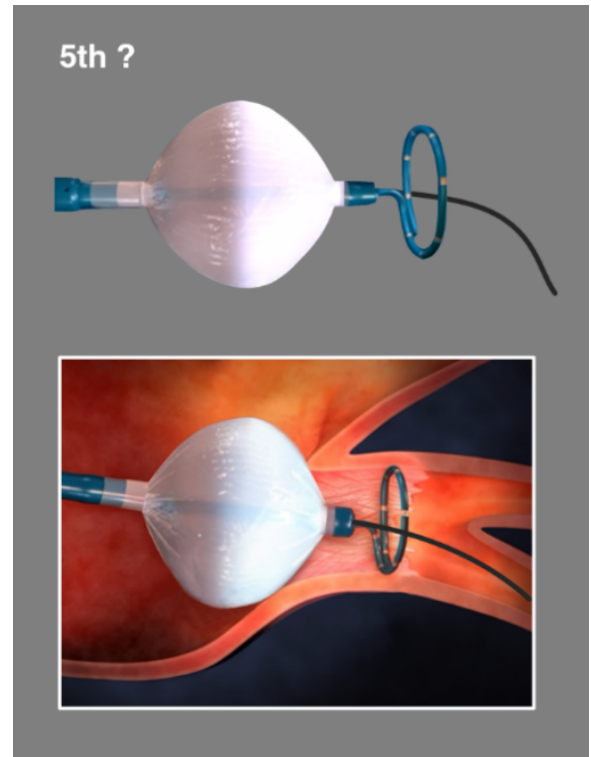

Figure 1 Possible design of the next generation cryoballoon including a wire (black in this figure) which can be advanced distally (bottom panel) with electrodes that can move independently from the wire. This enables proximal PV potential measurement while stabilizing the balloon distally. (Adapted from figures provided by Medtronic Inc.) 
signal diminishes and therefore real-time MRI was shown to successfully visualize the freeze zone, creating a direct measure of effective cooling and ice formation in cardiac and non-cardiac structures.

Historically cryoballoon PVI has seen a fast introduction in clinical practice, merely because of the high efficacy and the straightforward procedure. Optimization efforts in cryoballoon, besides the introduction of the improved balloon, predominantly involved empirical proof by trial and error testing of arbitrarily chosen dosing times.

Therefore it would be of interest to go back to bench-testing now, towards a modelbased assessment of thermodynamic properties of cryoablation. Giaretto recently showed that the efficiency of ablation changes with variations in subtracted heat flux ${ }^{25}$. Despite the very encouraging results of that study, creating a reliable model in bench-testing has shown, underlined by our first-hand experiences, to be challenging (Figure 2-4). Even while using an open-box setting there are still numerous influences complicating the creation of a simplified model and the generation of reliable and reproducible results. Therefore it would be very interesting to see a study on ice-formation and propagation in which tech and health experts would join forces.

Predictors to improve safety have not been studied as thoroughly as markers to improve efficacy. Moreover, the safety aspect has been studied and managed mainly clinically. Knowledge of the biological effect of cryoenergy is lacking while excessive energy delivery is the crucial cause of all main complications in cryoballoon PVI. Therefore a better understanding of thermal behaviour and biophysical properties affecting ice formation and propagation is of key importance in improving the safety aspect. It would enable modelling for differentiated protocol analysis and could predict how to reach the optimal effect of cryoballoon PVI or which biophysical parameters can be used intra-procedurally. 


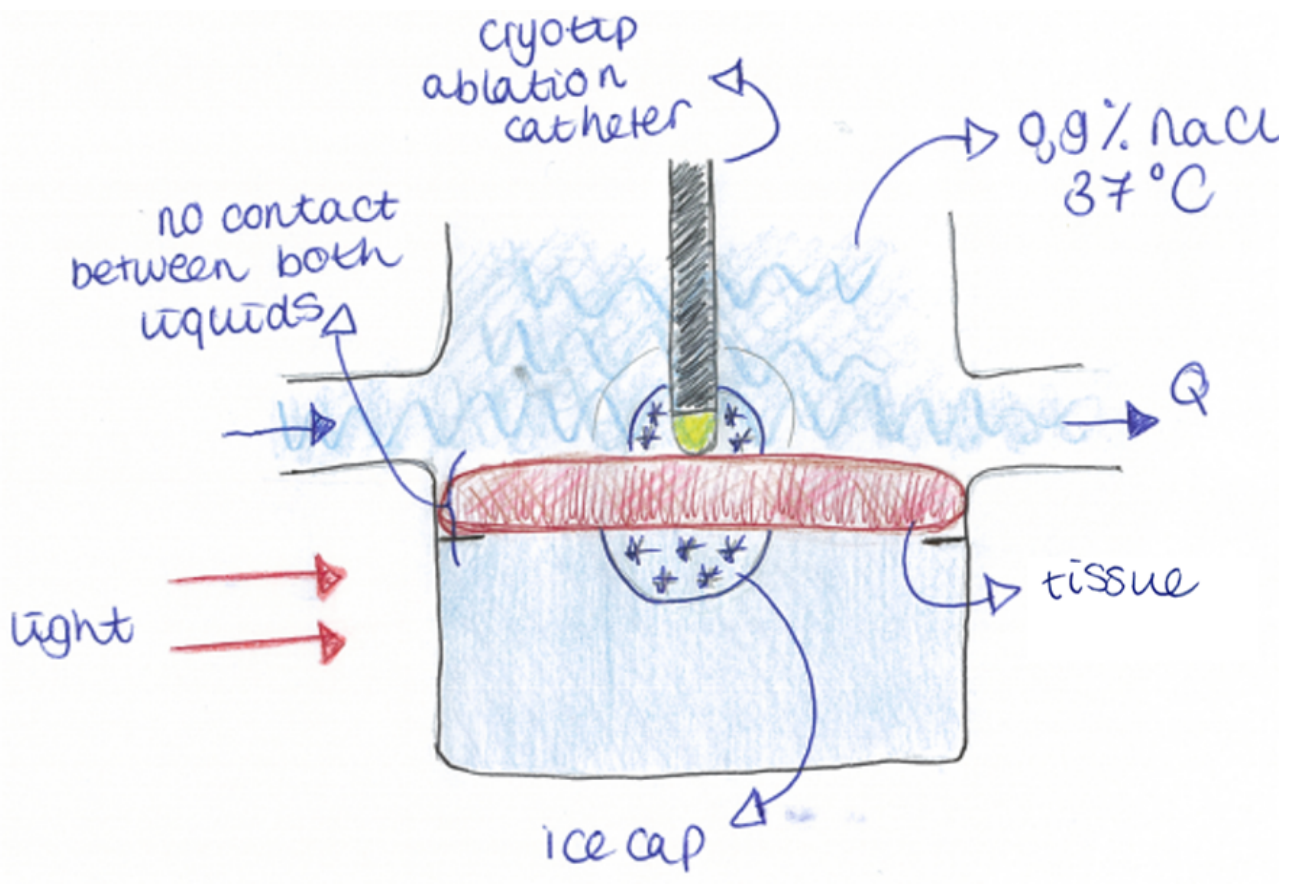

Figure 2 Schematic overview of the model used to perform the bench testing at the University of Twente. The atrium, its wall and its surrounding tissues were modelled as two $0.9 \% \mathrm{NaCl}$ solutions of $37^{\circ} \mathrm{C}$ with a slice of tissue in between. The surrounding of the atrium was represented by a solution without flow, the atrium cavity was represented by the same solution but with flow $\mathrm{Q}$. The tissue was clamped to the walls of the model to ensure there is no contact between the two solutions. Using a cryotip ablation catheter two cycles of ablation were performed. The internal and external icecap created by the ablation in the "atrium" and in the "surrounding" was measured visually using a grid (light intensity, echo and temperature camera measurements failed). 


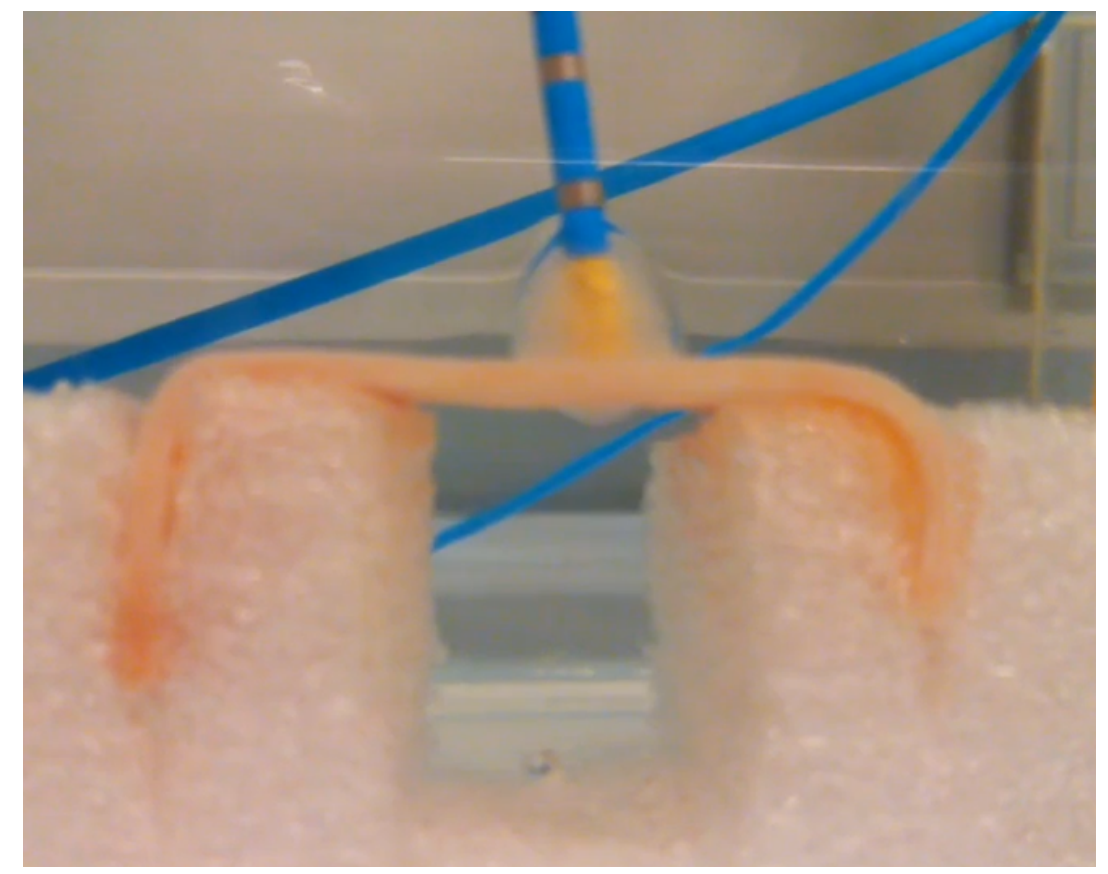

Figure 3 Detailed view of one of the models, without the grid attached. A large "internal" icecap, surrounding the catheter, and a small "external" icecap, at the bottom side of the tissue can be distinguished.

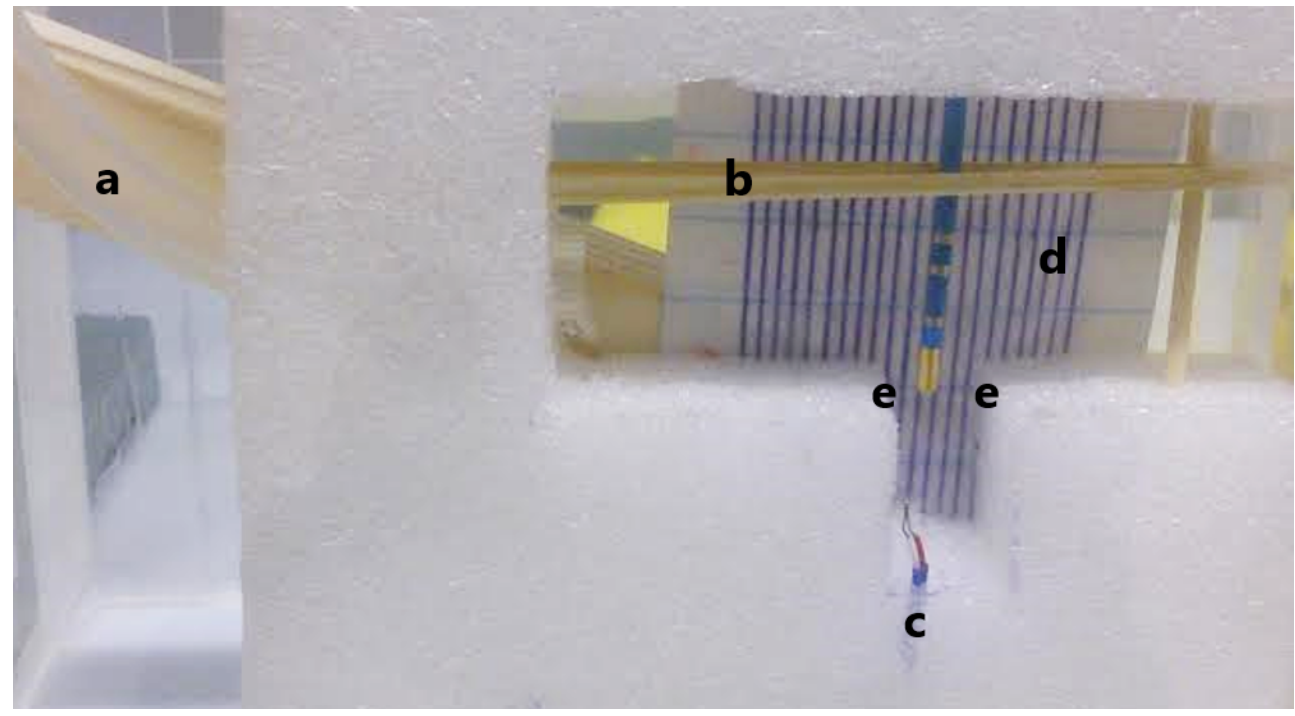

Figure 4 Bench-testing model as used with a. the tube in which a pulsatile flow of $37^{\circ} \mathrm{C}$ ran, b. a wooden frame, to prevent interference with conduction properties, for catheter fixation, $c$. a temperature sensor, in the "cardiac surrounding" area, $d$. the grid for measuring the ice-cap diameters and e. the borders on which the tissue was installed (no tissue in place) 


\section{References}

1. Kirchhof P, Benussi S, Kotecha D, et al. 2016 ESC Guidelines for the management of atrial fibrillation developed in collaboration with EACTS. Eur Heart J. 2016;50(5):e1-e88.

doi:10.1093/ejcts/ezw313

2. Molenaar MMD, Hesselink T, Stevenhagen YJ, et al. Less than 2 min cryoballoon applications result in comparable acute and 6-months success for the treatment of atrial fibrillation but reduced incidence of phrenic nerve palsy: results of the 123-study. Eur Heart J. 2016;37(Abstract supplement):633.

3. Packer DL, Kowal RC, Wheelan KR, et al. Cryoballoon ablation of pulmonary veins for paroxysmal atrial fibrillation: first results of the North American Arctic Front (STOP AF) pivotal trial. J Am Coll Cardiol. 2013;61(16):1713-1723. doi:10.1016/j.jacc.2012.11.064

4. Ciconte G, de Asmundis C, Sieira J, et al. Single 3-minute freeze for second-generation cryoballoon ablation: One-year follow-up after pulmonary vein isolation. Heart Rhythm. 2015;12(4):673-680. doi:10.1016/j.hrthm.2014.12.026

5. Miyazaki S, Hachiya $\mathrm{H}$, Nakamura $\mathrm{H}$, et al. Pulmonary vein isolation using a second-generation cryoballoon in patients with paroxysmal atrial fibrillation: One-year outcome using a single bigballoon 3-minute freeze technique. J Cardiovasc Electrophysiol. 2016;27(12):1375-1380. doi:10.1111/jce.13078

6. Heeger $\mathrm{C}-\mathrm{H}$, Wissner $\mathrm{E}$, Wohlmuth $\mathrm{P}$, et al. Bonus-freeze: benefit or risk? Two-year outcome and procedural comparison of a "bonus-freeze" and "no bonus-freeze” protocol using the secondgeneration cryoballoon for pulmonary vein isolation. Clin Res Cardiol. 2016;105(9):774-782. doi:10.1007/s00392-016-0987-8

7. De Regibus V, lacopino S, Abugattas JP, et al. Single freeze strategy with the second- generation cryballoon for atrial fibrillation: a multicenter international retrospective analysis in a large cohort of patients. J Interv Card Electrophysiol. 2017;49(2):173-180. doi:10.1007/s10840-017-0254-6

8. Tebbenjohanns J, Höfer C, Bergmann L, et al. Shortening of freezing cycles provides equal outcome to standard ablation procedure using second-generation $28 \mathrm{~mm}$ cryoballoon after 15month follow-up. Europace. 2016;18(2):206-210. doi:10.1093/europace/euv189

9. Chun KRJ, Stich M, Fürnkranz A, et al. Individualized cryoballoon energy pulmonary vein isolation guided by real-time pulmonary vein recordings, the randomized ICE-T trial. Heart Rhythm. 2017;14(4):495-500. doi:10.1016/j.hrthm.2016.12.014

10. Fürnkranz A, Bordignon S, Böhmig M, et al. Reduced incidence of esophageal lesions by luminal esophageal temperature-guided second-generation cryoballoon ablation. Heart Rhythm. 2015;12(2):268-274. doi:10.1016/j.hrthm.2014.10.033

11. Fürnkranz A, Bordignon S, Schmidt B, et al. Luminal esophageal temperature predicts esophageal lesions after second-generation cryoballoon pulmonary vein isolation. Heart Rhythm. 2013;10(6):789-793. doi:10.1016/j.hrthm.2013.02.021

12. Martins RP, Muresan L, Sellal J-M, et al. Incidental extracardiac findings in cardiac computed tomography performed before radiofrequency ablation of atrial fibrillation. Pacing Clin Electrophysiol. 2011;34(12):1665-1670. doi:10.1111/j.1540-8159.2011.03194.x

13. Casella M, Perna F, Pontone G, et al. Prevalence and clinical significance of collateral findings detected by chest computed tomography in patients undergoing atrial fibrillation ablation. EP Europace. 2012;14(2):209-216. doi:10.1093/europace/eur300

14. Gufler H, Schulze CG, Wagner S. Incidental findings in computed tomographic angiography for planning percutaneous aortic valve replacement: advanced age, increased cancer prevalence? 
Acta radiol. 2014;55(4):420-426. doi:10.1177/0284185113500164

15. Goitein O, Di Segni E, Eshet Y, et al. Non-Valvular Findings before Trans-Catheter Aortic Valve Implantation and their Impact on the Procedure. Isr Med Assoc J. 2015;17(12):764-767.

16. Schietinger BJ, Bozlar U, Hagspiel KD, et al. The prevalence of extracardiac findings by multidetector computed tomography before atrial fibrillation ablation. Am Heart J. 2008. doi:10.1016/j.ahj.2007.10.008

17. Sohns C, Sossalla S, Vollmann D, et al. Extra cardiac findings by 64-multidetector computed tomography in patients with symptomatic atrial fibrillation prior to pulmonal vein isolation. Int J Cardiovasc Imaging. 2011;27(1):127-134. doi:10.1007/s10554-010-9653-9

18. Wissner E, Wellnitz C V., Srivathsan K, et al. Value of multislice computed tomography angiography of the thorax in preparation for catheter ablation for the treatment of atrial fibrillation: The impact of unexpected cardiac and extracardiac findings on patient care. Eur J Radiol. 2009;72(2):284-288. doi:10.1016/j.ejrad.2008.07.004

19. Ben-Dor I, Waksman R, Hanna NN, et al. Utility of Radiologic Review for Noncardiac Findings on Multislice Computed Tomography in Patients With Severe Aortic Stenosis Evaluated for Transcatheter Aortic Valve Implantation. Am J Cardiol. 2010;105(10):1461-1464. doi:10.1016/j.amjcard.2009.12.071

20. Staab W, Bergau L, Lotz J, et al. Prevalence of noncardiac findings in computed tomography angiography before transcatheter aortic valve replacement. J Cardiovasc Comput Tomogr. 2014;8(3):222-229. doi:10.1016/j.jcct.2014.03.004

21. Showkathali R, Sen A, Brickham B, et al. "Incidental findings" during TAVI work-up: more than just an inconvenience. Eurolntervention. 2015;11(4):465-469. doi:10.4244/EIJY14M06_04

22. Lindsay AC, Sriharan M, Lazoura 0 , et al. Clinical and economic consequences of non-cardiac incidental findings detected on cardiovascular computed tomography performed prior to transcatheter aortic valve implantation (TAVI). Int J Cardiovasc Imaging. 2015;31(7):1435-1446. doi:10.1007/s10554-015-0685-z

23. Hussien AF, Jeudy J, Kligerman SJ, et al. Thoracic Incidental Findings in Preoperative Computed Tomography Evaluation for Transcatheter Aortic Valve Implantation (TAVI). J Thorac Imaging. 2016;31(3):183-188. doi:10.1097/RTI.0000000000000208

24. Lichter J, Kholmovski EG, Coulombe N, et al. Real-time magnetic resonance imaging-guided cryoablation of the pulmonary veins with acute freeze-zone and chronic lesion assessment. EP Europace. 2019;21(1):154-162. doi:10.1093/europace/euy089

25. Giaretto V, Ballatore A, Passerone C, et al. Thermodynamic properties of atrial fibrillation cryoablation: a model-based approach to improve knowledge on energy delivery. J R Soc Interface. 2019;16(158):20190318. doi:10.1098/rsif.2019.0318 
CHAPTER 9 
NEDERLANDSE SAMENVATTING,

CONCLUSIE EN TOEKOMSTPERSPECTIEVEN

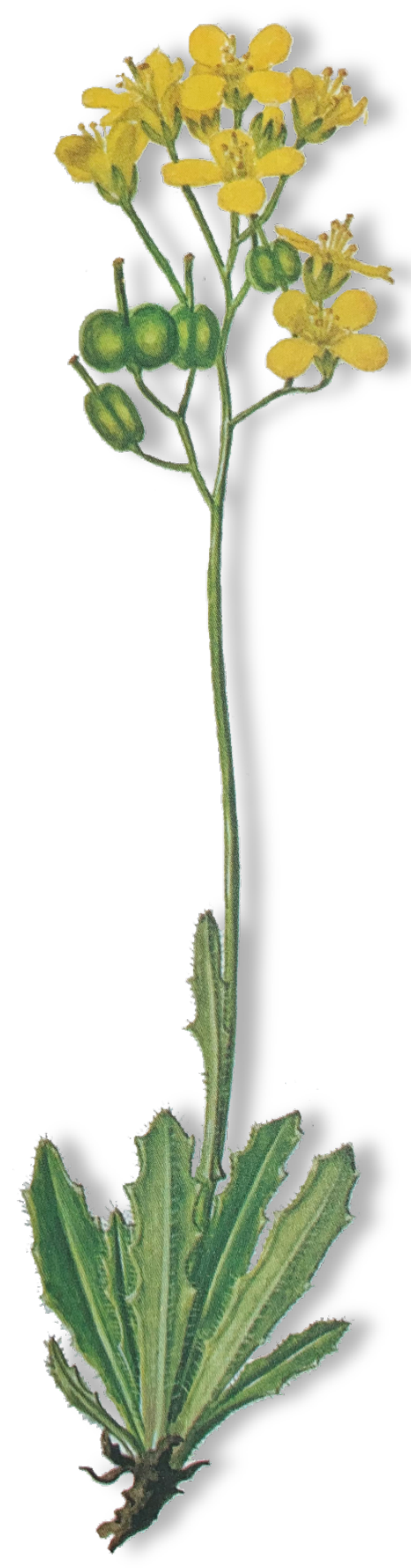




\section{Nederlandse samenvatting}

Ablatie door middel van cryoballon therapie heeft zich in het afgelopen decennia ontwikkeld tot een gevestigde en aanbevolen methode voor het uitvoeren van pulmonaal venen isolatie (PVI) in het kader van de behandeling van atriumfibrilleren (AF)'. In dit proefschrift wordt de zoektocht naar een balans tussen veiligheid en effectiviteit van cryoballon PVI beschreven en wordt primair gefocust op de mogelijke verbeteringen van het veiligheidsaspect.

In eerste instantie zijn ontwikkelingen en studies met betrekking tot cryoballon PVI voornamelijk gericht geweest op de effectiviteit. Er werd in eerste instantie veel aandacht gericht op het behalen van resultaten die vergelijkbaar waren met de klassieke "gouden standaard" radiofrequente (RF) ablatie. Toentertijd was het veiligheidsaspect een niet vaak genoemd onderwerp in wereldwijd onderzoek. De afgelopen jaren is de focus verlegd. Na de introductie van de tweede generatie cryoballon en nadat de non-inferioriteit ten opzicht van RF ablatie bewezen werd kwam het veiligheidsaspect meer voor het voetlicht. Gedurende de jaren waarin het onderzoek uit dit proefschrift uitgevoerd is, was er een flinke stijging waar te nemen in het aantal studies dat het veiligheidsaspect behandelde.

In zowel RF als cryoballon PVI zijn er verscheidene veiligheidsaspecten om rekening mee te houden. De belangrijkste complicaties van beide methoden waren historisch gezien pulmonaal venen (PV) stenosis, thrombusvorming en (stille) cerebrovasculaire accidenten, tamponade en schade aan omliggende weefsels. Om schade aan omliggende weefsels te voorkomen is er een verscheidenheid aan veiligheidsmaatregelen beschikbaar. Het veiligheidsprofiel van de procedure kan verbeterd worden door belangrijke structuren te monitoren, bijvoorbeeld door het meten van compound motor actie potentialen en oesophagus temperatuur. Het feit dat deze voorzorgsmaatregelen beschikbaar zijn betekent echter niet dat ze alom gebruikt worden. $\mathrm{Er}$ is behoefte aan conclusieve studies om deze extra voorzorgsmaatregelen als standaard te integreren in reguliere ablatie procedures. Het beperken van de toegediende RF of cryo energie met behoud van de effectiviteit is een focus van momenteel lopend onderzoek². (Hoofdstuk 2)

\section{Dosering}

Het doseren van cryo energie is een belangrijk focus geworden in pogingen om extra-cardiale schade te verminderen zonder de effectiviteit van de behandeling te beïnvloeden. Historisch gezien zijn meerdere applicaties van 4 minuten, inclusief bonus applicatie na isolatie, de standaarddosering geweest voor cryoballon PVI 
met de eerste generatie cryoballon ${ }^{3}$. Het gebruik van een enkele alsook kortere applicaties is onderzocht, net als het afzien van bonus applicaties na succesvolle isolatie ${ }^{4-8}$. Protocollen waarin kortere applicatietijden gebruikt worden hebben veelbelovende resultaten laten zien ${ }^{4,5,9}$. Dit waren echter voornamelijk retrospectieve studies en zodoende zijn de optimale cryoballon applicatietijd en dosering strategie nog steeds onderwerp van discussie.

De 123-studie was de eerste gerandomiseerde studie waarin een vergelijking tussen standaard en nieuwe dosering strategieën gemaakt werd. Deze studie liet zien dat er geen (acuut) verschil was wanneer de applicatieduur verkort werd ten opzichte van de historische standaarden, tot applicatie tijden van gemiddelde duur. Kortere applicatietijden hadden een significant negatief effect op het acute succes van de linker maar niet van de rechter PVs. Bovendien resulteerde de verkorting van de applicatieduur tot korter dan twee minuten in een vermindering van nervus phrenicus letsel. (Hoofdstuk 3)

Hoewel er een licht negatief effect op het lange termijn succes waar te nemen was, was er geen bewijs voor inferioriteit voor deze kortere applicatieduur. Voor de rechter bovenste PV geldt dat kortere cryoballon applicaties ( $<2$ minuten) geen effect hadden op de reconductie terwijl er minder nervus phrenicus letsel optrad. (Hoofdstuk 3 en 4)

\section{Monitoring}

Om de operateur te alarmeren bij dreigende extra-cardiale schade kan er gebruik gemaakt worden van monitoring van kwetsbare weefsels. Op deze manier wordt de operateur in staat gesteld om tijdig de applicatie te onderbreken en op die manier complicaties te voorkomen. Het monitoren van de oesophagus temperatuur kan bijvoorbeeld gebruikt worden om schade aan de oesophagus en de nervus vagus te voorkomen.

Het gebruik van oesophagus temperatuur monitoring tijdens cryoballon PVI zorgt voor een significante vermindering in temperatuur gerelateerde oesophagus laesies. Om oesophagus letsel te voorkomen wordt aangeraden om applicaties te onderbreken bij een temperatuur van $15^{\circ} \mathrm{C}^{10}$. In Hoofdstuk 5 laten we zien dat lage oesophagus temperaturen zeer regelmatig voorkomen in de reguliere klinische praktijk. In een kwart van de patiënten werden oesophagus temperaturen onder de $20^{\circ} \mathrm{C}$ gemeten, in de helft daarvan daalde de temperatuur zelfs tot onder de $16^{\circ} \mathrm{C}$. Zonder oesophagus temperatuur monitoring zouden deze lage temperaturen onopgemerkt zijn gebleven. Oesophagus schade en schade aan de nervus vagus 
zorgen voor symptomen die door patiënten niet altijd aan cardiale procedures gerelateerd worden. Zodoende is het zeer wel mogelijk dat deze schade onder gerapporteerd blijft.

Als surrogaat voor het meten van de oesophagus temperatuur wordt er vaak gebruik gemaakt van de ballon temperatuur. De resultaten in Hoofdstuk 5 laten echter zien dat er geen klinisch significante correlatie te vinden is tussen de minimale ballon temperatuur en de laagst gemeten oesophagus temperatuur. Dit is conform eerdere studies naar dit onderwerp ${ }^{10,11}$.

\section{Voorspellers}

Naast doseren en monitoren is beeldvorming ook een belangrijk instrument om PVI procedures te ondersteunen. Beeldvorming kan gebruikt worden voor het aanmerken van patiënten die tot een risicogroep behoren. Door dit voorafgaand aan de procedure te doen kunnen er in deze groep extra voorzorgsmaatregelen getroffen worden. De afstand van oesophagus tot PV op computed tomography (CT) beelden is een dergelijke voorspeller van verhoogd risico. Een afkapwaarde van $19 \mathrm{~mm}$ kan gebruikt worden om lage oesophagus temperaturen te voorspellen met een sensitiviteit van $96.2 \%$ en een specificiteit van $37.8 \%$. In elke patiënt was er minimaal 1 PV met een oesophagus tot PV afstand van $<19 \mathrm{~mm}$. Daarom wordt aangeraden om, om oesophagus gerelateerde complicaties te voorkomen, monitoring van oesophagus temperatuur reguliere klinische praktijk te maken. (Hoofdstuk 5)

In het kader van ondersteuning van PVI procedures middels beeldvormende technieken heeft 3D CT een aantal voordelen ten opzichte van directe röntgen doorlichting. Doordat de 3D CT een 3D beeld voor navigatie creëert, wordt het manoeuvreren en plaatsen van de cryoballon een stuk gemakkelijker. (Hoofdstuk 6) Ondanks de kleine populatie toonde onze studie aan dat er potentieel minder stralingsdosis en blootstelling aan contrastvloeistof gedurende de cryoballon PVI procedure is wanneer er gebruik wordt gemaakt van 3D CT. Verdere winst op het gebied van stralingsdosis is te verwachten wanneer in plaats van 3D CT gebruik gemaakt zou worden van 3D magnetic resonance imaging (MRI).

Pre-procedurele CT scans geven gedetailleerde anatomische informatie welke gebruikt kan worden zowel voorafgaand aan als tijdens de procedure. Deze preprocedurele CT scans kunnen echter ook "bijvangst" met zicht meebrengen in de vorm van toevalsbevindingen van potentiele pathologieën. In zowel de PVI als de percutane aortaklepvervanging populatie komen deze toevalsbevindingen 
veelvuldig voor ${ }^{12-23}$. De resultaten in Hoofdstuk 7 onderschrijven deze eerdere observaties. Verder draagt hoofdstuk 7 bij aan de huidige discussie omtrent de voordelige en nadelige effecten van het doen van toevalsbevindingen. Een vroege opsporing van extracardiale afwijkingen kan potentieel een snelle en vroege behandeling mogelijk maken. Tegelijkertijd resulteren toevalsbevindingen in extra zorgkosten en daarnaast voor de patiënt ook in hogere stralingsbelasting en mogelijk in angst. Op basis van de resultaten van hoofdstuk 7 kan er bij de voorlichting van patiënten vermeld worden dat, ondanks dat de kans op een toevalsbevinding aanwezig is, er slechts een klein percentage is waarbij ook daadwerkelijk een behandeling noodzakelijk is. Hiermee kan mogelijk de angst bij patiënten verminderd worden. 


\section{Conclusie en toekomstperspectieven}

Zowel effectiviteit, bepaald door elektrische isolatie, als veiligheid, bepaald door energie dispersie, zijn een resultante van verscheidene factoren. Met behulp van dosering en monitoring strategieën wordt er gepoogd deze factoren onder controle te krijgen. De factoren die hierin met elkaar interacteren zijn echter zo talrijk dat er niet één unieke set van waarden gebruikt kan worden als leidraad voor cryoballon PVI in het algemeen.

Alle dosering en monitoring parameters die op dit moment beschikbaar zijn, zijn helaas nog steeds surrogaat parameters voor het bepalen van de effectiviteit en veiligheid van de procedure. De transmuraliteit van de ablatie energie en het creëren van duurzame (permanente) laesies zijn hierin namelijk de belangrijkste factoren. Om isolatie te bereiken moet de ablatie energie door de atriumwand heen de buitenwand van het atrium (epicard) bereiken en om complicaties te voorkomen moet voorkomen worden dat de energie zich verder verspreidt tot buiten het hart. Daarom zou de grootste focus momenteel moeten liggen op het overstappen van surrogaat parameters naar directe en real-time registratie van laesie vorming. Door middel van directe en real-time metingen zou de operateur directe feedback kunnen krijgen over (cryo) laesie vorming en het optreden van eventuele schade aan omliggende weefsels. Real-time registratie van deze cruciale parameter zou niet alleen een PV of een patiënt specifieke aanpak maar zelfs een applicatie specifieke aanpak mogelijk maken. Met betrekking tot dosering is een eerste veelbelovende stap in de richting van relatief directe registratie gezet door het aanpassen van de applicatietijd aan de tijd tot isolatie (TTI) van de PV. Met behulp van TTI kan patiënt- en PV-specifiek geregistreerd worden. Het brengt echter nog steeds de transmuraliteit zelf niet in beeld en het geeft geen informatie over de omliggende weefsels.

De ontwikkeling van de derde en vierde generatie cryoballonnen was voornamelijk op directe registratie van PV potentialen en TTI gericht. Helaas kan de TTI nog steeds niet in elke PV gemeten worden. De voornaamste reden hiervoor is dat de Achieve katheter niet alleen gebruikt wordt voor mapping maar ook als voerdraad om de ballon in de gewenste positie te stabiliseren. Onderdeel hiervan is dat de Achieve katheter opgevoerd wordt in de PV waardoor de PV potentialen niet meer te registreren zijn. Het design van de Achieve katheter zou aangepast kunnen worden om dit probleem op te lossen. Door de introductie van een voerdraad die zich onafhankelijk van de elektrodes kan bewegen kunnen PV potentialen proximaal gemeten worden terwijl de voerdraad distaal voor stabilisatie zorgt (Figuur 1). 


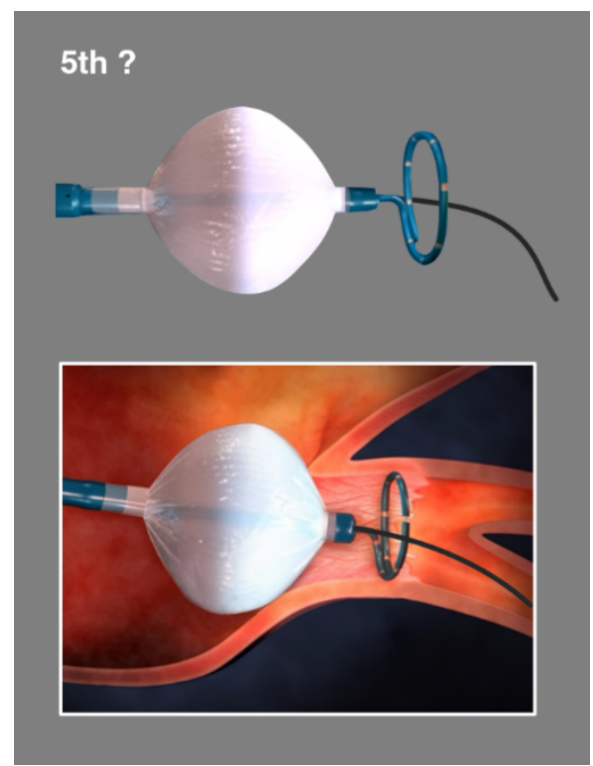

Figure 1 Mogelijk design van de volgende generatie cryoballon. Door de introductie van een voerdraad (zwart in dit figuur) die zich onafhankelijk van de elektrodes kan bewegen kunnen PV potentialen proximaal gemeten worden terwijl de voerdraad distaal voor stabilisatie zorgt (onderste vak). (Figuur op basis van door Medtronic Inc. verstrekte figuren) 
De voornaamste kansen voor directere registratie van transmuraliteit en de mate waarin omliggende weefsels bereikt worden liggen bij real-time beeldvorming. Real-time visualisatie van verandering in weefsel gedurende ablatie middels MRI heeft al veelbelovende resultaten laten zien in studies met honden ${ }^{24}$. Wanneer weefsel bevriest verliest het zijn MRI signaal. Op deze manier kon de vries zone, wat een directe meting is van effectieve bevriezing en ijsformatie in zowel cardiale als niet cardiale structuren, succesvol real-time in beeld gebracht.

De cryoballon heeft een snelle introductie in de klinische praktijk gekend, voornamelijk vanwege de hoge effectiviteit en de laagdrempeligheid van het uitvoeren van de procedure. Pogingen om de procedure te optimaliseren hebben zich, op de introductie van de nieuwe ballonnen na, voornamelijk gericht op het verkrijgen van empirisch bewijs. Dit is gedaan door het trial-and-error principe toe te passen op arbitrair gekozen doseringen qua applicatie duur. Zodoende zou het interessant zijn om een stap terug te doen naar de basis en meer onderzoek te doen naar de thermodynamische eigenschappen van cryoablatie. Recent is aangetoond dat de effectiviteit van ablatie afhankelijk is van variaties in de onttrokken koude flux ${ }^{25}$. Ondanks de veelbelovende resultaten van deze studie is het uitdagend gebleken, ook in onze eigen pogingen, om een betrouwbaar model in lab opstelling te creëren (Figuur 2-4). Zelfs wanneer er gebruik wordt gemaakt van een open doos opstelling zijn er nog steeds vele factoren van invloed. Hierdoor is het creëren van een model en het genereren van reproduceerbare resultaten erg uitdagend. Het zou hoogst interessant zijn om een studie uit te voeren naar ijsformatie en koude propagatie waarin technische en klinische experts de handen ineenslaan.

Samenvattend zijn voorspellers waarmee veiligheid vergroot kan worden zijn nog niet zo uitgebreid bestudeerd als voorspellers voor hogere effectiviteit. Bovendien is het veiligheidsaspect voornamelijk in klinische setting bestudeerd. Er is weinig tot niets bekend over het biologische effect van cryo energie terwijl overmatige energieafgifte dè cruciale reden is van de voornaamste complicaties van cryoballon PVI. Beter begrip van thermisch gedrag en biofysische parameters die de ijsformatie beïnvloeden is de sleutel naar verbeteringen in het veiligheidsaspect van cryoballon PVI. Het zou ons in staat stellen om modellen te creëren waarmee gedifferentieerde protocolanalyses uitgevoerd kunnen worden. Hiermee zou voorspeld kunnen worden hoe we het optimale effect van cryoballon PVI kunnen bereiken en welke biofysische parameters we gedurende de procedure kunnen gebruiken. 


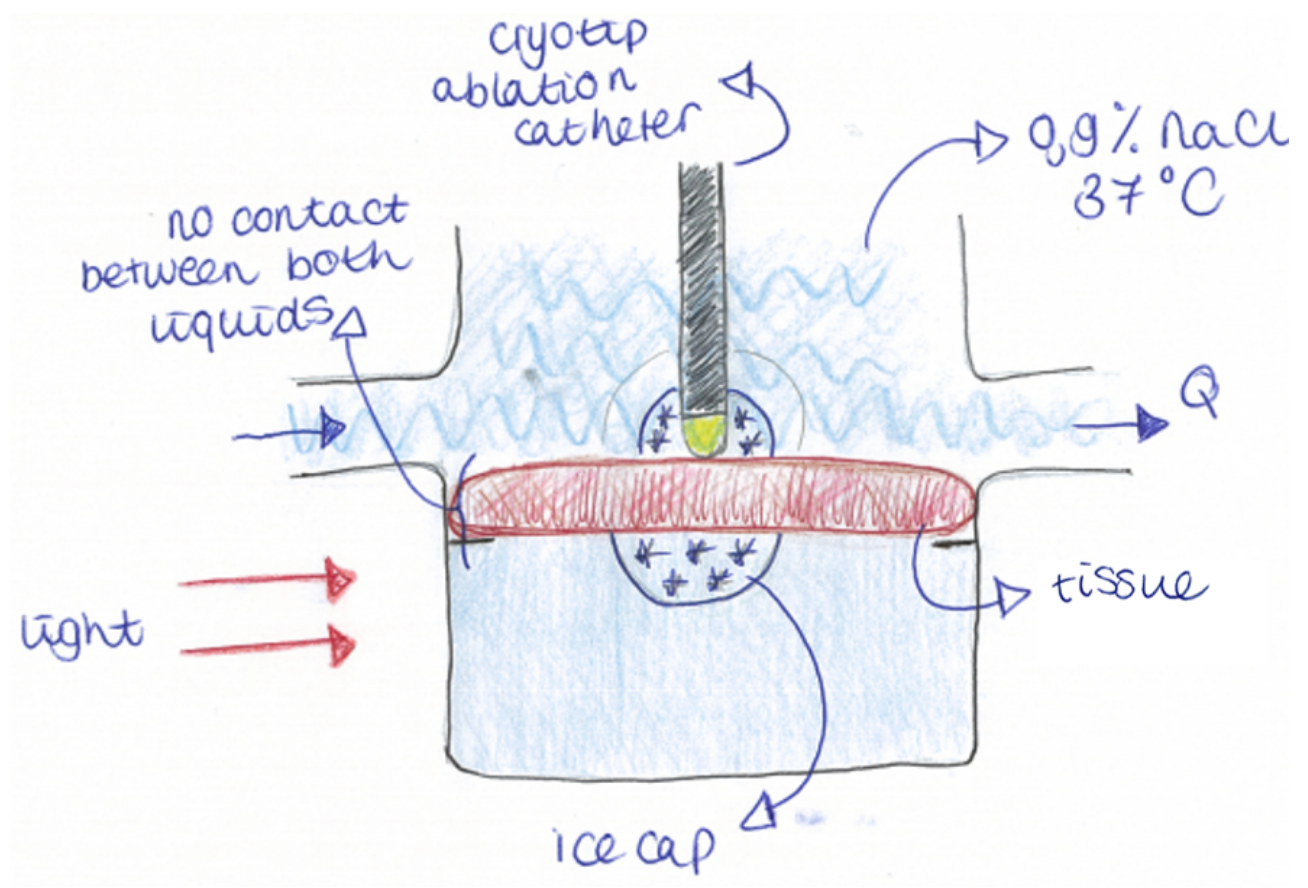

Figure 2 Schematische weergave van het model wat gebruikt is voor de lab opstelling op de Universiteit Twente. Het atrium, de atriumwand en de omliggende weefsels zijn gemodelleerd middels twee $0.9 \% \mathrm{NaCl}$ oplossingen van $37^{\circ} \mathrm{C}$ met een plakje weefsel er tussenin. De omgeving van het atrium werd gerepresenteerd door een vloeistof zonder flow, de atriumholte werd gerepresenteerd door dezelfde vloeistof mèt flow Q. Het weefsel werd tegen de wand van het model geklemd om ervoor te zorgen dat er geen contact was tussen de twee vloeistoffen. Met een cryotip ablatiecatheter werden er twee cycli van ablatie uitgevoerd. De interne en externe ijskap die door de ablatie ontstonden in het gemodelleerde "atrium" en in de "omgeving" werd visueel gemeten met behulp van een raster (nadat licht intensiteit, echo en temperatuur camera metingen niet geschikt bleken). 


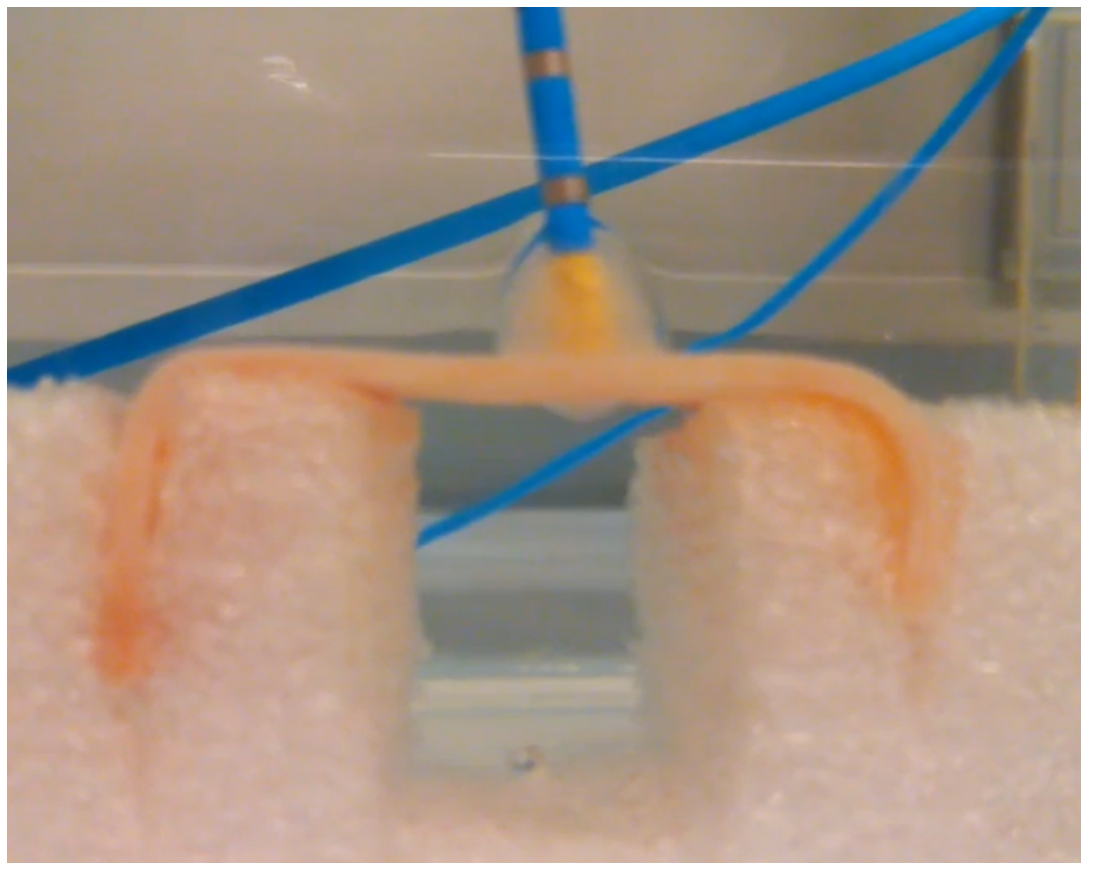

Figure 3 Gedetailleerde weergave van een van de modellen, hierin is het raster nog niet geplaatst. Om de katheter heen is een grote “interne” ijskap te onderscheiden, net als een kleinere "externe" ijskap aan de onderkant van het weefsel.

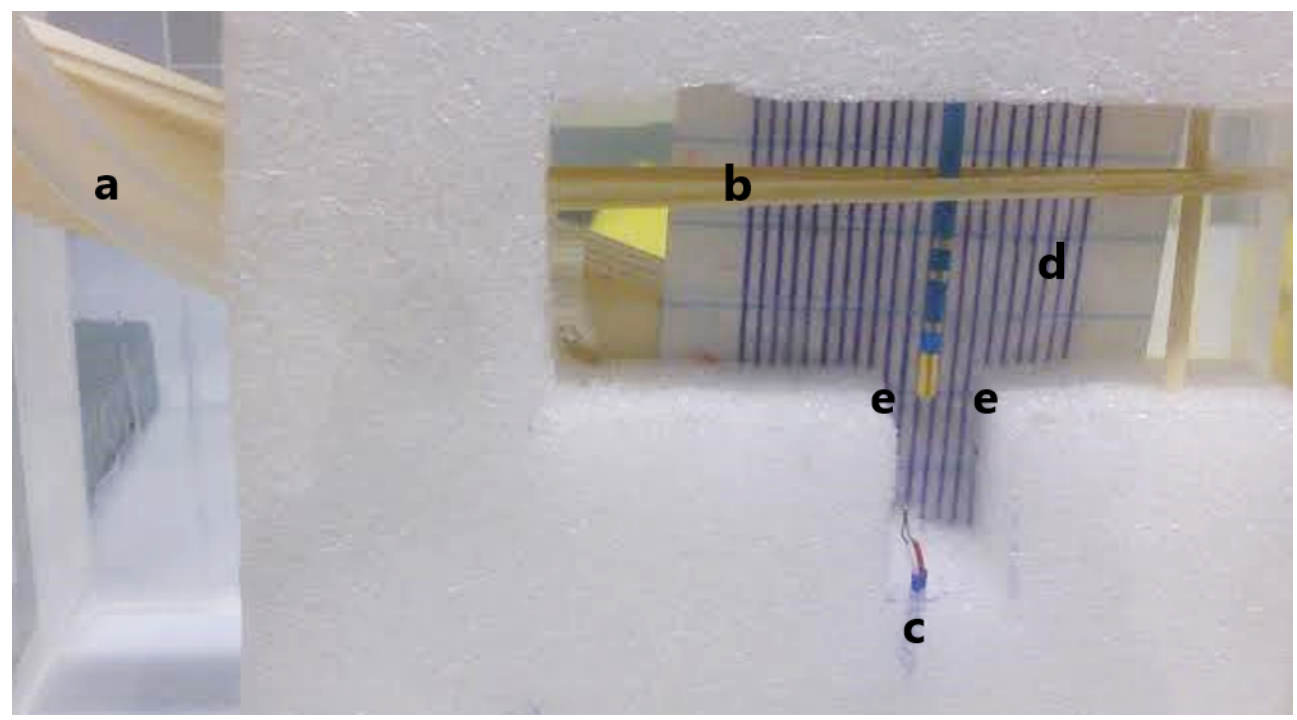

Figure 4 Lab opstelling zoals gebruikt met a. de buis waardoor de pulsatiele flow van $37^{\circ} \mathrm{C}$ stroomde, b. een houten frame, om interferentie met geleidingseigenschappen te voorkomen, om de katheter in te fixeren, c. een temperatuur sensor, die zich in de "omgeving" van het hart bevindt, $d$. het raster waarop de afmeting van de ijskap diameters gemeten werd en e. de wanden waartegen het weefsel werd geklemd (geen weefsel aanwezig in dit figuur). 


\section{Referenties}

1. Kirchhof P, Benussi S, Kotecha D, et al. 2016 ESC Guidelines for the management of atrial fibrillation developed in collaboration with EACTS. Eur Heart J. 2016;50(5):e1-e88.

doi:10.1093/ejcts/ezw313

2. Molenaar MMD, Hesselink T, Stevenhagen YJ, et al. Less than 2 min cryoballoon applications result in comparable acute and 6-months success for the treatment of atrial fibrillation but reduced incidence of phrenic nerve palsy: results of the 123-study. Eur Heart J. 2016;37(Abstract supplement):633.

3. Packer DL, Kowal RC, Wheelan KR, et al. Cryoballoon ablation of pulmonary veins for paroxysmal atrial fibrillation: first results of the North American Arctic Front (STOP AF) pivotal trial. J Am Coll Cardiol. 2013;61(16):1713-1723. doi:10.1016/j.jacc.2012.11.064

4. Ciconte G, de Asmundis C, Sieira J, et al. Single 3-minute freeze for second-generation cryoballoon ablation: One-year follow-up after pulmonary vein isolation. Heart Rhythm. 2015;12(4):673-680. doi:10.1016/j.hrthm.2014.12.026

5. Miyazaki $\mathrm{S}$, Hachiya $\mathrm{H}$, Nakamura $\mathrm{H}$, et al. Pulmonary vein isolation using a second-generation cryoballoon in patients with paroxysmal atrial fibrillation: One-year outcome using a single bigballoon 3-minute freeze technique. J Cardiovasc Electrophysiol. 2016;27(12):1375-1380. doi:10.1111/jce.13078

6. Heeger C-H, Wissner E, Wohlmuth P, et al. Bonus-freeze: benefit or risk? Two-year outcome and procedural comparison of a "bonus-freeze" and "no bonus-freeze" protocol using the secondgeneration cryoballoon for pulmonary vein isolation. Clin Res Cardiol. 2016;105(9):774-782. doi:10.1007/s00392-016-0987-8

7. De Regibus V, lacopino S, Abugattas JP, et al. Single freeze strategy with the second- generation cryballoon for atrial fibrillation: a multicenter international retrospective analysis in a large cohort of patients. J Interv Card Electrophysiol. 2017;49(2):173-180. doi:10.1007/s10840-017-0254-6

8. Tebbenjohanns J, Höfer C, Bergmann L, et al. Shortening of freezing cycles provides equal outcome to standard ablation procedure using second-generation $28 \mathrm{~mm}$ cryoballoon after 15month follow-up. Europace. 2016;18(2):206-210. doi:10.1093/europace/euv189

9. Chun KRJ, Stich M, Fürnkranz A, et al. Individualized cryoballoon energy pulmonary vein isolation guided by real-time pulmonary vein recordings, the randomized ICE-T trial. Heart Rhythm. 2017;14(4):495-500. doi:10.1016/j.hrthm.2016.12.014

10. Fürnkranz A, Bordignon S, Böhmig M, et al. Reduced incidence of esophageal lesions by luminal esophageal temperature-guided second-generation cryoballoon ablation. Heart Rhythm. 2015;12(2):268-274. doi:10.1016/j.hrthm.2014.10.033

11. Fürnkranz A, Bordignon S, Schmidt B, et al. Luminal esophageal temperature predicts esophageal lesions after second-generation cryoballoon pulmonary vein isolation. Heart Rhythm. 2013;10(6):789-793. doi:10.1016/j.hrthm.2013.02.021

12. Martins RP, Muresan L, Sellal J-M, et al. Incidental extracardiac findings in cardiac computed tomography performed before radiofrequency ablation of atrial fibrillation. Pacing Clin Electrophysiol. 2011;34(12):1665-1670. doi:10.1111/j.1540-8159.2011.03194.x

13. Casella M, Perna F, Pontone $G$, et al. Prevalence and clinical significance of collateral findings detected by chest computed tomography in patients undergoing atrial fibrillation ablation. EP Europace. 2012;14(2):209-216. doi:10.1093/europace/eur300

14. Gufler H, Schulze CG, Wagner S. Incidental findings in computed tomographic angiography for planning percutaneous aortic valve replacement: advanced age, increased cancer prevalence? 
Acta radiol. 2014;55(4):420-426. doi:10.1177/0284185113500164

15. Goitein O, Di Segni E, Eshet Y, et al. Non-Valvular Findings before Trans-Catheter Aortic Valve Implantation and their Impact on the Procedure. Isr Med Assoc J. 2015;17(12):764-767.

16. Schietinger BJ, Bozlar U, Hagspiel KD, et al. The prevalence of extracardiac findings by multidetector computed tomography before atrial fibrillation ablation. Am Heart J. 2008. doi:10.1016/j.ahj.2007.10.008

17. Sohns C, Sossalla S, Vollmann D, et al. Extra cardiac findings by 64-multidetector computed tomography in patients with symptomatic atrial fibrillation prior to pulmonal vein isolation. Int J Cardiovasc Imaging. 2011;27(1):127-134. doi:10.1007/s10554-010-9653-9

18. Wissner E, Wellnitz C V., Srivathsan K, et al. Value of multislice computed tomography angiography of the thorax in preparation for catheter ablation for the treatment of atrial fibrillation: The impact of unexpected cardiac and extracardiac findings on patient care. Eur J Radiol. 2009;72(2):284-288. doi:10.1016/j.ejrad.2008.07.004

19. Ben-Dor I, Waksman R, Hanna NN, et al. Utility of Radiologic Review for Noncardiac Findings on Multislice Computed Tomography in Patients With Severe Aortic Stenosis Evaluated for Transcatheter Aortic Valve Implantation. Am J Cardiol. 2010;105(10):1461-1464. doi:10.1016/j.amjcard.2009.12.071

20. Staab W, Bergau L, Lotz J, et al. Prevalence of noncardiac findings in computed tomography angiography before transcatheter aortic valve replacement. J Cardiovasc Comput Tomogr. 2014;8(3):222-229. doi:10.1016/j.jcct.2014.03.004

21. Showkathali R, Sen A, Brickham B, et al. "Incidental findings" during TAVI work-up: more than just an inconvenience. Eurolntervention. 2015;11(4):465-469. doi:10.4244/EIJY14M06_04

22. Lindsay AC, Sriharan M, Lazoura 0 , et al. Clinical and economic consequences of non-cardiac incidental findings detected on cardiovascular computed tomography performed prior to transcatheter aortic valve implantation (TAVI). Int J Cardiovasc Imaging. 2015;31(7):1435-1446. doi:10.1007/s10554-015-0685-z

23. Hussien AF, Jeudy J, Kligerman SJ, et al. Thoracic Incidental Findings in Preoperative Computed Tomography Evaluation for Transcatheter Aortic Valve Implantation (TAVI). J Thorac Imaging. 2016;31(3):183-188. doi:10.1097/RTI.0000000000000208

24. Lichter J, Kholmovski EG, Coulombe N, et al. Real-time magnetic resonance imaging-guided cryoablation of the pulmonary veins with acute freeze-zone and chronic lesion assessment. EP Europace. 2019;21(1):154-162. doi:10.1093/europace/euy089

25. Giaretto V, Ballatore A, Passerone C, et al. Thermodynamic properties of atrial fibrillation cryoablation: a model-based approach to improve knowledge on energy delivery. J R Soc Interface. 2019;16(158):20190318. doi:10.1098/rsif.2019.0318 
CHAPTER 10 
PORTFOLIO

DANKWOORD

CURRICULUM VITAE

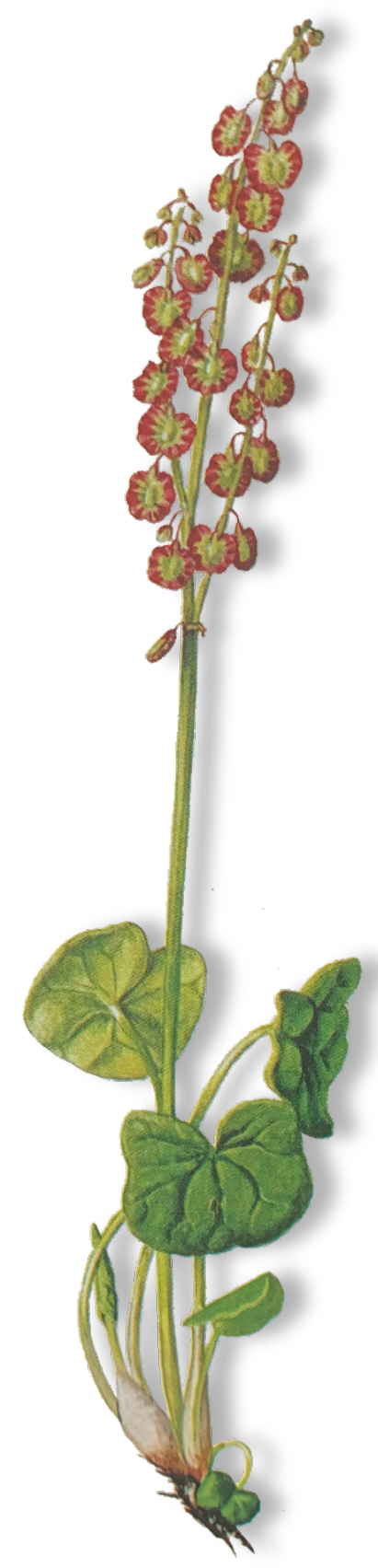




\section{Portfolio}

\section{Publicaties}

Molenaar MMD, Oude Velthuis B, Scholten MF, Stevenhagen JY, Wesselink WA, Van Opstal JM. Optimization of cardiac resynchronization therapy in clinical practice during exercise. Netherlands Heart Journal. 2013 Oct;21(10):458-63. doi: 10.1007/s12471-013-0438-3

B Oude Velthuis, MMD Molenaar, HG Reinhart Dorman, JY Stevenhagen, MF Scholten, J van der Palen, JM van Opstal. Use of three-dimensional computed tomography overlay for real-time cryoballoon ablation in atrial fibrillation reduces radiation dose and contrast dye. Netherlands Heart Journal. 2017 Jun; 25(6): 388393. doi: 10.1007/s12471-017-0962-

MMD Molenaar, CC Timmermans, T Hesselink, MF Scholten, RMA Ter Bekke, JGLM Luermans, M Brusse-Keizer, K Kraaier, B Ten Haken, JG Grandjean, K Vernooy, JM van Opstal. Shorter Cryoballoon Applications Times Do Effect Efficacy but Result in Less Phrenic Nerve Injury: Results of the Randomized 123 Study. Pacing and Clinical Electrophysiology. 2019 May; 42(5): 508-514. doi: 10.1111/pace.13626.

MMD Molenaar, T Hesselink, RMA Ter Bekke, MF Scholten, R Manusama, L. Pison, M Brusse-Keizer, K Kraaier, B Ten Haken, JG Grandjean, CC Timmermans, JM van Opstal. Shorter RSPV cryoapplications result in less phrenic nerve injury and similar 1-year freedom from atrial fibrillation. Accepted with revisions for publication in Pacing and Clinical Electrophysiology

MMD Molenaar, T. Hesselink, MF Scholten, K Kraaier, DE Bouman, M BrusseKeizer, YJ Stevenhagen, PHFM van Dessel, B ten Haken, JG Grandjean, JM van Opstal. High incidence of (ultra)low oesophageal temperatures during cryoballoon pulmonary vein isolation for atrial fibrillation. Accepted with revisions for publication in Netherlands Heart Journal 


\section{Mondelinge presentaties}

Optimization of cardiac resynchronization therapy in clinical practice during exercise. Venice Arrhythmias Venetie 2013

Less than two minutes, second generation cryoballoon applications achieves acute PVI in 79\% without phrenic nerve palsy: preliminary results of the 1-2-3 study. NVVC congres Noordwijkerhout 2015

Op glad ijs? Voorlopige resultaten van de 123-studie - Prospectieve studie naar de optimale duur van cryotherapie in de behandeling van atriumfibrilleren.

Wetenschapssymposium MST Enschede 2016

Op zoek naar de ideale vriestijd - Voorlopige resultaten van de 123-studie. Prospectieve studie naar de optimale duur van cryotherapie in de behandeling van atriumfibrilleren. DRES congres Nijkerk 2016

Less than two minutes cryoballoon applications result in comparable acute and 6months success for the treatment of atrial fibrillation but reduced incidence of phrenic nerve palsy: preliminary results of the 123-study. ESC congres Rome 2016

Acute Safety and Efficacy Outcomes of Shorter Cryoballoon Application Times for Cryoballoon Pulmonary Vein Isolation: Results of the 123-Study. NVVC congres Arnhem 2017

Acute Safety and Efficacy Outcomes of Shorter Cryoballoon Application Times for Cryoballoon Pulmonary Vein Isolation: Results of the 123-Study.

Wetenschapssymposium MST Enschede 2017 


\section{Poster presentaties}

Optimization of cardiac resynchronization therapy in clinical practice during exercise. NVVTG congres Utrecht 2013

High incidence of low esophageal temperatures during cryoballoon therapy for atrial fibrillation. NVVC congres Noordwijkerhout 2015

High incidence of low esophageal temperatures during cryoballoon therapy for atrial fibrillation. Heart Rhythm Boston 2015

Less than two minutes, second generation cryoballoon applications achieves acute PVI in 77\% without phrenic nerve palsy: preliminary results of the 1-2-3 study. EHRA Europace - Cardiostim Milaan 2015

High incidence of low esophageal temperatures during cryoballoon therapy for atrial fibrillation. EHRA Europace - Cardiostim Milaan 2015

Less than two minutes, second generation cryoballoon applications achieves acute PVI in $76 \%$ without phrenic nerve palsy: preliminary results of the 1-2-3 study. ESC congres Londen 2015

High incidence of low esophageal temperatures during cryoballoon therapy for atrial fibrillation. ESC congres Londen 2015

Prevalence and clinical consequences of collateral findings detected by computed tomography in patients with atrial fibrillation undergoing pulmonary vein isolation. Wetenschapssymposium MST Enschede 2016

Prevalence and consequences of collateral findings detected by computed tomography in patients undergoing pulmonary vein isolation for atrial fibrillation. Cardiostim - EHRA Europace Wenen 2017

High incidence of low oesophageal temperatures during 2nd generation cryoballoon therapy for atrial fibrillation: Predictive value of pulmonary veinoesophageal distance measured by CT scan. ESC congres Rome 2016 


\section{Dankwoord}

Dit dankwoord is mijn bel, na het startschot een kleine acht jaar geleden betekent de bel, zoals altijd: nog één ronde te gaan. Er zijn vele overeenkomsten te vinden tussen topsport en een promotietraject, heb ik de afgelopen jaren geleerd. Het vereist een enorme bak doorzettingsvermogen en er is zelden een lineair verband tussen je inspanning en de resultaten. Het is beiden zo simpel als "even vaak opstaan als vallen”. In tegenstelling tot een koers, waarin over het algemeen bekend is na hoeveel kilometer de finish ligt, bleef het eindpunt van deze koers, de promotiedatum, lange tijd een vraag. En zelfs nadat de finish neergelegd was, bleek de finish niet op de finishlijn te liggen. lets wat overigens zelfs in de grootste wielerkoers ter wereld kan gebeuren. Om een goede koers te rijden is het soms ook nodig om veel geduld te hebben en af te wachten tot het moment suprême. Komt die ervaring me nu eventjes mooi uit...

De laatste ronde voelt als een ere-rondje. Dank aan eenieder die eraan heeft bijgedragen, op welke manier dan ook, dat ik dit ere rondje mag rijden. Graag laat ik een aantal mensen specifiek meerijden.

Dr. van Opstal, Jurren, je staat in dit proefschrift vernoemd als co-promotor, maar de dagelijkse begeleiding en het richting geven aan mijn promotie kwam natuurlijk van jou. Je hebt me veel ruimte gegeven voor zelfstandigheid en eigen invulling. Degenen die mij kennen, zullen zeggen dat ik het wat dat betreft niet beter had kunnen treffen. Toen ik tegen het einde van mijn promotie aangaf je hulp ècht even nodig te hebben, was je er ook. Dank voor alles, ik denk dat we samen enorm trots kunnen zijn op de inhoud van dit proefschrift.

Prof. dr. ir. ten Haken, Bennie, aan het begin van mijn promotie was je nog geen professor. Tijdens je oratie heb ik veel onderdelen gezien waar ik een bijdrage aan heb mogen leveren. De gesprekken met jou waren vaak hoogover, wetenschappelijke vergezichten en altijd weer nieuwe ideeën over volgende stappen voor de vakgroep, practica, of aan te schaffen imaging apparatuur. Dit heeft mede mijn manier van (wetenschappelijk) denken gevormd en ik kijk er met plezier op terug. Dank daarvoor.

Prof. dr. Grandjean, Jan, aan het einde van mijn periode in Enschede heb ik jou bij mijn promotie betrokken. Wat ben ik blij dat je dit wilde doen. Als vader van dochters in dezelfde leeftijdscategorie als ik, nam je deze rol soms ook in onze samenwerking op je. Heel hartelijk dank voor je raad maar ook zeker je geruststelling en relativering. 
Jurren, Bennie, Jan, afgelopen jaren hebben we een unieke samenwerking gehad tussen UT en MST. Dit heeft mij ook in staat gesteld om vele plekken op de wereld te bezoeken voor congressen en lezingen. Dank voor deze hoogtepunten.

De leden van mijn promotiecommissie dank ik voor de bereidheid om in deze commissie plaats te nemen, en voor het zorgdragen voor alle taken die daaraan verbonden zijn. Speciale dank aan dr. Scholten. Marcoen, ik herinner me goed de eerste keer dat je de deur van een EFO-kamer voor me opende. Ik was nog student en gefascineerd door de grote hoeveelheid monitoren, signalen en beelden. Ik vroeg me direct, mezelf kennende ook hardop, af waarom men dit de afgelopen studiejaren voor mij verborgen had gehouden. Verder heb ik uiteraard mooie herinneringen aan alle uren die we samen op de fiets doorbrachten in binnen en buitenland. Deze halen het echter niet bij de onvergetelijke ducktour in Boston.

Alle co-auteurs van de artikelen in dit proefschrift bedank ik voor hun waardevolle bijdrage. Dr. Brusse-Keizer, Marjolein, bedankt dat ik na overleggen altijd gesteund door het "Marjolein-is-het-met-me-eens argument" terug kon keren naar de andere co-auteurs. De collega's uit het Maastricht UMC+ dank ik hartelijk voor hun aandeel in de 123-studie.

Alle cardiologen van het Medisch Spectrum Twente wil ik bedanken voor de samenwerking in de vijf jaar dat ik op deze afdeling heb doorgebracht. In meer of mindere mate heeft eenieder haar of zijn bijdrage geleverd aan dit proefschrift. Uiteraard speciale dank hierin aan de sectie electrofysiologie voor het in mij gelegde vertrouwen.

Dank ook aan alle patiënten voor jullie vertrouwen. Dank voor jullie deelname aan de studies, voor jullie bereidwilligheid om een bijdrage te leveren aan het verbeteren van de zorg voor anderen.

Dit proefschrift was ook niet mogelijk geweest zonder de inzet van de rest van de afdeling cardiologie. Het secretariaat, de echo en hartfunctieafdeling, en specifiek de pacemaker en electrofysiologie afdeling. Vele uren heb ik met jullie op de HCK doorgebracht en vele uren hebben ook jullie, met de stopwatch in de hand, besteed aan het uitvoeren van de studies. Wanneer er per abuis een seconde te vroeg of te laat op een knop werd gedrukt, kwam altijd de vraag "hoe ik dit nu ging verkopen in mijn proefschrift". Met als antwoord dat het toch mooi was dat er in mijn proefschrift "foutje Harry", "Bob lette niet op" of "Dennis zijn schuld” kwam te staan. Bij dezen. Dank allemaal voor de gezellige uren in het blauw achter het glas. 
Ik bedank specifiek de collega's waarmee ik een kamer gedeeld heb. Of het nu was op de oude HCK 4, op de verloskamer aan het Ariënsplein, in de CRE-ruimte na "de samensmelting” of op de UT. Bedankt voor jullie gezelligheid, jullie enthousiasme, dagelijkse babbels, de laatste nieuwtjes en de beste recepten. Voor jullie luisterend oor, droge humor, goeie tips en dresscode vrijdagen. Ik kon ze stuk voor stuk soms goed gebruiken en ze hebben mijn herinnering aan de promotietijd gevormd.

Mijn huidige werkgever DSW Zorgverzekeraar wil ik bedanken voor de mogelijkheid die ze mij geboden heeft om mijn werkzaamheden twee jaar lang te kunnen combineren met dit promotietraject.

Mijn promotietraject heb ik ook gecombineerd met een topsport carrière. De sport gaf me de fysieke uitlaatklep en het niveau gaf me de mogelijkheid om even iets totaal anders heel belangrijk te maken. Het bracht me ook veel nieuwe vrienden waar ik deze speciale tijd intensief mee heb mogen doorbrengen. Dank voor jullie afleiding, jullie fanatisme dat mij motiveerde om ook alles te geven, de uren en uren op de fiets waarin we het over van alles en ook over niks konden hebben. Inmiddels zijn we een paar jaar verder maar ik prijs me gelukkig dat ik jullie nog steeds mijn vrienden en vriendinnen kan noemen.

Datzelfde geldt uiteraard ook voor al mijn andere vrienden en vriendinnen, van het eerste uur, vanuit later in Enschede, en inmiddels ook vanuit Rotterdam. Dank voor jullie interesse, lieve en motiverende woorden, maar zeker ook voor alle gezelligheid en hilariteit die jullie me hebben gebracht en nog steeds brengen.

Een promotie met topsport combineren is geen sinecure, heb ik mezelf gedurende deze periode maar misschien nog wel meer na afloop ervan gerealiseerd. Het heeft me ontzettend veel gebracht maar er bleef weinig tijd over voor iets anders dan die twee. Naast mijn vrienden en vriendinnen hebben ook al mijn kennissen, familie en naasten dit gemerkt. Dank voor jullie begrip. Maar ook voor jullie onverminderde belangstelling in mij en mijn promotie. Dank ook voor het soms maar even niet meer vragen naar die promotie, wanneer dat voor de sfeer of mijn gemoedsrust beter was. Het is voor mij overduidelijk dat ik jullie deelgenoot wil maken van de voltooiing hiervan. Op dit moment is nog onduidelijk of ik jullie hier allemaal live bij aanwezig kan laten zijn. Ik hoop het van harte want dit feestje zou ik ontzettend graag met jullie allemaal vieren.

Heleen, Jeroen en de rest van het gezin + aanhang. Inmiddels voelen jullie voor mij ook als familie, met als basis het heerlijke huis in Haastrecht waar het regelmatig de zoete inval is. Dank voor jullie gastvrijheid, openheid, interesse en de vele 
wijntjes die we al gedronken hebben. Proost op nog vele die zullen volgen. Jeroen speciale dank voor je bijdrage aan dit proefschrift, fijn dat ik uitgebreid gebruik heb mogen maken van je vele jaren ervaring met promoveren.

Linnea, mijn paranimf, inmiddels al ruim twee jaar geen collega meer maar gelukkig nog steeds een dierbare vriendin. Je vrolijke gebabbel, je relativerende en nuchtere blik en je enorme energie om ergens iets van te maken hebben vele glimlachen op mijn gezicht getoverd. En: met al onze kleding-markt ervaring en jouw neusje voor styling weet ik zeker dat ik er, als de verdediging eenmaal daar is, fantastisch bij zal lopen.

Pap, mam, Joris en Liza, dank voor alles, veel daarvan is oneindig en altijd onvoorwaardelijk. Uiteraard ook mijn dank voor jullie bijdrage aan de totstandkoming van dit proefschrift, en het uit handen nemen van allerlei zaken. Vaak last minute, maar dat was nooit een probleem. Joris, je weet niet half hoe fijn het gevoel is dat ik mijn broer aan mijn zijde heb tijdens de verdediging. Pap, mam, ik hoor misschien nog wel dagelijks jullie woorden "als jij iets ècht wil, dan kun je het" in mijn hoofd terug. Jullie hebben mij het geloven in mezelf, mijn arbeidsethos en mijn enorme doorzettingsvermogen bijgebracht. Zonder dat alles had dit proefschrift er niet gelegen. Ik weet dat jullie enorm trots zijn, maar net zo trots waren geweest als ik de handdoek in de ring had gegooid. Ik heb het voor mezelf gedaan, deze marathon finishen, maar prijs me de gelukkigste dochter die er is, dat ik jullie nog steeds trots kan en mag maken.

Lieve Sjoerd, we zijn er. Laatst zei ik je nog, gekscherend, dat het met jou vaak maandagavond is; met mij is het ook zeer regelmatig maandagochtend geweest. Wat ben ik gelukkig dat jij er ook dan voor me bent. Met jou zijn voelt als thuiskomen. Ik kan niet wachten om het beeldscherm dicht te klappen en de omlijsting van dit proefschrift samen weer in het echt te gaan bekijken. Ik weet dat we samen bergen kunnen bedwingen en ook verzetten, ik geniet van het uitzicht dat zich voor ons uitstrekt. 


\section{Curriculum Vitae}

Marleen Maria Dirkje Molenaar werd op 15 februari 1987 geboren te Purmerend en groeide op in Monnickendam. Na het cum-laude behalen van haar WW-diploma aan het Bernard Nieuwentijt College Damstede te Amsterdam in 2005 verhuisde zij naar Enschede waar zij startte met de studie Technische Geneeskunde aan de Universiteit Twente. In 2012 behaalde zij haar master titel in de Technische Geneeskunde (specialisatie Medische Signalen) met een afstudeerscriptie over optimalisatie van cardiale resynchronisatie therapie tijdens inspanning. Daarna startte zij als promovenda en technisch geneeskundige in een gecombineerde aanstelling van zowel het Thoraxcentrum Twente, onderdeel van het Medisch Spectrum Twente, als de vakgroep Magnetic Detection \& Imaging behorend tot de faculteit Technische Natuur Wetenschappen van de Universiteit Twente. Van 2012 tot en met 2017 heeft zij haar promotietraject gecombineerd met een topsportcarrière in het marathonschaatsen. In 2018 verhuisde ze naar Rotterdam waar zij momenteel woonachtig is. Zij startte daar met haar huidige aanstelling als business analist op de functionele IT-afdeling van DSW Zorgverzekeraar, waar zij momenteel naast business analist ook de rol van product owner vervult. 